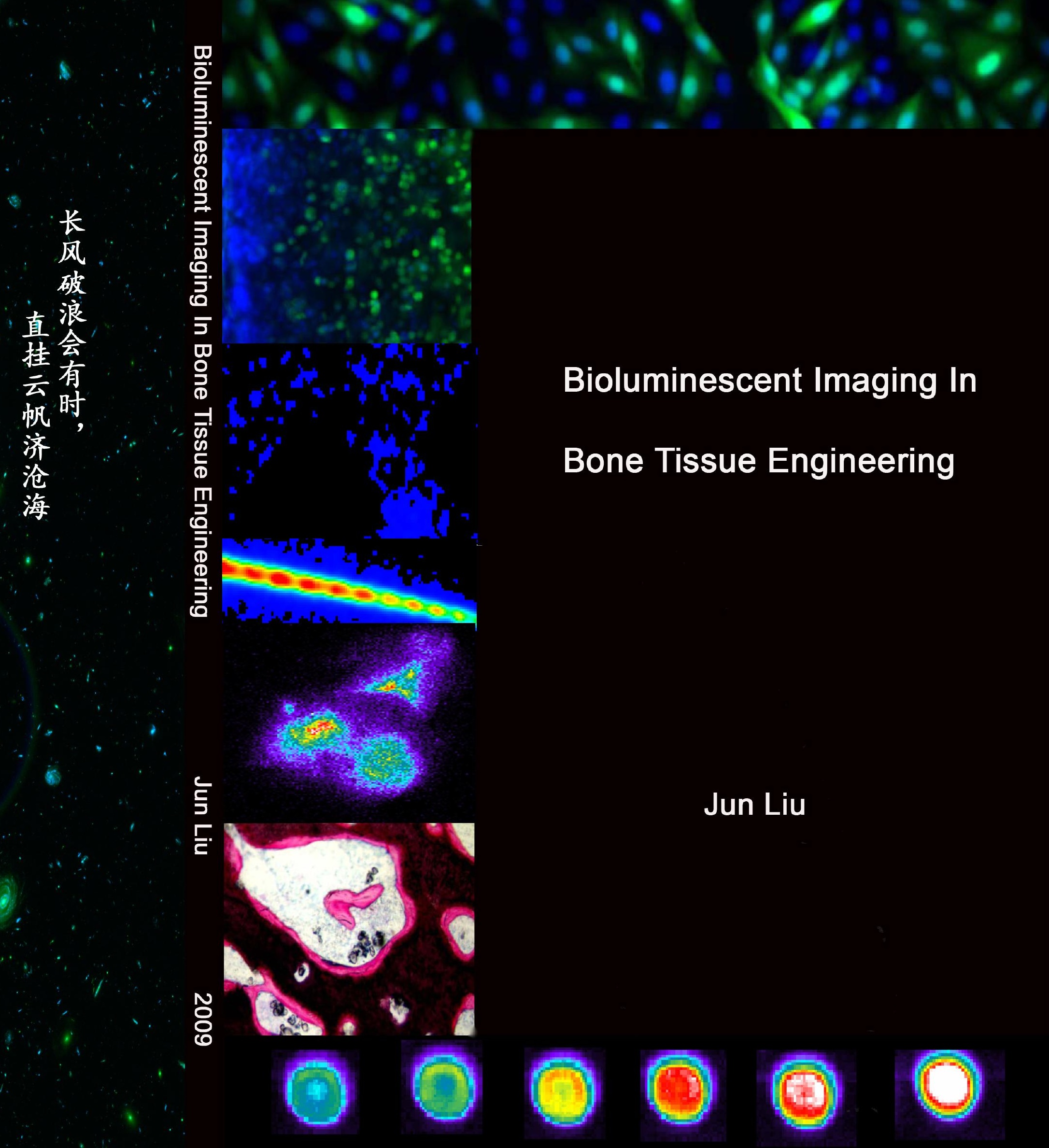




\title{
Bioluminescent Imaging in Bone Tissue Engineering
}

\author{
Jun Liu
}

2009 
Members of the Committee:

Prof. dr. G. van der Steenhoven

Chairman (University of Twente)

Prof. dr. C.A. van Blitterswijk Promoter (University of Twente)

Dr. J. de Boer

Co-Promoter (University of Twente)

Prof. dr. I. Martin

(University Hospital Basel, Switzerland)

Prof. dr. C. W.G.M. Lowik (Leiden University Medical Center)

Prof. dr. P. Buma

(Radboud University Nijmegen Medical Centre)

Dr. R. Arends

(Strategic Program Development)

Prof. dr. D.W. Grijpma

(University of Twente)

Prof. dr. V. Subramanian

(University of Twente)

\section{Jun Liu}

\section{Bioluminescent Imaging in Bone Tissue Engineering}

PhD Thesis, University of Twente, Enschede, The Netherlands

Copyright: J. Liu, Enscehde, The Netherlands, 2009. Neither this book nor its parts may be reproduced without written permission of the author.

\section{ISBN: 978-90-365-2908-2}

The research described in this thesis was financially supported by a research grant from Senter/Novem. The publication of this thesis was sponsored by

\section{סS Schering-Plough}

\section{biospace $1 a b$}

\section{Roche}

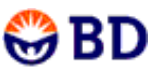

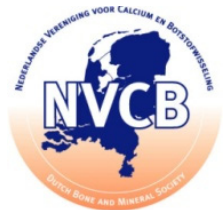

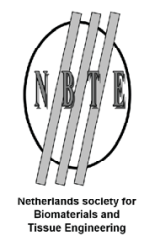

Printed by: Wöhrman Print Service, Zutphen, The Netherlands.

Cover design: Jun Liu.

The front cover shows some fluorescent and bioluminescent images taken by the author. The background of the back cover shows the universe space. 


\title{
BIOLUMINESCENT IMAGING IN BONE TISSUE ENGINEERING
}

\author{
DISSERTATION
}

\author{
to obtain \\ the doctor's degree at University of Twente, \\ on the authority of the rector magnificus, \\ prof. dr. H. Brinksma \\ on account of the decision of the graduation committee, \\ to be publicly defended \\ on Thursday $22^{\text {nd }}$ October 2009 at 13:15
}

by

Jun Liu

born on January $19^{\text {th }} 1981$

in Linfen, Shanxi Province, China 
献给我的父母
To my parents 


\section{Contents}

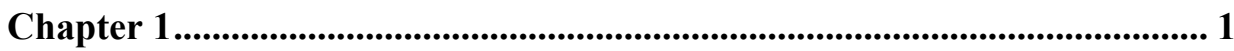

General introduction and aims

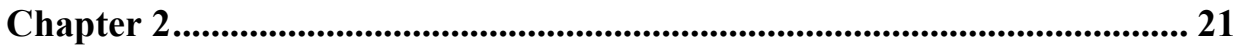

The effect of PKC activation and inhibition on osteogenic differentiation of hMSCs

Chapter 3

In vitro and in vivo bioluminescent imaging of hypoxia in tissue engineered grafts

Chapter 4

Imaging nutrient diffusion in tissue engineered grafts

Chapter 5 73

Non-invasive imaging of luciferase transgenic mice in bone tissue engineering

Chapter 6 .

General conclusion, discussion and future perspective

Summary

内容简介 109

Samenvatting 111

REFERENCE 115

Acknowledgements

Curriculum Vitae 



\section{Chapter 1}

\section{General introduction and aims}

Application of bioluminescent imaging for cell-based bone tissue engineering 


\section{Bone and Bone Tissue Engineering}

\section{Bone remodelling}

Bone, a specialized connective tissue, plays an important role as mechanical support, as protection of organs and in metabolic homeostasis of ions in the body. Bone is constituted by many different types of cells and by an extracellular matrix (ECM) which has the unique property to be calcified. The ECM is composed of collagen fibers (of which $90 \%$ collagen type I), oriented in a preferential direction, and noncollagenous proteins. Anatomically, there are two types of bone in the skeleton: flat bone (skull bones, scapula, mandible and ileum) and long bones (tibia, femur, humerus), which are formed by two mechanisms, intramem-branous and endochondral bone formation. The former process contributes mainly to the flat bones, while the long bones develop by both mechanisms. During intramembranous ossification, mesenchymal stem cells differentiate under the influence of local growth factors (e.g. fibroblastic growth factors (FGFs), bone morphogenic proteins (BMPs), Hedgehog, Parathyroid hormone related peptide (PTHrP)) and the transcription factors Runx2 and Osterix into osteoblasts, which deposit irregular woven bone. Next, woven bone is remodelled by osteoclasts and osteoblasts and replaced by mature lamellar bone. In endochondral ossification, the process starts with condensing mesenchymal cells which differentiate into chondrocytes, which will hypertrophise and mineralise. Blood vessels invade the calcified matrix and osteoblasts deposit bone.

Bone is not a static tissue but rather a dynamic one that undergoes modelling and remodelling throughout life. This process involves two main cell types, osteoblasts for bone formation and osteoclasts for bone resorption (Fig. 1). These two type cells originate from mesenchymal and haematopoietic stem cells respectively. Due to this property, bone is one of the few tissues that can self-repair without leaving a scar. The coordinated actions start at the trabecular bone surface in cancellous bone and Haversian systems in cortical bone. The remodelling time differs in cancellous and cortical bone and it generally takes around 3-4 months. All bone diseases are superimposed on this normal cellular remodelling process. Most widely known, the progressive bone loss due to aging in all humans begins at around 35 years of age and is due to the imbalance of bone resorption and formation. Skeletal loss caused by traumas, tumor resection or wear of implants is often too extensive for self-healing, and a bone substitute is needed to restore the skeletal function. 


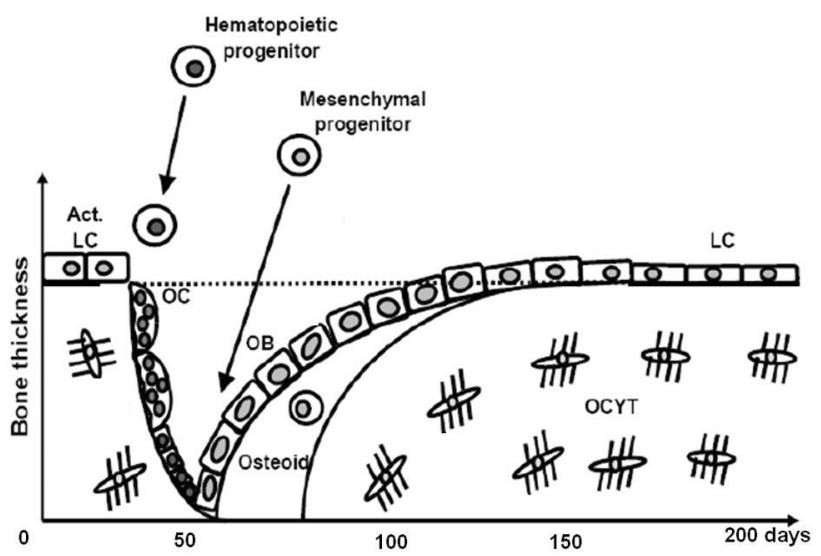

Figure. 1 Schematic model of time dependent bone remodeling. OB, osteoblasts; OC, osteoclasts; OCYT, osteocyte; LC, lining cells; Act. LC, active lining cells. (Adapted from Bone Histomorphetry book; Eriksen EF, et.al; 1994; Raven Press, New York, NY, USA.).

\section{Bone tissue engineering (BTE)}

As reported from the World Health Authority, every year there are over 500,000 bone grafts performed in the United States, to heal bone defects caused by trauma, diseases like cancer and age-related osteoporosis and fracture.This urges the improvement of current therapies on bone substitution in spinal fusion, fracture healing and bone defect reconstruction. Current strategies for bone grafting are listed in Table 1. Within the last century, bone grafting has seen a development from autologous bone grafting, allograft bone, demineralised bone matrix, calcium phosphate-based biomaterials to the administration of recombinant growth factors. The ideal bone graft substitute is still not available. An alternative strategy is to combine osteogenic cells with a scaffold material, referred to as bone tissue engineering.

\section{Key factors in BTE}

The procedure of cell-based bone tissue engineering is depicted in Fig. 2. Stem cells or progenitors are isolated from the patient and expanded in vitro to produce a sufficient number of cells, are then exposed to growth factors to commit the cell into the osteogenic linage, are seeded onto scaffolds, and implanted back into the patient [2]. Fig. 3 describes the process of bone formation on a TE graft in vivo [3]. Osteogenic stem cells or progenitors (green) 
Table 1. Bone graft substitutes: past, presence and future (modified from ref. [4]).

\begin{tabular}{|c|c|}
\hline & Description, Advantages and Drawbacks \\
\hline Autograft & $\begin{array}{l}\text { - } \text { Bone harvested from host donor sites. } \\
\text { - } \quad \text { Gold standard graft. } \\
\text { Donor site morbidity, inadequate amount, and inappropriate form and associated chronic donor site pain. }\end{array}$ \\
\hline Allograft & $\begin{array}{l}\text { - } \quad \text { Bone harvested from guest donor. } \\
\text { - } \quad \text { Attractive sources. } \\
\text { processing, increased cost, and non-availability world-wide due to financial and religious concerns. }\end{array}$ \\
\hline \multicolumn{2}{|c|}{$\begin{array}{l}\text { Osteoconduction: provide a passive porous scaffold to support or direct bone formation. Some research indicates that the } \\
\text { requisite pore size for bone ingrowth into porous implants is } 100 \text { to } 500 \mu \mathrm{m} \text {, and the interconnections must be larger than } 100 \\
\mu \mathrm{m} \text {. }\end{array}$} \\
\hline $\begin{array}{l}\text { Calcium } \\
\text { sulphate }\end{array}$ & $\begin{array}{l}\text { - A bone void filler, and the use of antibiotic-laden plaster in the treatment of infected bony defects. } \\
\text { OsteoSet (Wright Medical Technology, Arlington, TN), which was approved by FDA in 1996. It can be used } \\
\text { in presence of infection and it is comparatively cheap } \\
\text { - Three cases of inflammatory response and a single case of allergic reaction have been reported with the use of } \\
\text { this compound. }\end{array}$ \\
\hline $\begin{array}{l}\text { Porous } \\
\text { coralline } \\
\text { ceramics }\end{array}$ & $\begin{array}{l}\text { - Corals made by marine invertebrates have skeletons with a structure similar to both cortical and cancellous } \\
\text { bone, with interconnecting porosity. } \\
\text { One approach is to use coral directly in calcium carbonate form. These materials are called natural corals. The } \\
\text { trade name for natural coral is Biocoral (Inoteb, Saint-Gonnery, France). The other process is Replamineform } \\
\text { process that converts calcium carbonate to hydroxyapatite. }\end{array}$ \\
\hline Metal & $\begin{array}{ll}- & \text { Titanium and titanium alloys. } \\
\text { - } & \text { Good mechanical properties for load bearing sites; good corrosion resistance. } \\
\text { - } & \text { Bionert for bone and surrounding tissue growth. }\end{array}$ \\
\hline
\end{tabular}




\begin{tabular}{|c|c|}
\hline $\begin{array}{l}\text { Calcium } \\
\text { phosphate } \\
\text { ceramics and } \\
\text { cements }\end{array}$ & $\begin{array}{l}\text { - Hydroxyapatite (HA), tricalcium phosphate (TCP), biphasic calcium phosphate (BCP). } \\
\text { - Bioactive and biocompatible for tissue bonding and bone ingrowth; some even have the osteoinductivity at } \\
\text { ectopic sites. } \\
\text { - } \quad \text { Limited mechanical strength, not mouldable intraoperatively and also poor fatigue characteristics. }\end{array}$ \\
\hline $\begin{array}{l}\text { Synthetic } \\
\text { polymers }\end{array}$ & $\begin{array}{l}\text { - Collagen, hydrogel, chitosan, and multi-types of polymers. } \\
\text { - } \quad \text { Biocompatible, degradable, being easily tailored to combine with growth factors. } \\
\text { - } \quad \text { Poor mechanical strength, easy to get worn and biodegradable. }\end{array}$ \\
\hline \multicolumn{2}{|r|}{ Osteoinduction: induce differentiation of stem cells into osteogenic cells. } \\
\hline DBM & $\begin{array}{l}\text { - Initial studies performed by Urist in 1960. It includes the non-collagenous proteins, bone osteoinductive growth } \\
\text { factors, the most significant of which is BMP and type I collagen. } \\
\text { - Osteoinductive capacity in human and animals. } \\
\text { - Poor mechanical property, difficult to handle. }\end{array}$ \\
\hline $\begin{array}{l}\text { Growth } \\
\text { factors: }\end{array}$ & $\begin{array}{l}\text { - Transforming growth factor (TGF- } \beta \text { ), bone morphogenetic proteins (BMPs), fibroblast growth factors (FGFs), } \\
\text { insulin-like growth factors (IGFs), and platelet-derived growth factors (PDGFs). Most were approved in animal } \\
\text { studies, and some are used in clinical therapy. } \\
\text { - } \quad \text { The need to find out the proper delivery methods, and high cost. }\end{array}$ \\
\hline \multicolumn{2}{|r|}{ Osteogenesis: provide stem cells with osteogenic potential, which directly lay down new bone. } \\
\hline $\begin{array}{l}\text { Bone } \\
\text { marrow } \\
\text { aspirate }\end{array}$ & $\begin{array}{l}\text { - Bone marrow has been used to stimulate bone formation in skeletal defects and nonunions. } \\
\text { - Being performed percutaneously, without almost any patient morbidity. } \\
\text { - Limited number of stem cells: approximately one of every } 100,000 \text { nucleated cells aspirated from bone marrow } \\
\text { is a stem cell. }\end{array}$ \\
\hline \multicolumn{2}{|r|}{ Combined: provide more than one of the above mentioned properties. } \\
\hline Composites & $\begin{array}{l}\text { - Tissue engineering. } \\
\text { - These constructs are likely to encompass additional families of growth factors, evolving biological scaffolds, } \\
\text { and incorporation of mesenchymal stem cells. }\end{array}$ \\
\hline
\end{tabular}




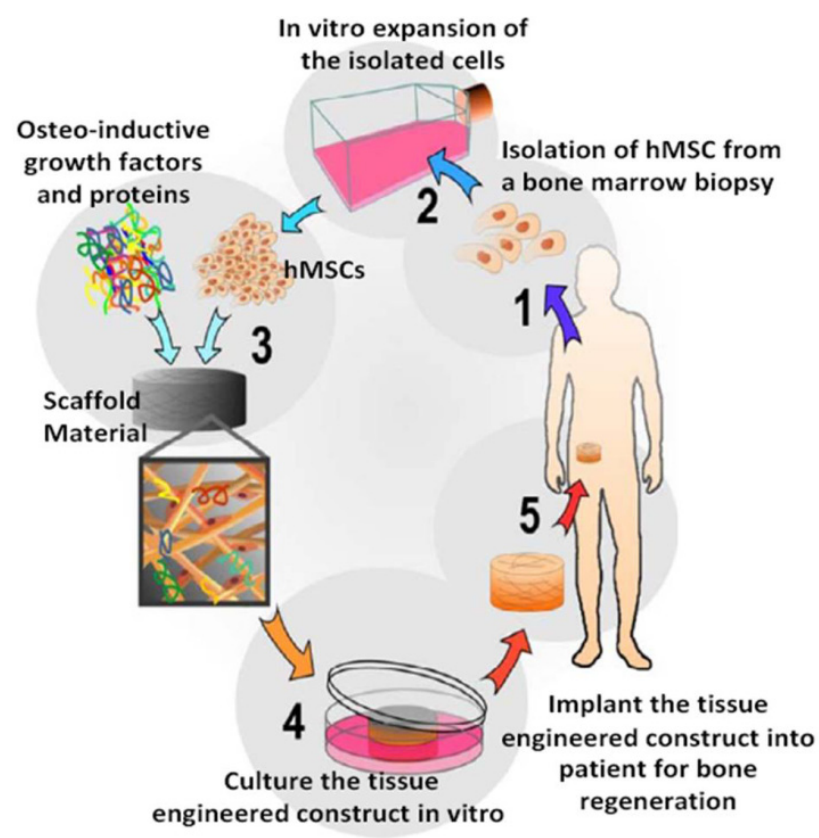

Figure. 2 The concept of cell-based bone tissue engineering. (Adapted from Tissue Engineering book; Prof. Clemens van Blitterswijk, et.al; 2007; ISBN: 978-0-12-370869-4; ELSEVIER.)

A

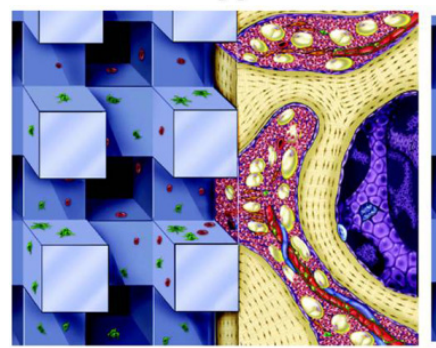

B

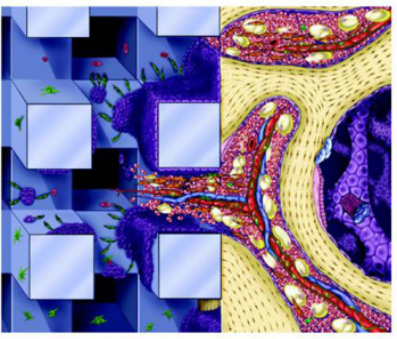

C

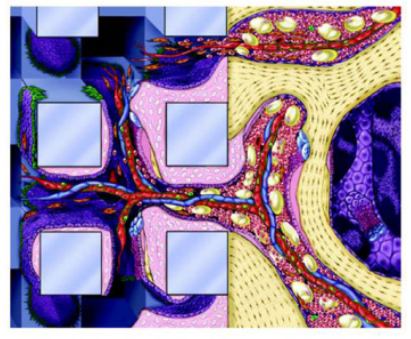

Figure. 3 Illustration of the process of bone ingrowth into a tissue engineering construct with a pore size of $\sim 150 \mu \mathrm{m}$. (Adapted from ref. [3])

adhere and distribute evenly in the scaffold with a pore size of $150 \mu \mathrm{m}$. Other non-osteogenic cells (pink) may also adhere to the matrix (Fig. 3A). Several days later, host cells migrate into the matrix, including inflammatory cells, progenitor cells from local stem cell sources and vascular endothelial cells which support angiogenesis (Fig. 3B). Following, woven bone is initially formed and replaced by lamellar bone, forming an interconnected network extending deeper within the implant. Meanwhile, void spaces within the scaffold are remodelled into bone marrow elements (Fig. 3C). Cells in deeper 
regions of the graft may not survive due to the lack of nutrients such as oxygen, glucose and others. Evidently, BTE is a multi-disciplinary field of science, including biology, chemistry, physics and engineering. A wealth of research has provided proof of concept in animal models, and BTE has also been tested in clinical trials [5-7]. Warnke reported a clinical case where a titanium mesh seeded with bone marrow and recombinant human bone morphogenetic protein 7 was used to reconstruct a mandibular defect [8]. Meijer et al. reported on a jaw defect clinical study in which differentiated bone marrow stem cells were seeded onto ceramic particles. 1 out of 6 patients convincingly formed new bone induced by the implanted human mesenchymal stem cells (hMSCs) [9]. Despite these studies, clinical application is still limited due to the relatively limited amount of bone formed [10,11]. As such, there is a great need to optimize each step to improve the power of BTE. Knowledge about osteogenic differentiation of hMSCs is a pre-requisite to achieve this.

\section{Mesenchymal stem cells}

Mesenchymal stem cells (MSCs) or stromal cells derived from bone marrow are a group of cells contributing to skeletal tissues. They were identified by Friedenstein and coworkers as a minor subpopulation of bone marrow cells (see ref [12] for a review). Their frequency in bone marrow is low, around $0.01 \%$ to $0.001 \%[13,14]$. MSCs are characterized by their fibroblastlike morphology, colony forming unit, and multipotency [15]. MSCs can be induced to differentiate into the osteogenic, adipogenic, chondrogenic and many other lineages including myoblasts in vitro and in vivo (Fig. 4A) [16, 17]. Later, MSCs were isolated from many other types of tissues including adipose tissue, tibia, femur, lumbar spine, trabecular bone and placenta [18-20]. Advantages of using MSCs in BTE are the facts that they are not very sensitive to immunogenic rejection, they do not display tumor formation and there are no ethical issues associated with them.

The term 'mesenchymal' refers to the mesenchymal germ layer during embryogenesis, which later constitutes the connective tissues. MSCs can be isolated readily by virtue of their adherence to tissue culture plastic, and are cultured with fetal bovine serum. MSCs can not be recognized and selected by a single marker. Instead, they express a complex pattern of molecules including STRO-1, CD105, CD54, CD13, CD106, CD90 and CD29, but not the hematopoietic markers CD45 and CD34 [16, 21, 22]. Cell size and morphology vary among different MSC colonies, and the same is true for growth rate and 
differentiation capacity, which is also donor-dependent both in vitro and in vivo [23]. For instance, the levels of bone alkaline phosphatise (ALP) of MSCs, an early marker for osteogenic differentiation, differ largely between different donors, varying from $1 \%$ to $33 \%$. Furthermore, the amount of in vivo bone formation induced by dexamethasone (Dex) treatment varies tremendously. To bridge a large bone defect, large numbers of differentiated MSCs are needed. Due to its naturally rare occurrence, in vitro expansion in tissue culture flasks is required, even though it is known to compromise the differentiation capacity of hMSCs.
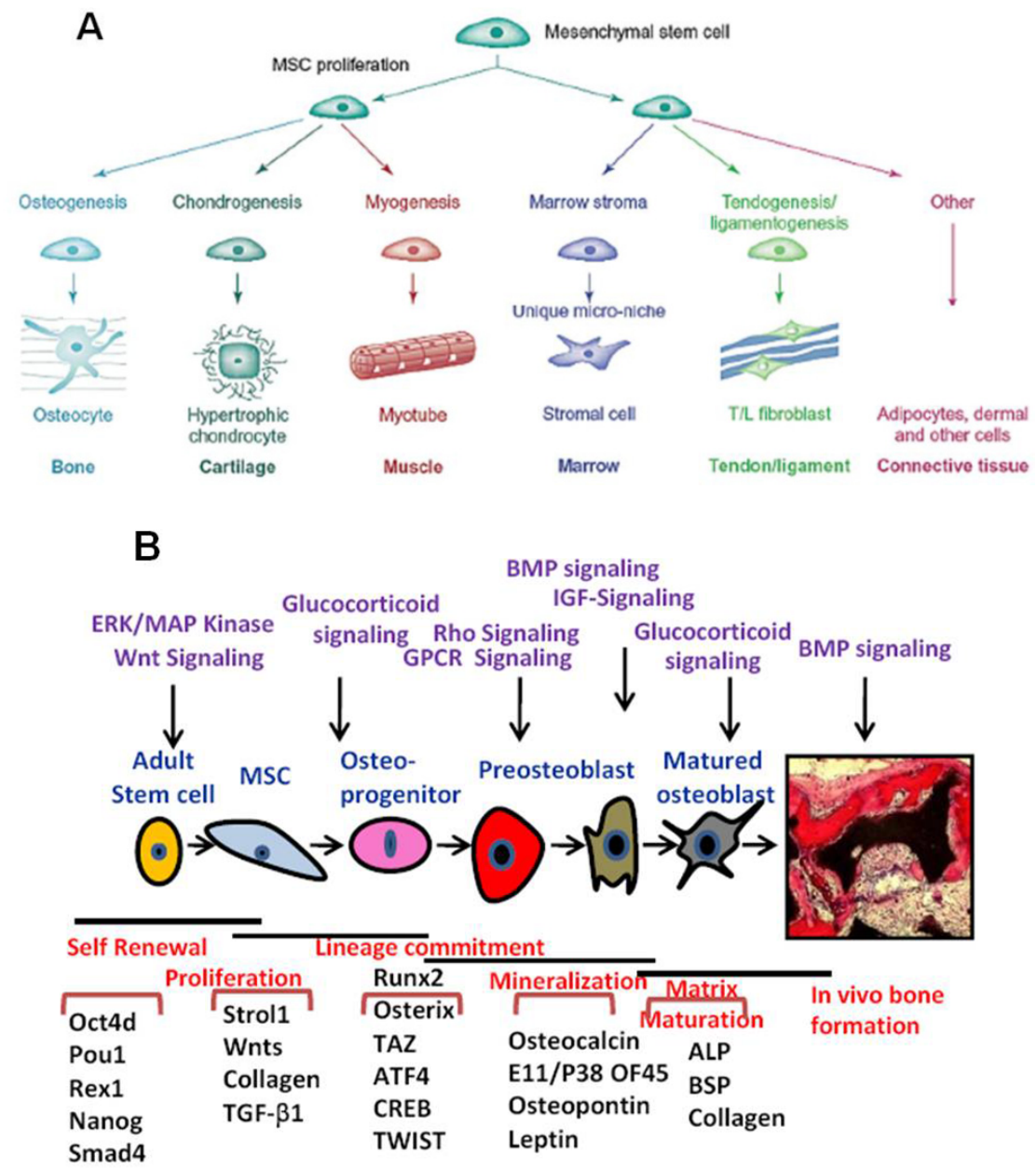

Figure. 4 (A) Schematic depiction of mesenchymal stem cell multipotency. (Adapted from ref. [17].). (B) Molecular and genetic cues impinging on the proliferation or differentiation of hMSCs. (Adapted from the PhD thesis of Dr. Siddappa, 2007, ISBN: 978-90-365-2583-1; The Netherlands.) 
Understanding the biology of hMSCs is crucial to improve hMSCs proliferation, differentiation and bone formation. Some growth factors are known to stimulate hMSC proliferation including platelet-derived growth factor (PDGF) [24], epidermal growth factor (EGF) [25] and basic fibroblastic growth factor (bFGF) [26]. We and others reported that canonical Wnt signalling stimulates hMSCs in vitro proliferation without influencing osteogenesis [2729]. As for osteogenic differentiation, the orchestrated effects of multiple different proteins, cytokines and transcription factors temporally regulate the process as depicted in Fig. 4B. Cbfa-1 is a master regulator of osteoblastogenesis, demonstrated by the fact that Cbfa- 1 knockout mice exhibit impaired chondrogenic and osteogenic differentiation, resulting in postnatal death due to skeletal failure [30,31]. Osteoblasts synthesize an extracellular matrix of around $90 \%$ collagen type 1 and $10 \%$ non-collagenous proteins. Bone mineral deposition occurs on the collagen matrix, and the mineral crystals aggregate further. Next, osteoblasts terminally differentiate into osteocytes which are located in lacunae and have metabolic functions. Interfering in osteocyte maturation leads to retarded skeletal ossification [32]. In our laboratory, we are exploiting molecular pathways to improve the current hMSCs culture protocols and to improve their bone formation capacity both in vitro and in vivo. For instance, activation of the protein kinase A pathway using cyclic AMP was found to drastically enhance bone formation by hMSCs [28].

It is not sufficient to focus on pharmaceutical/genetic treatment of hMSCs in 2 dimensional, because in nature and in BTE applications, cells are grown in a 3-dimensional matrix and biomaterials. Thus, it is important to investigate biomaterial properties and its interaction with cells.

\section{Biomaterials and scaffolds}

The biomaterials used in bone tissue engineering are classified and shown in Table 1. Besides serving as cell carriers and providing mechanical support, the materials may play an active role in defining cell fate [33]. Basic requirements of biomaterials for BTE are biocompati-bility, proper mechanical properties, a certain pore size and porosity, architecture and biodegradability. The materials should allow tissue and blood vessel infiltration and growth, and should be optimal for nutrient exchange. Porosity indicates the ratio of the interconnected pore volume. The porosity and pore size influence the survival of the grafted and host cells, and determine tissue ingrowth. Recently, research interest in the architecture of biomaterials has shifted from macro- (bigger than 
$50 \mu \mathrm{m})$ to micro- (less than $50 \mu \mathrm{m}$ ) and even to nano-scale. For instance, it has been reported that micro-porous biphasic calcium phosphates (BCP) sintered at $1150^{\circ} \mathrm{C}$ will induce ectopic bone formation in vivo whereas macro-porous $\mathrm{BCP}$ sintered at $1300^{\circ} \mathrm{C}$ does not [34]. In addition, several reports describe the effect of nano-scale topography on differentiation of hMSCs [35]. These studies stimulate material experts in developing the next generation of biomaterials with tailored surface pore sizes and patterns. However, protein and growth factor treatment is still the chosen method to direct cell proliferation and differentiation.

\section{Nutrition}

The growth of hMSCs on porous biomaterials poses the next challenge for tissue engineers: nutrient supply. In nature, cells receive nutrients by diffusion from blood vessels within $100-200 \mu \mathrm{m}$. Nutrient deficiency occurs to cells located too far away from blood vessels, as seen in the occurrence of cardiac ischemia. Nutrient limitation is a classical issue in the field of tissue and organ transplantation. It received wide attention of scientists and clinicians following the work of Folkman on the "dormant diameter" of in vitro tumor cultures [36]. Folkman suggested that tumors could not exceed a critical size, without connection to the vascular system, due to limited diffusion. Actually, in 1961, Greene demonstrated this point by showing that the implanted tumor regrew after being switched into a richer environment. Following these studies, more and more studies revealed that nutritional problems are not limited to tumors, but play a role in the transplantation of many tissues and organs. Physiological studies show that capillaries are spaced by a maximum of $200 \mu \mathrm{m}$, which suggests the range of nutrient diffusion in vivo [37].

Nutrient limitations also apply to cell-based tissue engineering. When the task in this field moves "from lab bench to the clinic", a new challenge appears due to the size of the engineered tissue. There are already several tissue engineered products applied clinically, such as skin, cartilage, and bladder, which are either thin layered structures (skin, bladder) or avascular (cartilage) [38-40]. Other tissues, such as bone, muscle, myocardium etc, are not yet clinically applied. The architecture of these tissues exceeds $200 \mu \mathrm{m}$ and the tissues are therefore heavily vascularised. To engineer those tissues, it is generally accepted that nutrient diffusion is not sufficient to support cell survival in the centre of the graft, both in vitro and in vivo. During conventional in vitro static culturing of a $3 \mathrm{D}$ cell-scaffold composite, nutrition is delivered 
merely via diffusion with a limitation of $200 \mu \mathrm{m}$. Consequently, cell survival or tissue formation is localized to the periphery of scaffolds, whereas the central part has limited tissue formation or even necrosis [41, 42]. When cells are implanted in vivo, nutrition support comes from the adjacent host blood vessels. For big grafts (more than several hundreds of micrometers thickness), the constructs need their own blood vessels and capillaries to provide nutrition to and waste transport to and from the tissue. After implantation, blood vessels from the host will invade the implant. However, it takes days to develop the new vasculature network in the graft from host blood vessels [43], which means that during this reconstructive period, the cells far from the host capillaries experience nutrient limitations, which can result in cell death (Fig. 5). When the graft has been vascularized, the cells that survived can proliferate and expand again. Therefore, provision of sufficient nutrient both in vitro and in vivo is an important issue in tissue engineering.

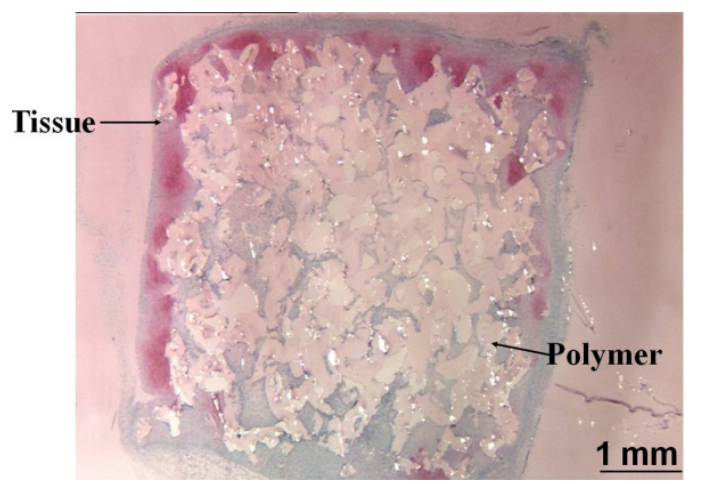

Figure. 5 Heterogeneous tissue distribution in a chondrocyte-polymer graft after 3-week implantation. After explantation, safranin-O stained the glycosaminoglycans in the cartilage ECM the pink. Note that no tissue formation was detected in the inner part of scaffold, which is probably due to cell death. (Adapted from the $\mathrm{PhD}$ thesis of Dr. Malda, 2003, ISBN: 90-365-1959-4; The Netherlands.)

Among the nutrients essential for cell survival, oxygen seems to be particularly important, due to its high consumption rate, low solubility in culture medium and relatively slow diffusion rate [44]. It was reported that an $\mathrm{O}_{2}$ gradient or limitation relates to cellular proliferation and extracellular matrix production $[41,45,46]$. When supplied with enough $\mathrm{O}_{2}$, cells depend on aerobic glycolysis for the energy supply, while under hypoxia glycolysis is largely anaerobic where more lactate would be produced. The lack of removal of waste products, in turn, is an additional threat to cells, hampering protein synthesis and cell survival.

Thus, it is still a challenge to grow large grafts both in vitro and in vivo. Current strategies to improve nutrient availability include improving diffusion by controlling pore size and porosity of scaffolds, by using flow perfusion bioreactors, and by stimulating vascular ingrowth using growth factor release 
(e.g. vascular endothelial growth factor (VEGF) and bFGF) and approaches to pre-vascularize the grafts [47]. For instance, it has been reported that ingrowth of vessels is faster in scaffolds with pores of $250 \mu \mathrm{m}$ than in scaffolds with smaller pores [48]. Similarly, the convective flow of perfusion bioreactors enhances the transport of oxygen and nutrients to the interior of the construct beyond the limits of diffusion, leading to improved cell viability in the interior and more homogeneous cell distribution in the scaffold [49-51]. The slow release of growth factors such as VEGF and bFGF promote angiogenesis through the sprouting of local vessels $[52,53]$. It was reported that coimplantation of VEGF and BMP2 induced more in vivo bone formation than BMP2 alone [54]. Levenberg et al. demonstrated that a prevascular network of endothelial cells improved skeletal muscle tissue survival after implantation in vivo [55]. Recently, Hung et al. demonstrated that short-time hypoxia $\left(1 \% \mathrm{O}_{2}\right)$ treatment of hMSCs enhanced their engraftment in vivo, suggesting that shortterm exposure to hypoxia before transplantation might be a simple way to improve the transplant survival [56]. This is probably because hypoxia activates VEGF gene transcription and stabilization [57-59].

\section{Molecular imaging of BTE}

To investigate bone remodelling and formation with the final goal to be applied in the clinic, animal models are required to represent the in vivo environment. In our lab, we employ immune-deficient mice as a model to investigate ectopic bone formation induced by hMSCs and human or mouse embryonic stem cells $[28,60]$. Moreover, transgenic small animals are widely used to investigate gene function in tissue homeostasis. The most important technique to assess the success of a tissue engineered grafts is post-mortem histology by tissue staining or enzymatic assays. Because this represents an endpoint analysis and large variation occurs among individual animals, a large number of animals are required to obtain temporal and statistically significant results. During the last decade, a lot of progress has been achieved on adopting new techniques for in vivo imaging of small animals, including clinically applied technologies such as ultrasound imaging, single photon computed tomography (SPECT), positron emission tomography (PET), magnetic resonance imaging (MRI) and X-ray computed tomography (CT) (Table 2) [61, 62]. These imaging modalities offer scientists the spatial and temporal information in a faster and convenient manner. For instance, MRI has been used 
to detect ectopic bone formation in rats [63]. Furthermore, $\mu \mathrm{CT}$ is a very efficient and direct tool to monitor bone morphology in disease [64]. Due to differential scatter and absorption of X-rays in tissue, $\mu \mathrm{CT}$ can reconstruct a bone image and offer quantitative data on bone density. However, most of these techniques rely on the spatial and anatomical derangements from normal patterns, thus omitting the intrinsic genetic and molecular information. A second drawback of $\mu \mathrm{CT}$ is that radiation exposure limits the frequency of use. Molecular imaging is possible by SPECT and PET, techniques which use radioactive isotopes such as the gamma-emitters technetium- $99 \mathrm{~m}\left({ }^{99 \mathrm{~m}} \mathrm{Tc}\right)$, indium-111 ( $\left.{ }^{111} \mathrm{In}\right)$ and the positron emitter fluorine-18 $\left({ }^{18} \mathrm{~F}\right)$. In the clinic, ${ }^{18} \mathrm{~F} / \mathrm{PET}$ was initially used to quantify bone formation in the 1990 s because F-18 is taken up by mineralizing bone in direct correlation to osteoblastic activity [65]. Today, more and more research on ${ }^{18} \mathrm{~F} / \mathrm{PET}$ and other labelled isotopes is reported to detect skeletal metastases or lesions in Paget's disease and prostate and breast cancers which induced increased bone turnover [66, 67]. ${ }^{18} \mathrm{~F}$-Fluoride uptake was increased in malignant bone, which reflected the increased regional blood flow and bone turnover characterizing the lesions. SPECT/PET together with $\mathrm{CT} / \mathrm{MRI}$ is used to clinically diagnose early bone metastases.

\section{Optical molecular imaging in animals and cells}

Optical imaging of living cells and animals has grown into an important tool in biomedical research both in vitro and in vivo [68]. It employs a reporter gene under the control of a regulatory sequence of interest. As such, the techniques is particular suitable for pre-clinical research in rodents. Both fluorescence and bioluminescence can reconstruct in vivo 3D images by detecting light intensity at different angels [69].

Fluorescence dyes have been used for decades in cell biology to detect molecular localisation, e.g. DAPI and phalloidin to detect DNA and actin respectively. The discovery of green fluorescent protein from the jellyfish Aequoria victoria made it possible to make fluorescent chimeric proteins (GFP) and to design fluorescent reporter assays by placing GFP under the control of a promoter of interest [70]. With the discovery of more fluorescent proteins, such as yellow fluorescent protein (YFP) and cyan fluorescent protein (CFP), single cells can be labelled with multiple colours. Fluorescent imaging in small animals is extensively used in cancer research and in investigating protein/protein interaction, and it is found application in BTE. For instance, transgenic mice harbouring GFP under the control of the rat collagen $1 \alpha 12.3 \mathrm{~kb}$ 
Table 2. Imaging modalities for small animals (modified from ref. [71])

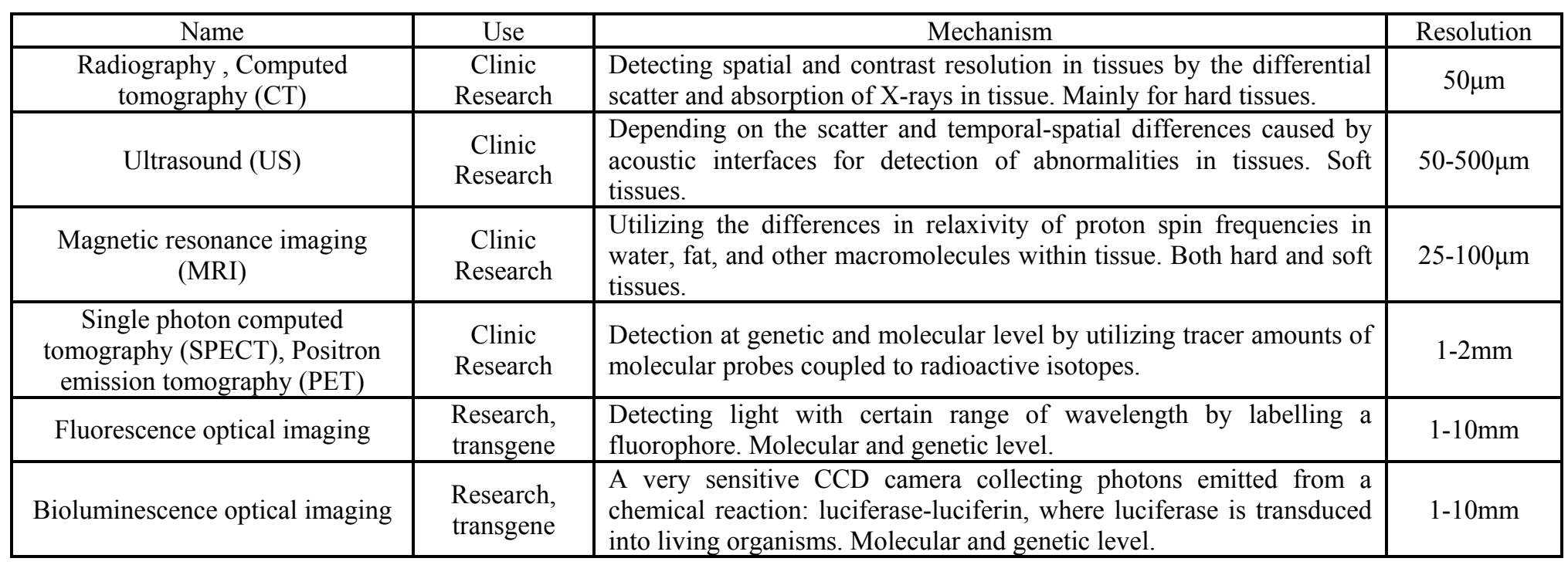


and 3.6kb promoter segments were created [72] and GFP was used to identify the promoter segment from the collagen 1a1 promoter responsible for expression in osteoblasts [73-75].

Similar to fluorescent imaging, bioluminescent imaging (BLI) with luciferase is widely applied to monitor gene expression and cellular activities both in vitro and in vivo, due to the fact that the promoter can be tailored for a gene of interest [76-78]. BLI is based on the luciferase-luciferin reaction, which produces photons which can be captured by very sensitive cooled chargecoupled device (CCCD) camera. Fig. 6 showed the reaction and the facility for BLI. In this reaction, luciferase together with cofactors $\mathrm{Mg}$ and $\mathrm{O}_{2}$ oxidizes luciferin into oxyluciferin and light. When luciferase expression is under the control of the regulatory sequences of a certain interesting gene in cells or animals (most frequently used are rat and mouse), administration of luciferin would result in light emission during the biological process or tissue of interest. In order to capture the light, a black chamber is designed to hold cells or animals, while animals undergo anaesthesia in order to locate the light source. Then a CCCD camera collects the photons and computer software converts the signal into images.

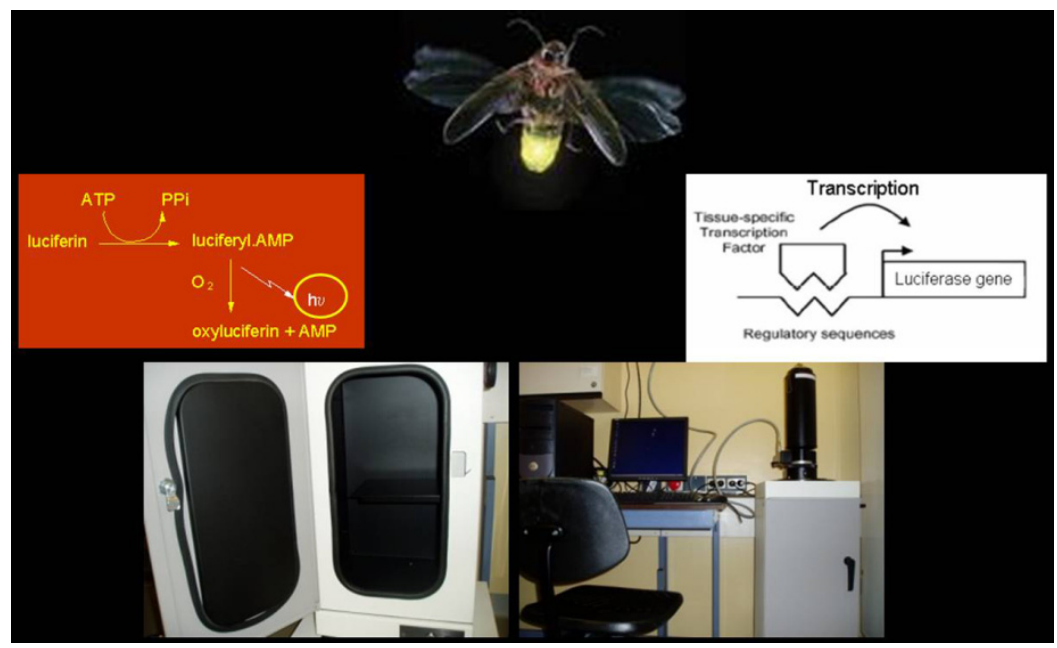

Figure. 6 Firefly luciferase and bioluminescent imaging. Luciferase is an enzyme which facilitates luciferin oxidization in the presence of $\mathrm{Mg}, \mathrm{O}_{2}$ and ATP, thus emitting light which can be captured by a CCCD camera.

Firefly luciferase is the most used due to several advantages. First, its substrate D-luciferin is non-toxic and diffuses within minutes throughout the entire body by intraperitoneal injection in mice. Second, the light intensity is 
high and stable for a long time. The emitted light has a broad spectrum from 500 to $620 \mathrm{~nm}$ with a peak at $\approx 560 \mathrm{~nm}$ which is favourable for penetration of tissues. Without internal background noise (cells do not produce photons), BLI is highly sensitive. Next, because the substrate luciferin is non-toxic, this technique enables repeated imaging of a single organism for a long period of time, thus reducing the sample number and diminishing the internal variation. As such, it serves as a non-invasive, quantitative and repetitive imaging tool on transgenic expression in an individual organism. Except the firefly luciferase, other luciferase sources including Renilla (sea pansy), Gaussia (marine copepod) and bacterial luciferase are also applied in biomedical research [79]. Renilla and Gaussia are ATP-independent and have smaller luciferase, which make them a good candidate. But the two, with coelenterazine as the substrate, produce blue light with an emission peak at around $480 \mathrm{~nm}$, and produce higher background signal caused by auto-oxidation of coelenterazine, thus limiting their application in in vivo detection.

\section{Luciferase transgenic animals and cells}

BLI of luciferase labelled cells and transgenic animals is extensively exploited in multiple fields of biomedical research. Fig. 7 shows an example of the use of BLI in monitoring tumor growth [1]. This mouse received $1 \times 10^{6}$ K1735Br2lucA3 melanoma cells expressing luciferase at day 0 by intravenous injection. Images at day 1, 7, 15 and 19 reveal an increasing light intensity of tumor cells in the lungs. The mouse also exhibited bioluminescence in the tail and the abdomen, indicating the presence of cells at the site where cancer cells spread into the abdominal region. Hypoxia responsive element (HRE)-luciferase cells were reported to detect hypoxia in developing tumors [80]. To summarize, BLI is currently used to investigate cell number, inflammation, apoptosis, vasculogenesis and signal transduction (for a review, see ref. [77]). In BTE, cell proliferation and differentiation can be monitored depending on the promoter driving luciferase. Using the constitutively active cytomegalovirus (CMV) promoter, cell survival and proliferation were examined both in vitro and in vivo $[81,82]$. The activity of several osteoprogenitor-specific promoters was assessed to monitor osteogenic differentiation of stem cells in vitro including the promoters of Runx2/Cbfa-1, osteopontin (OPN), osteocalcin (OCN), collagen type 1a (COL), alkaline phosphatise (ALP) and the bone morphogenetic protein responsive element (BRE) [83, 84]. Vascularisation was evaluated by vascular endothelial growth factor receptor 2 (VEGFR2)-luciferase 
cells and mice $[55,85]$.
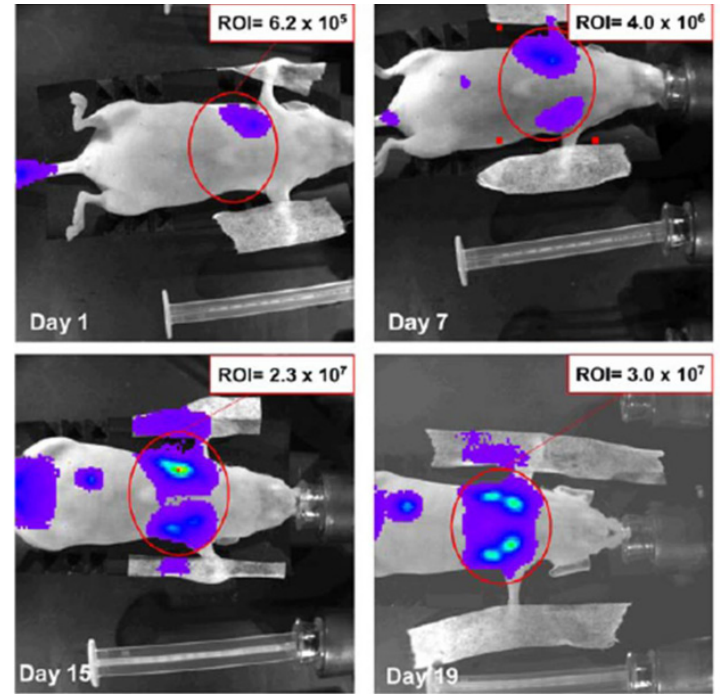

Figure. 7 Non-invasive BLI of an individual mouse in time after intravenous administration of luciferase expressing melanoma cells. This mouse received $1 \times 10^{6}$ K1735Br2lucA3 melanoma cells expressing luciferase at day 0 . Images were taken at days $1,7,15$, and 19 after inoculation of the melanoma cells. The drawn ovals demarcate the region of interest (ROI) that was selected for the quantification of the bioluminescence signal (in photons/s) in the lungs. (Adapted from ref. [1].)

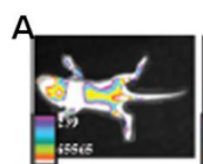

1 week

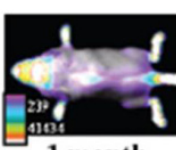

1 month

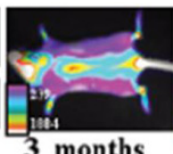

3 months

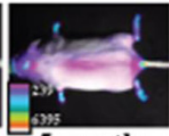

5 months

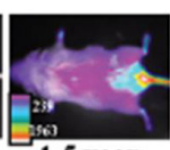

1.5 year
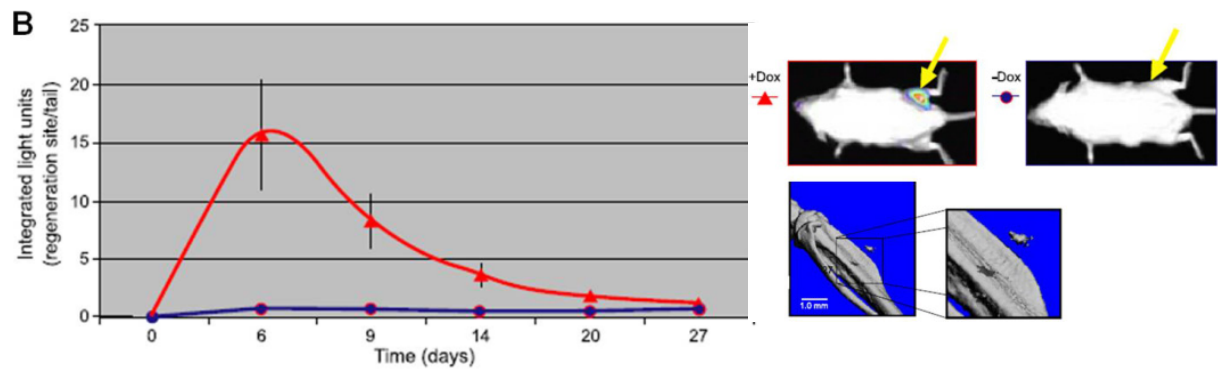

Figure. 8 Bioluminescent imaging of bone development and remodeling in human osteocalcin-luciferase transgenic mice. (A) Luciferase activity dropped during postnatal bone formation. (B) $\mu \mathrm{CT}$ and BLI data on investigating bone formation by hMSCs transduced with rhBMP2 gene. (Adapted from ref $[86,87]$.)

Luciferase transgenic mice are a very attractive tool for understanding gene function in vivo. This transgenic mouse model is applied to investigate bone diseases like arthritis [88-90] and cancer-induced bone metastasis [91-93]. Several transgenic mouse models related to BTE were reported including BMP 4-luciferase mice [94], bone sialoprotein (BSP)-luciferase mice [95], bone specific enhancer of the mouse collagen $\alpha(1)$ I-luciferase mice [96], human 
osteocalcin (hOC)-luciferase mice [97] etc. Osteocalcin, a bone specific gene, is expressed by osteoblasts and hypertropic chondrocytes. hOC-Luc mice expressed strong luciferase activity from skeletal tissues during skeletal development and repair (Fig. 8) [86]. Furthermore, the mice expressed luciferase when ectopic bone formed from hMSCs transduced with rhBMP2 gene in the hind limb muscle [87].

\section{Aims and outlines of this thesis}

The successful application of cell-based bone tissue engineering requires the thorough understanding of every aspect affecting cells growth and differentiation in a direct or indirect way. This thesis includes three parts. The first sub-goal is to improve hMSCs osteogenic commitment in vitro by impinging on signalling pathways using a pharmaceutical approach. The second sub-goal is to use molecular imaging to monitor cell survival and nutrient/oxygen availability in relation to diffusion properties of tissue engineered constructs. The third sub-goal is to evaluate two transgenic mouse models for detecting ectopic bone formation using bioluminescent imaging.

To improve hMSCs proliferation or osteogenic differentiation, we are interested in optimizing the culture protocol by screening compounds impinging on cell signalling pathways for this purpose. It has been reported that the protein kinase C (PKC) signalling pathway is involved in osteoblast proliferation and differentiation [98-100]. Thus, in chapter 2, we have investigated the role of the PKC family in the context of hMSCs osteogenic differentiation in vitro.

When cell culture is transferred from a $2 \mathrm{D}$ tissue culture flask to $3 \mathrm{D}$ tissue engineering (TE) composites, one of the major issues in BTE is to provide sufficient nutrient to cells, because cellular oxygen and nutrient availability is essential for cell survival, growth and even differentiation. Nowadays, a lot of effort is devoted to engineer a vasculature network in tissue engineered grafts to support better cell survival. It is crucial to establish an efficient approach to evaluate cellular oxygen/nutrient availability noninvasively and in time. Chapter 3 demonstrates the possibility to monitor the cellular oxygen level both in vitro and in vivo by introducing a luciferase gene under the control of the hypoxia responsive element.

Furthermore, to get better nutrient provision for cells, understanding the basis of nutrient diffusion within biomaterials is necessary, because this will 
bring very precious knowledge to biomaterials manufacturing and structure design. In Chapter 4, we validated the feasibility to monitor molecular distribution and diffusion in a 3D gel using optical imaging technology.

When hMSCs are implanted in vivo, we expect that hMSCs do not merely deposit new bone in the grafts, but they may also trigger the induction of bone formation of host stem cells. It has for instance been reported that during osteogenic differentiation, hMSCs secrete growth factors and cytokines which stimulate osteogenic differentiation of host progenitor cells. It was demonstrated that approximately $30 \%$ of newly formed bone was from mouse cells when 1 million hMSCs were implanted [101]. As an alternative to the traditional tedious bone evaluation methods, we have assessed the feasibility to use luciferase transgenic mice for this purpose. BLI of luciferase expression is widely applied in many kinds of cellular activity research. Its non-invasive and quantitative properties enable it to be a potential tool for investigating transgenic small animals or cells. In Chapter 5 we evaluated two transgenic mouse lines harbouring luciferase gene under the control of specific osteogenic promoters during bone remodelling and ectopic bone formation.

In Chapter 6, I have discussed the results obtained in the thesis and future perspectives are discussed. 


\section{Chapter 2}

\section{The effect of PKC activation and inhibition on osteogenic differentiation of hMSCs}

Jun Liu ${ }^{1}$, Eugene Someren ${ }^{2,3}$, Anouk Mentink ${ }^{1}$, Ruud Licht ${ }^{1}$, Koen Dechering ${ }^{4}$, Clemens van Blitterswijk ${ }^{1}$, Jan de Boer ${ }^{1}$,

1. MIRA Research Institute, Department of Tissue Regeneration, University of Twente, Enschede, The Netherlands

2. Department of Applied Biology, Radboud University Nijmegen, Nijmegen, The Netherlands

3. Centre for Molecular and Biomolecular Informatics, Radboud University Nijmegen, Nijmegen, The Netherlands

4. Department of Target Discovery, Schering-Plough Research Institute, Oss, The Netherlands 


\section{ABSTRACT}

Human mesenchymal stem cells (hMSCs) are considered for several areas of clinical therapy due to their multipotent nature. For instance, osteogenic hMSCs are applied in bone tissue engineering but current differentiation protocols need further optimization before they can be clinically applied. Protein kinase $\mathrm{C}$ (PKC) family members have been implicated in bone metabolism, which prompted us to use a pharmaceutical approach to manipulate PKC signaling in hMSCs. Inhibition of PKC resulted in a dose-dependent inhibition of dexamethasone-induced osteogenic differentiation. Surprisingly, PKC activation using phorbol 12-myristate 13-acetate (PMA) also resulted in inhibition of osteogenesis, although we observed that inhibition was more pronounced at low than at high concentrations of PMA. Furthermore, we observed that inhibition of PKC $\delta$ blocked alkaline phosphatase (ALP, an early marker of osteogenic differentiation) expression, whereas the inhibition of the conventional PKC subfamily and $\mathrm{PKC} \mu$ using Gö6976 resulted in an induction of ALP activity, collagen (I) expression and mineralization. In conclusion, inhibition of the conventional $\mathrm{PKCs} / \mathrm{PKC} \mu$ and activation of $\mathrm{PKC} \delta$ could further benefit osteogenic differentiation of hMSCs in vitro and in vivo, which is currently under investigation.

\section{INTRODUCTION}

Human mesenchymal stem cells (hMSCs) resident in bone marrow are a type of multipotent cells which can differentiate into adipocytes, osteoblasts, chondrocytes [16], myoblasts [102], and other cell types. In addition, they can be easily isolated and expanded in vitro. Based on these advantages, hMSCs are widely employed in tissue engineering and regenerative medicine. For bone tissue engineering, hMSCs can differentiate into osteoblasts under proper conditions, evidenced by elevated osteogenic markers such as alkaline phosphatase (ALP) and deposition of a mineralized extracellular matrix in vitro. Furthermore, hMSCs can form ectopic bone tissue in vivo [103, 104]. Thus, hMSCs may serve as a cell source for bone regeneration. However, the clinical bone defect can be as large as centimeters, which demands a large number of 
hMSCs. We previously reported that hMSC expansion in vitro results in loss of multipotency [23] and the in vivo bone forming capacity is not sufficient to bridge large bone defects [11]. Therefore, we are looking for possibilities to stimulate hMSC proliferation and osteogenic differentiation by interfering with signal transduction pathways $[27,105,106]$. For instance, we recently reported that activation of the protein kinase A signal transduction pathway results in enhanced osteogenic differentiation in vitro and bone formation in vivo [28]. In this report, we focused on the potential to improve the performance of hMSCs by interfering with protein kinase $\mathrm{C}$ (PKC) signaling.

PKC is a family of serine/threonine protein kinases, which is involved in different cellular functions through different downstream signaling cascades [107]. Typically, PKC signaling is activated by binding of extracellular ligands to so-called G-protein coupled receptors (GPCR, see Fig. 1 for an overview) and subsequent activation of Gq-proteins. This results in the activation of phospholipase C (PLC) and hydrolysis of phosphatidylinositol bisphosphate (PIP2) to inositol trisphosphate (IP3) and diacylglycerol (DAG). IP3 releases calcium ions from the endoplasmic reticulum (ER) into the cytoplasm. $\mathrm{Ca}^{2+}$ and DAG are co-factors which activate PKC isozymes by inducing their translocation to the membrane [108]. The PKC family has 11 isoforms in 3 classes: the conventional calcium- and DAG-dependent class $(\alpha, \beta I$, $\beta I I$, and $\gamma$ isoforms), the novel calcium-independent and DAG-dependent class $(\theta, \eta, \delta$, $\varepsilon$ and $\mu$ ) and the atypical calcium- and DAG-independent class $(\zeta$ and $\lambda / \mathrm{t}$ isoforms) $[109,110]$. In addition, $\mathrm{PKC} \mu$ contains a cysteine-rich motif longer than that of other PKCs, and is referred to as PKD. It is ubiquitously expressed and involved in diverse cellular activities [111]. Some PKC isoforms $(\alpha, \beta I$, $\varepsilon, \zeta, \lambda)$ have been identified in osteoblasts [112], and PKC has been reported to be involved in bone remodeling by affecting both osteoblasts and osteoclasts [113-116]. In addition, PKC plays a critical role in the modulation of osteoprotegerin (OPG) and vascular endothelial growth factor (VEGF) expression and activity in human osteoblasts and endothelial cells $[115,117$, $118]$. 


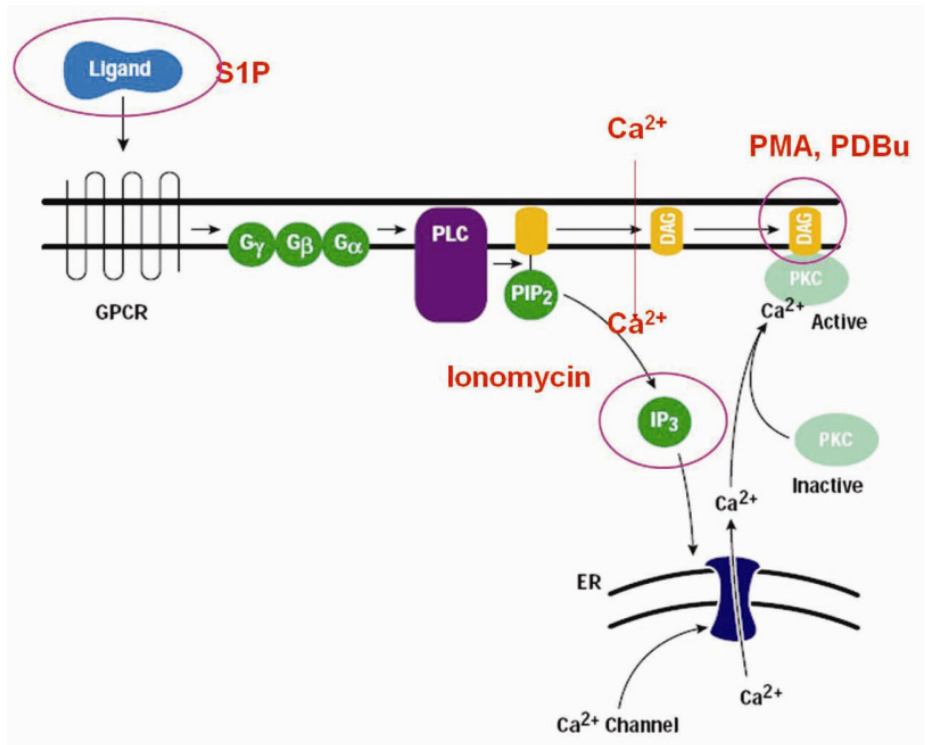

Figure 1. Protein Kinase C signaling pathway. External ligands activate a G-Protein-Coupled Receptor, which activates a G-protein. The G-protein activates phospholipase C (PLC), which cleaves phospho-inositol4,5-bisphosphate $\left(\mathrm{PIP}_{2}\right)$ into 1,2-diacylglycerol (DAG) and inositol-1,4,5-triphosphate $\left(\mathrm{IP}_{3}\right)$. $\mathrm{IP}_{3}$ interacts with a calcium channel in the endoplasmic reticulum (ER), releasing $\mathrm{Ca}^{2+}$ into the cytoplasm. The increase in $\mathrm{Ca}^{2+}$ levels activates PKC, which translocates to the membrane by anchoring to DAG and phosphatidylserine. Sphingosine-1-phosphate (S1P) is the ligand used in this study known to activate PKC. Phorbol 12-myristate 13-acetate (PMA) and phorbol 12,13-dibutyrate (PDBu) are activators of PKC by mimicking DAG. Ionomycin can mimick $\mathrm{IP}_{3}$ by increasing the intracellular $\mathrm{Ca}^{2+}$ level.

Parathyroid hormone (PTH) has an anabolic effect on bone formation by stimulating proliferation and differentiation of osteoblast precursors and promoting the synthetic activities of mature osteoblasts [119]. The positive effect of PTH on differentiation is supposed to be mediated by the PKA signaling pathway while its effect on proliferation is thought to be mediated by PKC $[120,121]$. A role of PKC in cellular proliferation has been delineated in a number of osteogenic cell types. Siddhanti et al. demonstrated that PMA stimulates MC3T3 cell proliferation by PKC activation [122]. In addition, the transforming growth factor- $\beta$ (TGF- $\beta$ ) stimulates MC3T3 proliferation by local release of the GPCR ligand prostaglandin E2 (PGE2) through a PKC/ERK signaling cascade [123]. PMA is known to stimulate cell proliferation in cultured mouse calvaria and fetal rat osteoblasts [124]. Both PTH and parathyroid hormone-related peptide (PTHrP) stimulate rat primary osteoblast proliferation via the PKC/ERK pathway [125], and PKC-dependent activation of the Ras and MAPK signaling pathways [126] respectively. PGE2 stimulates human bone cells by inducing PLC $(\mathrm{Ca} / \mathrm{PKC})$ [127]. Furthermore it has been 
demonstrated that the PKC $\alpha$-isoform is involved in proliferation of primary human osteoblasts [98].

PKC also plays a role in osteogenic differentiation in a number of different cell types. Reported activities include regulation of Runx2 expression and activity [99], increasing the expression of BMP receptor IA [128], stimulating fibronectin expression $[129,130]$ enhancing N-Cadherin expression [131], and increasing indexes of the osteoblast phenotype in MC3T3, C2C12, C3H10T1/2, M2-10B4 mouse bone marrow stromal cells [132], primary cultured rat osteoblasts and human calvaria osteoblasts.

In conclusion, PKC seems to play a role in both proliferation and differentiation of osteoblasts and murine mesenchymal stem cells. In this article, we used a pharmacological approach to investigate whether PKC activation and inhibition could be used to enhance proliferation and differentiation of hMSCs.

\section{MATERIALS AND METHODS}

\section{Chemicals}

Phorbol 12-myristate 13-acetate (PMA), phorbol 12,13-dibutyrate $(\mathrm{PDBu})$, ionomycin, sphingosine-1-phosphate (S1P), calphostin $\mathrm{C}$ and staurosporine were purchased from Sigma-Aldrich. Gö6976, rottlerin and pseudo-substrate inhibitor of PKC $\theta$ were purchased from Calbiochem. Antibodies for specific PKC isozymes were purchased from Cell Signaling.

\section{Isolation and culture of hMSCs}

Bone marrow aspirates $(5-20 \mathrm{ml})$ were obtained from donors with written informed consent. hMSCs were isolated and proliferated as described previously [133]. Briefly, aspirates were resuspended using 20G needles, plated at a density of $5 \times 10^{5}$ cells $/ \mathrm{cm}^{2}$ and cultured in hMSC basic medium containing $\alpha$-minimal essential medium ( $\alpha$-MEM, Gibco), $10 \%$ fetal bovine serum (FBS, Cambrex), 0.2mM ascorbic acid (Asap, Gibco), 2mM L-glutamine (Gibco), $100 \mathrm{U} / \mathrm{ml}$ penicillin (Gibco) and $10 \mu \mathrm{g} / \mathrm{ml}$ streptomycin (Gibco). Cells were grown at $37^{\circ} \mathrm{C}$ in a humid atmosphere with $5 \% \mathrm{CO}_{2}$. Medium was refreshed twice a week and cells were used for further subculturing or cryopreservation upon reaching near confluence. HMSC osteogenic medium was composed of hMSC basic medium supplemented with $10^{-8} \mathrm{M}$ dexamethasone (dex, Sigma) and hMSC mineralization medium was composed of basic medium 
supplemented with $10^{-8} \mathrm{M}$ dex and $0.01 \mathrm{M} \beta$-glycerophosphate (Sigma). The hMSCs used in the study were from donors ranging between 25 and 80 years of age. All experiments in the manuscript have been repeated with cells from at least 3 donors.

\section{Microarray analysis}

To study the expression of PKC isozymes at the transcriptional level, hMSCs at passage 2 from 72 donors were grown in basic medium. RNA was isolated using an RNeasy midi kit (Qiagen) and $8 \mu \mathrm{g}$ of total RNA was used for probe labeling according to the manufacturer's protocol (Affymetrix). The probe quality was verified using lab-on-chip technology (Agilent Technologies) and samples were hybridized to Human Genome Focus arrays according to the manufacturer's protocol (Affymetrix). Data were quantified using GCOS 1.2 software (Affymetrix). Normalization and statistical analysis of the data was performed using the error model developed for Affymetrix GeneChips [134], carried out using Rosetta Resolver Version 4.0. Besides normalized expression values, this error model returns for each probe-set on each array, a p-value that reflects the probability that the expression value originates from background noise rather than mRNA abundance.

\section{Proliferation assay}

hMSCs were seeded at 1000 cells $/ \mathrm{cm}^{2}$ and incubated with different reagents for the denoted time periods. Each experiment was performed in triplicate. Cell proliferation was determined using Alamar blue assay kit (Bioscience). The Alamar blue solution was diluted 1:10 in culture medium, and cells were cultured for 4 hours. $200 \mu$ l of Alamar blue solution from each well was transferred into 96-well plates, and fluorescence was measured using a Perkin Elmer Luminescence Spectrometer LS50B. Results were recorded by FL winlab software and the data were subtracted with background value from a well without cells.

\section{ALP analysis by flow cytometry (FACS)}

hMSCs were seeded at 1000 cells $/ \mathrm{cm}^{2}$ and incubated with different reagents for the denoted time periods. Each experiment was performed in triplicate with a negative control (cells grown in basic medium), a positive control (cells grown in osteogenic medium) and experimental conditions. At the end of the culture period, the cells were trypsinized and incubated for 30 minutes in block buffer (PBS with 5\% bovine serum albumin (BSA) [Sigma] 26 
and $0.05 \% \mathrm{NaN}_{2}$ ), then incubated with primary antibody (anti-ALP, B4-78 [Developmental Studies Hybridoma Bank, University of Iowa, USA]) diluted in wash buffer (PBS with $1 \%$ BSA and $0.05 \% \mathrm{NaN}_{2}$ ) for 30 minutes or with isotype control antibodies. Cells were then washed three times with wash buffer and incubated with secondary antibody (goat anti mouse IgG PE, DAKO) for 30 minutes. Cells were washed three times and suspended in $50 \mu \mathrm{l}$ wash buffer with $10 \mu 1$ Viaprobe (Pharmingen) for live/dead cell staining and only living cells were used for further analysis. ALP expression levels were analyzed on a FACS Calibur (Becton Dickinson Immuno cytometry systems).

\section{Biochemical ALP assay}

hMSCs were seeded at 1000 cells/well in 96-well plates and incubated with different reagents for the denoted time periods. Each experiment was performed in triplicate with a negative control (cells grown in basic medium), a positive control (cells grown in osteogenic medium) and experimental conditions. Samples were lysed with $0.2 \%$ Triton 100/PBS and alkaline phosphatase activity was determined in $100 \mu \mathrm{l}$ aliquots of clarified cell lysate with $100 \mu$ of $20 \mathrm{mM}$ pNPP ( $p$-Nitrophenyl phosphate, Sigma-Aldrich) as a substrate. The resulting absorbance at $450 \mathrm{~nm}$ was recorded after $30 \mathrm{~min}$ incubation at $37{ }^{\circ} \mathrm{C}$, corrected for background noise and normalized to the Alamar blue values.

\section{Mineralization, Von Kossa staining and calcium deposition quantification}

For mineralization, hMSCs were seeded in triplicate at $5000 \mathrm{cells} / \mathrm{cm}^{2}$ in T25 culture flasks and incubated in basic and mineralization media for 28 days treated with $10 \mathrm{nM}$ and 1000nM PMA for 4 days or 28 days, and with 100nM Gö6976 for 20 days. Mineralization medium was used as a positive control and basic medium as a negative control. The total calcium deposition was assayed using a calcium assay kit (Sigma diagnostics; 587A) according to the manufacturer's protocol. Briefly, the culture medium was aspirated, washed twice with calcium- and magnesium-free PBS (Gibco) and incubated overnight with $0.5 \mathrm{~N} \mathrm{HCl}$ on an orbital shaker at room temperature. The supernatant was collected for direct measurement or stored at $-20^{\circ} \mathrm{C}$. The calcium content was measured at $575 \mathrm{~nm}$ (Perkin Elmer, Lamda 40) and expressed as mg calcium/flask. For von Kossa staining, cells were washed with PBS (Gibco), fixed with $4 \%$ para-formaldehyde (Sigma) and stained with $5 \% \mathrm{AgNO}_{3}$ (Sigma) in dematerialized water under mild UV exposure. 


\section{RNA isolation and quantitative PCR}

The effect of Gö6976 and PMA on expression of osteogenic marker genes was analyzed by seeding hMSCs at 5000 cells $/ \mathrm{cm}^{2}$ in $\mathrm{T} 75$ flasks supplemented with various medium compositions for 5-6 days. Total RNA was isolated using an Rneasy mini kit (Qiagen) and on-column DNase treated with 10U RNase free DNase I (Invitrogen) at room temperature for 30 minutes. DNAse was inactivated at $72^{\circ} \mathrm{C}$ for 15 minutes. The quality and quantity of RNA were analyzed by gel electrophoresis and spectrophotometry (ND-1000 spectrophotometer). $1 \mu \mathrm{g}$ of RNA was used for first strand cDNA synthesis using Superscript II (Invitrogen) according to the manufacturer's protocol. One $\mu 1$ of $100 \mathrm{x}$ diluted cDNA was used for 18s rRNA amplification and $1 \mu 1$ of undiluted cDNA was used for the other genes. PCR was performed on a Light Cycler real time PCR machine (Roche) using SYBR green I master mix (Invitrogen). Data was analyzed using Light Cycler software version 3.5.3, using the fit point method by setting the noise band to the exponential phase of the reaction to exclude background fluorescence. Expression of osteogenic marker genes are calculated relative to $18 \mathrm{~s}$ rRNA levels by the comparative $\Delta \mathrm{CT}$ method [135]. The primers used in the study are listed in Table 1.

Table 1. The primers used for Q-PCR.

\begin{tabular}{|c|c|}
\hline $\begin{array}{c}C O L 1 A 1 \\
\text { (Collagen type I) }\end{array}$ & $\begin{array}{l}\text { F 5', agggccaagacgaagacatc 3, } \\
\text { R 5, agatcacgtcatcgcacaaca 3, }\end{array}$ \\
\hline $18 s$ rRNA & $\begin{array}{l}\text { F 5' cggctaccacatccaaggaa } 3^{\prime} \\
\text { R 5, gctggaattaccgeggct } 3{ }^{\prime}\end{array}$ \\
\hline $\begin{array}{c}B G L A P \\
\text { (Osteocalcin) } \\
\end{array}$ & $\begin{array}{l}\text { F 5', ggcagcgaggtagtgaagag 3, } \\
\text { R 5, gatgtggtcagccaactcgt 3, }\end{array}$ \\
\hline $\begin{array}{c}S P P 1 \\
\text { (Osteopointin) } \\
\end{array}$ & $\begin{array}{l}\text { F 5', ccaagtaagtccaacgaaag } 3^{\prime} \\
\text { R 5, ggtgatgtcctcgtctgta 3, }\end{array}$ \\
\hline $\begin{array}{c}\text { Alp } \\
\text { (Alkaline phosphotase) } \\
\end{array}$ & $\begin{array}{l}\text { F 5', cggctaccacatccaaggaa } 3^{\prime} \\
\text { R 5, gctggaattaccgcgget 3, }\end{array}$ \\
\hline
\end{tabular}

\section{Western blotting}

hMSCs were cultured in 10nM and 1000nM PMA for 4 hours, and total protein was isolated and quantified using the BCA protein assay kit (Pierce). The cell lysates were separated using sodium dodecyl sulfate polyacrylamide gel electrophoresis (SDS-PAGE) and transferred to Immobilon-P membranes. The membranes with transferred proteins were blocked with TBS (Trisbuffered saline) with 5\% milk for 1 hour at room temperature (RT), and probed with specific primary antibodies diluted to the recommended concentrations for 4 hours at RT. Next, the membranes were incubated with horseradish 28 
peroxidase (HRP) conjugate goat anti-rabbit IgG (DAKO) as the secondary antibody for 1 hour at RT. Protein detection was performed by luminescence using a Kodak image station 4000MM after incubating the membranes with Supersignal chemiluminescent detection (Pierce) for 5 minutes.

\section{Statistical analysis}

Statistical analyses were performed using one-way ANOVA. ${ }^{*} p<0.05$, $* * p<0.01, * * * p<0.001$.

\section{RESULTS}

\section{Gene expression of PKC isozymes in human mesenchymal stem cells}

The PKC family comprises 11 isozymes in 3 classes which display differential expression [136] but their expression in hMSCs has not been described so far. We performed genome-wide gene expression analysis on hMSCs from 72 donors (manuscript in preparation) and focused on PKC isozyme expression. To assess whether a gene was significantly expressed above noise, we employed a threshold of $5 \%$ on the p-values obtained from the error model from Rosetta Resolver (Fig. 2A). All 72 analyzed donors significantly expressed PRKD1 (PKC $\mu$ ) and PRKD3 (PKCv), whereas no expression above noise could be detected for PRKCG (PKC $\gamma)$. PRKCA, PRKCD, $P R K C H, P R K C I$ and PRKCE (PKC $\alpha, \delta, \eta, \lambda / \imath$ and $\varepsilon$ ) expression could be detected in most but not all donors. In addition, the normalized gene expression for the various isoforms varied between individual donors determined by fluorescent signal in Fig. 2B, supporting our previous data about inter-donor variation [23].

\section{PKC activation has no positive effects on proliferation and osteogenic differentiation of hMSCs}

We assessed whether any of the reagents known to activate the PKC pathway could be used to stimulate hMSC proliferation and osteogenic differentiation (Fig.1). S1P is a GPCR ligand known to activate PKC, PMA and $\mathrm{PDBu}$ are broad activators of PKC by mimicking DAG, and ionomycin can mimic $\mathrm{IP}_{3}$ by increasing the intracellular $\mathrm{Ca}^{2+}$ level. After 6 days of incubation in both basic and osteogenic media, S1P had no significant effect on either proliferation or ALP expression of hMSCs (Table 2). 

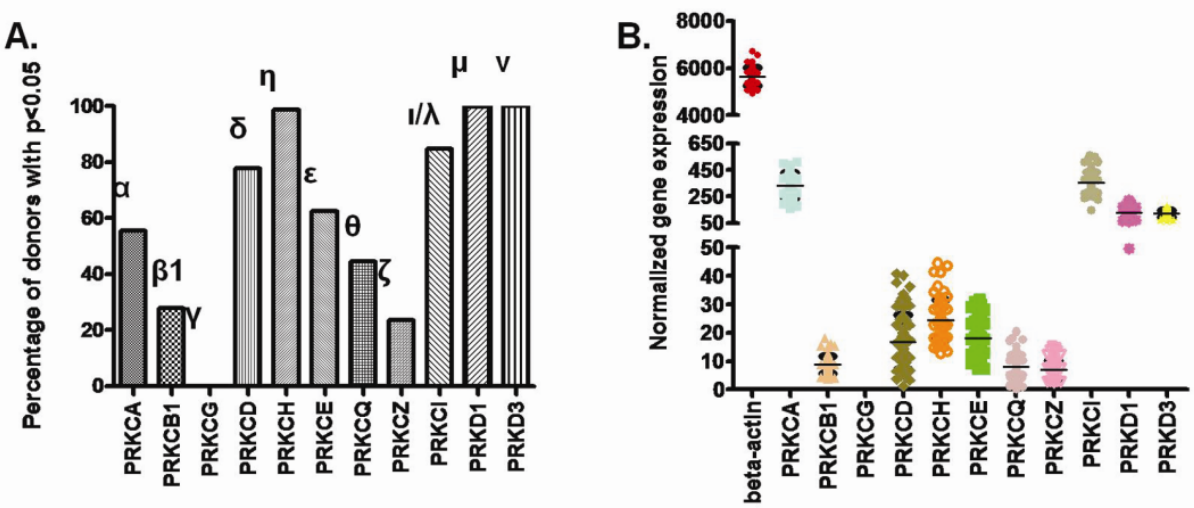

Figure 2. Expression profile of PKC isozymes in hMSCs from 72 donors. (A) The percentage of donors with a p-value $<0.05$ from each isozyme out of 72 donors. The p-values of PKC genes were analyzed by comparing with background noise. $\beta$-actin was taken as the reference gene. (B) The absolute fluorescence intensity distribution of PKC isozymes in hMSCs from 72 donors.

PMA stimulates both the conventional and novel families of PKC, evidenced by increased phosphorylation of PKC isozymes upon exposure of hMSCs to the compound (Fig. 3A), which resulted in an inhibition of hMSC proliferation and osteogenic differentiaton. Interestingly, the inhibition was most profound at low concentrations of PMA but became milder at higher concentrations (Fig. 3B, C and D). We also tested an even higher concentration of PMA $(10 \mu \mathrm{M})$, but it still had the inhibitory effect (data not shown). To further demonstrate that PKC activation inhibits osteogenic differentiation, we analyzed the effect of PMA on $A L P$ and COL1A1 mRNA expression (Fig. 3E), and on hMSC mineralization (Fig. 3F). Both ALP expression and COL1A1 expression were inhibited by PMA, and the effect was more pronounced at 10 nM PMA than at $1000 \mathrm{nM}$. In addition, both 4 and 28 days exposure of hMSCs to both concentrations of PMA inhibited dexamethasone-induced calcium deposition. To confirm that DAG-mediated signaling has a concentrationdependent effect on hMSC proliferation and osteogenesis, we also tested the effect of PDBu. Similar to PMA, we observed a bi-phasic response with strong inhibition of proliferation and ALP induction at low concentrations and mild inhibition at high concentrations (Table 2).

The conventional PKC family signals both through DAG and $\mathrm{Ca}^{2+}$, in contrast to the novel family, which signals only through DAG. Therefore, we exposed hMSCs to the calcium-mimetic ionomycin, which resulted in a dosedependent decrease in cell proliferation and ALP expression (Table 2). In 
conclusion, none of the tested PKC activators could be used to augment proliferation or differentiation of hMSCs.

Table 2. The compounds used and their effects on hMSCs proliferation and osteogenic differentiation*.

\begin{tabular}{|c|c|c|c|c|}
\hline & $\begin{array}{l}\text { Compound } \\
\text { name }\end{array}$ & $\begin{array}{l}\text { Concen- } \\
\text { tration }\end{array}$ & Proliferation ${ }^{1}$ & ALP activity \\
\hline Ligand & $\begin{array}{c}\text { Sphingosine- } \\
\text { 1-phosphate } \\
\text { (S1P) }\end{array}$ & $1-1000 \mathrm{nM}$ & Not influenced & Not influenced ${ }^{2}$ \\
\hline \multirow{3}{*}{$\begin{array}{l}\text { Activator } \\
\text { (Second } \\
\text { messenger } \\
\text { mimic) }\end{array}$} & $\begin{array}{l}\text { phorbol 12- } \\
\text { myristate 13- } \\
\text { acetate } \\
\text { (PMA) }\end{array}$ & $1-1000 \mathrm{nM}$ & $\begin{array}{l}\text { Inhibition at low } \\
\text { concentrations (10 } \\
\mathrm{nM}) \text { while mild } \\
\text { inhibition at high } \\
\text { concentrations }\end{array}$ & $\begin{array}{l}\text { Inhibition at low } \\
\text { concentrations (10 } \\
\mathrm{nM}) \text { while mild } \\
\text { inhibition at high } \\
\text { concentrations }^{2}\end{array}$ \\
\hline & $\begin{array}{l}\text { phorbol } \\
12,13- \\
\text { dibutyrate } \\
(\mathrm{PDBu})\end{array}$ & $1-1000 \mathrm{nM}$ & $\begin{array}{l}\text { Inhibition at low } \\
\text { concentrations } \\
(100 \mathrm{nM}) \text { while } \\
\text { mild inhibition at } \\
\text { high } \\
\text { concentrations }\end{array}$ & $\begin{array}{l}\text { Inhibition at low } \\
\text { concentrations } \\
(100 \mathrm{nM}) \text { while } \\
\text { mild inhibition at } \\
\text { high } \\
\text { concentrations }^{2}\end{array}$ \\
\hline & Ionomycin & $1-1000 \mathrm{nM}$ & $\begin{array}{c}\text { Dose-dependent } \\
\text { inhibition from } \\
100 \mathrm{nM} \text { on } \\
\end{array}$ & $\begin{array}{c}\text { Dose-dependent } \\
\text { inhibition from } 100 \\
n M \text { on }^{3}\end{array}$ \\
\hline \multirow{2}{*}{$\begin{array}{l}\text { General } \\
\text { inhibitor }\end{array}$} & Staurosporine & $0.1-10 \mathrm{M}$ & $\begin{array}{c}\text { Dose-dependent } \\
\text { inhibition }\end{array}$ & $\begin{array}{c}\text { Dose-dependent } \\
\text { inhibition }^{2}\end{array}$ \\
\hline & Calphostin C & $1-100 \mathrm{nM}$ & $\begin{array}{c}\text { Dose-dependent } \\
\text { inhibition }\end{array}$ & $\begin{array}{l}\text { Dose-dependent } \\
\text { inhibition }^{3}\end{array}$ \\
\hline \multirow{3}{*}{$\begin{array}{l}\text { Specific } \\
\text { inhibitor }\end{array}$} & Gö6976 & $1-1000 \mathrm{nM}$ & $\begin{array}{l}\text { Dose-dependent } \\
\text { inhibition }\end{array}$ & $\begin{array}{c}\text { Stimulated ALP } \\
\text { expression until } \\
100 \mathrm{nM} \text {; inhibited } \\
\text { ALP at } 1000 \mathrm{nM}^{2}\end{array}$ \\
\hline & Rottlerin & $0.5-5 \mu \mathrm{M}$ & $\begin{array}{c}\text { Dose-dependent } \\
\text { inhibition }\end{array}$ & $\begin{array}{c}\text { Dose-dependent } \\
\text { inhibition }^{2}\end{array}$ \\
\hline & $\begin{array}{l}\text { Theta pseudo- } \\
\text { substrate } \\
\text { inhibitor }\end{array}$ & $0.1-10 \mu \mathrm{M}$ & Not influenced & Not influenced $^{3}$ \\
\hline
\end{tabular}

* The experiments were performed in both basic medium with and without dex.

1: Proliferation was assessed by Alamar blue assay; osteogenic differentiation was assessed by ALP activity induction compared to cells grown in medium without treatment.

2: ALP activity was determined by flow cytometry (FACS), expressing the average ALP signal per cell.

3: Relative ALP activity was determined by ALP biochemical assay normalized to cell number and then the ratio was normalized to the basic control. 
A
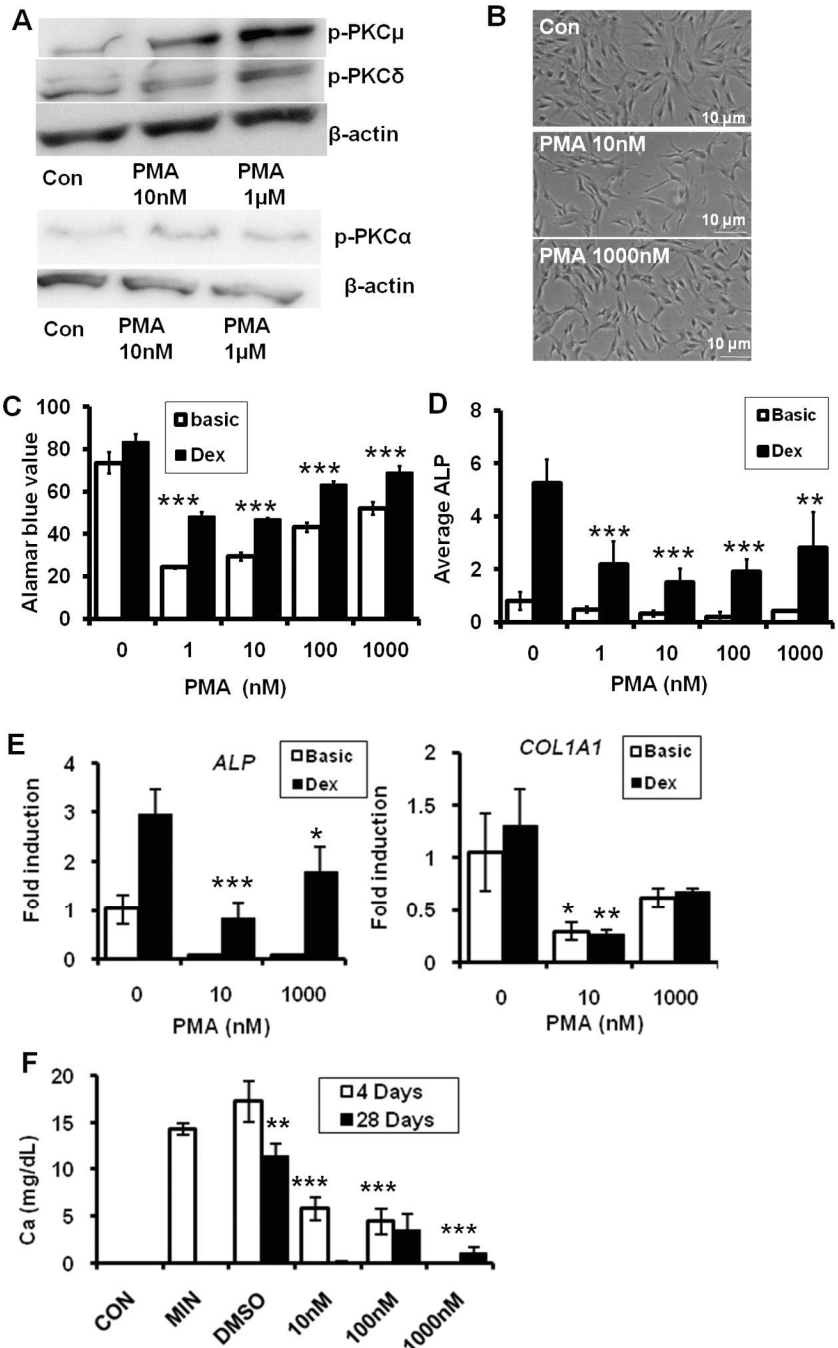

B

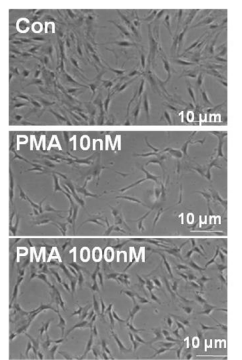

Figure 3. Effect of PKC activation on hMSC proliferation and osteogenic differentiation. (A) Western blot analysis of hMSCs cultured in the presence of 0 , 10 and 1000 nM PMA for 4 hours. The results were confirmed with cells from a second donor. (B) Light microscopy pictures with $100 x$ magnification demonstrated hMSC proliferation in basic medium plus different concentration of PMA after 4 days. (C) The effect of PMA on hMSCs proliferation was expressed as Alamar blue values after 7 days culturing. (D) ALP expression was analyzed using FACS after 7 days culture in the presence of PMA. (E) Gene expression of osteogenic markers COL1A1 and $A L P$ by Q-PCR after 5 days culturing. Expression is indicated as fold-induction compared to cells grown in basic medium and normalized to $18 \mathrm{~s}$ rRNA. (F) hMSCs were cultured with PMA for 4 days (open bar) or 28 days (black bar) in mineralization medium. At the end of the 28-day culture period total calcium deposition

was determined. 1000x diluted DMSO was taken as a solvent control for 1000nM PMA. Error bars indicate standard deviation. Basic, basic hMSC medium; dex; osteogenic medium. Statistical significance is calculated relative to cells grown in media without test compounds.

\section{Divergent effects of different PKC isozyme inhibitor on hMSC differentiation}

The effect of PKC inhibition on hMSCs was analyzed by exposing them to a concentration range of calphostin $\mathrm{C}$ and staurosporin, both general inhibitors of PKC. The two compounds dose-dependently inhibited cell proliferation and ALP expression after 6 days incubation (Table 2). The effect was observed both in basic and osteogenic media.

Next, we tested compounds with a more specific inhibitory profile. Gö6976 is a selective inhibitor of PKCs including PKC $\alpha$ (IC50 = 2.3nM), 
PKC $\beta I(I C 50=6.2 n M)$ and PKC $\mu(\operatorname{IC} 50=20 \mathrm{nM})$ according to the information provided by the manufacturer. We observed no effect on proliferation of hMSCs up to $100 \mathrm{nM}$ of Gö6976 (Fig. 4A). At 1000nM, Gö6976 inhibited proliferation of hMSCs and exerted a profound effect on cell shape (compare cells in Fig. 4C to Fig. 3B). Interestingly, ALP expression was significantly stimulated at $100 \mathrm{nM}$ in both basic and osteogenic media (Fig. 4B). Exposure of hMSCs to $100 \mathrm{nM}$ Gö6976 in osteogenic medium resulted in a 2-fold up-regulation of COL1A1 and ALP expression (Fig. 4D) and it enhanced calcium-deposition in mineralization medium (Fig. 4E). The expressions of other osteogenic marker genes, such as BGLAP and SPP1 were not affected by Gö6976 (data not shown).

To inhibit the novel members of PKC $\delta$ and PKC $\theta$, hMSCs were treated with rottlerin, which have an IC50 of 3-6 $\mu \mathrm{M}$ according to the manufacturer. Rottlerin treatment resulted in a significant reduction of both proliferation and ALP expression (Fig. 5A and B). In addition, we observed that rottlerin treatment changed hMSC morphology from its typical spindle-shaped fibroblastic morphology observed in proliferating hMSCs to a triangular shape (Fig. 5C), which is consistent with reports stating that inhibition of $\mathrm{PKC} \delta$ changes the morphology of hMSCs by altering the cytoskeleton $[137,138]$. To elucidate which isozyme is involved in this effect, we exposed hMSCs to a PKC $\theta$ pseudosubstrate, which inhibits PKC $\theta$ activity but does not affect PKC $\delta$ activity. PKC $\theta$ pseudosubstrate did not exert a significant effect on either osteogenic differentiation, proliferation or cell shape at concentrations ranging from $1 \mathrm{nM}$ to $10 \mu \mathrm{M}$ (Table 2), suggesting that $\mathrm{PKC} \delta$ activity is responsible for maintenance of the osteogenic phenotype. To confirm the latter, we exposed hMSCs to both the PKC $\delta / \theta$ inhibitor rottlerin and the conventional PKC/PKC $\mu$ inhibitor Gö6976. As depicted in Fig. 5D rottlerin inhibited the ALP-inducing effect of Gö6976. Moreover, the presence of rottlerin overruled the recovery of the inhibitive effect of PMA at high concentrations (Fig. 5E). 

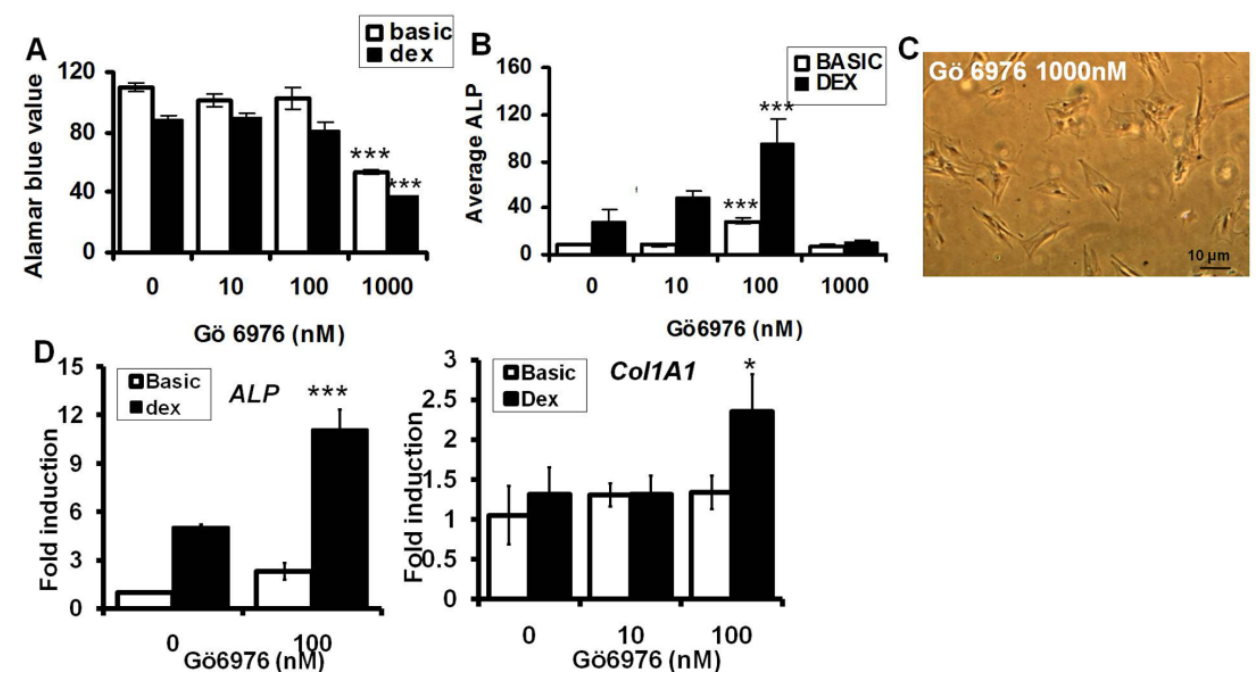

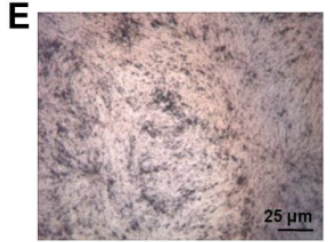

Dex alone

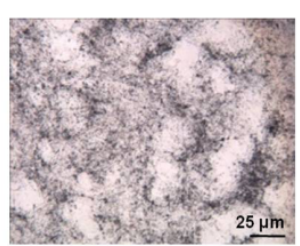

Gö6976 100nM+Dex

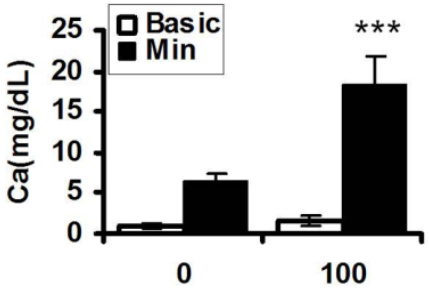

Gö6976 (nM)

Figure 4. Gö6976 stimulates hMSC osteogenic differentiation. (A) hMSC proliferation in the presence of different concentrations of Gö6976 after 5 days. (B) Gö6976 stimulates ALP expression after 5 days. (C) Light microscopy picture with 100x magnification shows the morphology of hMSCs in basic medium with 1000nM Gö6976. (D) CoL1A1 and $A L P$ expression in Gö6976-treated cells expressed as fold-induction compared to untreated control cells. (E) hMSCs were cultured in mineralization medium with 100nM Gö6976 for 20 days. Phase contrast light microscopy pictures with 40x magnification shows enhanced hMSCs mineralization in the presence of Gö6976 by von Kossa staining. Error bars indicate standard deviation. Basic, basic hMSC medium; dex, osteogenic medium. Statistical significance is calculated relative to cells grown in media without test compounds.

\section{DISCUSSION}

In this manuscript we have investigated whether manipulation of PKC activity has an effect on osteogenesis and proliferation of hMSCs. The PKC family has 11 isozymes, which have different tissue-specific expression patterns [136]. In our study, we were unable to detect PRKCG (PKC $\gamma$ ) expression in hMSCs, which is in line with other reports showing that the isoform is mostly 


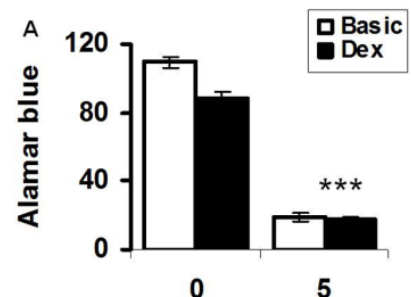

Rottlerin $(\mu \mathrm{M})$

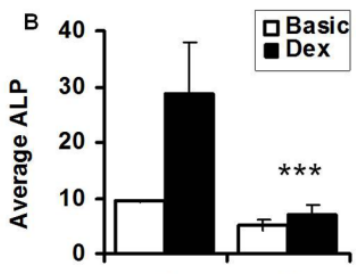

5

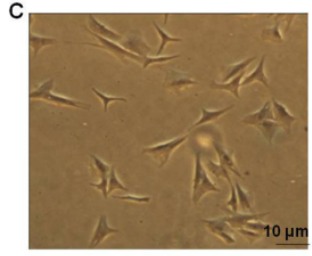

Rottlerin $5 \mu \mathrm{M}$

Rottle rin $(\mu \mathrm{M})$
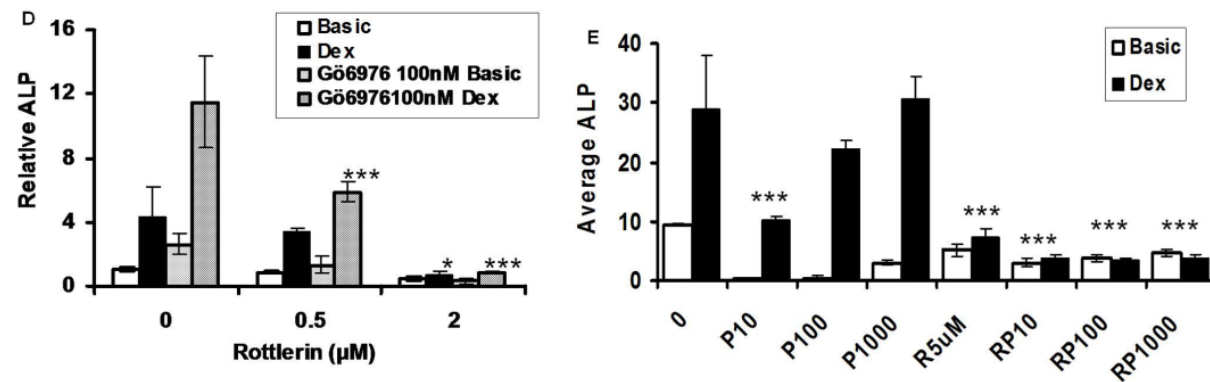

Figure 5. Rottlerin inhibits hMSC proliferation and osteogenic differentiation. (A) Rottlerin inhibited proliferation of hMSCs after 5 days. (B) ALP expression by flow cytometry after 5 days. (C) Light microscopy image at 100x magnification of hMSCs treated with rottlerin after 5 days. (D) Rottlerin inhibited Gö6976 induced ALP determined by the ALP assay after 4-day culturing. (E) ALP expression by hMSC treated with PMA plus or minus $\mu \mathrm{M}$ Rottlerin for 5 days. P10 $=$ PMA $10 \mathrm{nM}$, etc; R $5 \mu \mathrm{M}=$ rottlerin $5 \mu \mathrm{M}$; $\mathrm{RP} 10=$ rottlerin $5 \mu \mathrm{M}+$ PMA $10 \mathrm{nM}$, etc. Error bars indicate standard deviation. Basic means basic hMSC medium; dex means osteogenic medium. Statistical significance is calculated relative to cells grown in media without test compounds.

expressed in brain tissue [139]. Furthermore, PRKD1 and PRKD3 (PKC $v$ and $\mu$ ) were expressed in hMSCs of all 72 donors tested, whereas the expression of the other isoforms were donor dependent with PRKCA, PRKCD, PRKCH, PRKCI and $P R K C E$ ( $\mathrm{PKC} \alpha, \delta, \eta, \lambda / \mathrm{l}$ and $\varepsilon$ ) being expressed in most donors. Evidently, many $\mathrm{PKC}$ isoforms may play a role in osteogenesis of hMSCs and treatment of hMSCs with compounds that modulate PKC without isoform specificity will result in the activation or inhibition of several PKC isoforms within the same cell. This will result in a complex net effect on cell signaling and biological responses. Treatment of hMSCs with a broad blocker of PKC, calphostin C, displayed a dose-dependent inhibition of ALP expression in hMSCs, suggesting an essential role for PKC activity in maintaining or inducing ALP expression. This is in line with the proposed role of PKC $\delta$ in osteoblasts differentiation. In MC3T3 cells, PKC $\delta$ activates Runx2 [99, 140], and PKC $\delta$ mutant mice display reduced bone formation in the early embryonic skeleton due to impeding the onset of $O s x$ (osteorix) expression [100]. We used rottlerin to inhibit PKC $\delta$, and 
found that ALP expression was blocked completely in all circumstances, suggesting that $\mathrm{PKC} \delta$ is pivotal in maintaining the ALP expression of hMSCs. A note of care for this conclusion, which generally applies to the use of all pharmaceuticals in this manuscript, is that rottlerin may have biological effects on the activity of other proteins as well. Genetic interference in PKC $\delta$ activity is needed to conclusively demonstrate a role of PKC $\delta$ in hMSC osteogenesis.

Activation of the conventional and novel PKC isoforms using PMA yielded a complex response. Overall, PMA and other DAG-mimicking compounds had an inhibitory effect on ALP, mineralization and proliferation. Consistently, it was reported that $1 \mu \mathrm{M}$ PMA decreased ALP activity in the human osteosarcoma cell line UMR106 [141]. However, the inhibitory effect reached a maximum at intermediate levels of PMA, whereas at high concentrations of PMA, the inhibition was milder. The biphasic effect of PMA on hMSCs could be explained by assuming that the activation profile of PMA differs between different PKC isoforms and that different isoforms have opposing effects on osteogenesis. Hypothetically, intermediate concentrations of PMA could activate an anti-osteogenic PKC isoform but not affect a proosteogenic isoform. At high concentrations of PMA, the pro-osteogenic PKC is activated and counteracts the anti-osteogenic PKCs. Supporting this line of thought, we identified the conventional PKC family plus $\mathrm{PKC} \mu$ as negative regulators of osteogenesis using the inhibitor Gö6976. Upon treatment, we observed enhanced ALP expression, mineralization and collagen (I) expression in hMSCs. Thus, we have identified PKC isoforms with either a positive (PKC $\delta$ ) or negative role in hMSC osteogenesis. Similarly, it has been reported that overexpression of PKC $\alpha$ stimulates proliferation in NIH3T3 cells whereas overexpression of PKC $\delta$ is anti-proliferative [142]. In addition, Rossi and coworkers showed that 100nM PMA induced less PKC activity than 20nM PMA in haematopoietic precursors, and low PKC activity led these precursors into myelomonocytic differentiation, while high PKC activity favored eosinophil differentiation [143].

The proposed negative role of $\mathrm{PKC} \mu$ in osteogenesis is in contrast to what has been reported previously, where $\mathrm{PKC} \mu$ was shown to be involved in activating MC3T3 osteogenic differentiation [144, 145]. Furthermore, Cecil and colleagues concluded that PKC $\mu$ is implicated in BMP-2 and IGF-I induced RUNX2 activity in hMSCs and mineralization using a 10 $\mu \mathrm{M}$ dose of Gö6976 to demonstrate this [146]. However, it is known that Gö6976 has an IC50 on $\mathrm{PKC} \mu$ activity in the $\mathrm{nM}$ range and we found that $1 \mu \mathrm{M}$ Gö6976 leads to cell 
death. Furthermore, we demonstrate that 100nM Gö6976 enhanced osteogenic differentiation of hMSCs. The discrepancy cannot be explained by donor variability because we have repeated all the experiments in this manuscript with hMSCs from several donors and we report quantitative differences in response rather than qualitative. The experiments in the manuscript of Cecil et al. have been performed using commercially available hMSCs from one donor only. The medium used to expand and differentiate the cells contains a 10-fold higher concentration of dexamethasone than our medium $\left(10^{-7} \mathrm{M}\right.$ versus $10^{-8} \mathrm{M}$ respectively) and its chemical composition is not further disclosed by the supplier.

In conclusion, using a pharmaceutical approach we have identified that inhibition of the conventional PKC/PKC $\mu$ isoforms using Gö6976 stimulates osteogenic differentiation, whereas $\mathrm{PKC} \delta$ seemed to play a positive role in maintaining the osteogenic differentiation potential of hMSCs. From the bone tissue engineering point of view, either inhibition of conventional $\mathrm{PKC} / \mathrm{PKC} \mu$ or activation of $\mathrm{PKC} \delta$ in hMSCs is likely to benefit bone tissue engineering, which we are currently testing.

\section{ACKNOWLEDGEMENTS}

We wish to thank drs. L. Creemers and W. Dhert from the Department of Orthopaedics, UMC Utrecht and Dr. A. Renard from the Medical Spectrum Twente hospital for their generous supply of bone marrow aspirates. We would also thank R. I. Ravestein-van Os and R. H. L. van de Wetering from the Department of Target Discovery, Schering-Plough Research Institute for performing the microarray tests. The research is sponsored by a grant from Senter/Novem. 



\section{Chapter 3}

\section{In vitro and in vivo bioluminescent imaging of hypoxia in tissue engineered grafts}

Jun Liu ${ }^{1}$, Ana Barradas ${ }^{1}$, Hugo Fernandes ${ }^{1}$, Frank Janssen ${ }^{1}$, Bernke Papenburg $^{2}$, Dimitrios Stamatialis ${ }^{2}$, Anton Martens ${ }^{3}$, Clemens van Blitterswijk $^{1}$, Jan de Boer ${ }^{1}$

1. MIRA Research Institute, Department of Tissue Regeneration, University of Twente, 7500 AE, Enschede, The Netherlands.

2. MIRA Research Institute, Membrane Technology group, University of Twente, $7500 \mathrm{AE}$, Enschede, The Netherlands.

3. Department of Immunology, University Medical Center, 3508 AB, Utrecht, The Netherlands 


\section{ABSTRACT}

Survival and growth of cellular grafts in tissue engineering (TE) are limited by the rate of oxygen and nutrient diffusion. As such, monitoring the levels of nutrients and oxygen available to the cells is essential to assess the physiology of the cells and to evaluate strategies aiming at improving nutrient availability. In this manuscript, a reporter system containing the luciferase gene driven by a hypoxia responsive promoter was used to monitor cellular hypoxia in a tissue engineering context. We report that luciferase activity correlates with the oxygen tension in cell culture medium. When transgenic cells were seeded onto scaffolds and implanted in immune-deficient mice subcutaneously, luciferase activity was detected. To validate the response to oxygen levels of this reporter system, we cultured transgenic cells on biomaterials in a flow perfusion bioreactor and observed that cells in the bioreactor displayed a drastically lower luciferase activity compared to conventional static culture, and that higher luciferase activity is observed in the interior of a tissue engineered construct, illustrating the uneven oxygen distribution in $3 \mathrm{D}$ constructs under conventional static culture. We conclude that this reporter system is a versatile tool to investigate cellular oxygen availability in tissue engineering both in vitro and in vivo.

\section{INTRODUCTION}

A major issue in tissue engineering is to provide sufficient nutrients to maintain cell survival and growth in vitro and in vivo. The rate of diffusion limits the size of avascularised tissue to $100-200 \mu \mathrm{m}$ [147]. As a consequence, lack of nutrients results in central necrosis in tissue engineered grafts with a clinically relevant size $(>\mathrm{mm})$, thus leading to only limited tissue formation $[148,149]$. As a rule of thumb, cells can only survive up to $200 \mu \mathrm{m}$ from the periphery of a tissue engineered construct in vitro [41, 150]. Thus, it is still a challenge to grow large grafts both in vitro and in vivo. Current strategies to improve nutrient availability include improving diffusion by controlling pore size and porosity of scaffolds, by using flow perfusion bioreactors, and by stimulating vascular ingrowth using growth factors (e.g. VEGF and bFGF) and approaches to pre-vascularize the grafts [47]. For instance, it has been reported 
that ingrowth of vessels is faster in scaffolds with pores of $250 \mu \mathrm{m}$ than in scaffolds with smaller pores [48]. Similarly, the convective flow of perfusion bioreactors enhances the transport of oxygen and nutrients to the interior of the construct beyond the limits of diffusion [49], leading to improved cell viability in the interior and more homogeneous cell distribution in the scaffold [151]. Finally, Levenberg et al. demonstrated that a prevascular network of endothelial cells improved skeletal muscle tissue survival after implantation in vivo [55, 152]. Despite these positive results, there is no efficient method to evaluate oxygen availability in tissue engineered grafts in vitro or in vivo.

The firefly luciferase enzyme is widely used for molecular imaging, because it is able to report on many cellular processes, depending on the promoter used to drive luciferase gene expression [153, 154]. Luciferase oxidizes its substrate, luciferin, resulting in the emission of light with a broad emission spectrum and a peak at $\approx 560 \mathrm{~nm}$. This technique is widely used in molecular biology to investigate molecular signaling pathways, protein stability etc. [155], but it can also be used for non-invasive and real time imaging in small animals [77].

Among the nutrients essential for cell survival, oxygen seems to be particularly important, due to its high consumption rate, low solubility in culture medium and relatively slow diffusion rate [44]. Because oxygen is likely to be a rate limiting nutrient, it may act as a model molecule to reflect the cell's nutrient availability. With this aim, we investigated whether a hypoxia responsive element-luciferase (HRE-Luc) construct could be used to investigate $\mathrm{O}_{2}$ availability in tissue engineering. The HRE promoter construct is hypoxia inducible factor 1 (HIF1) dependent and thus the luciferase activity reflects HIF1 activity. HIF1 is a heterodimer containing an $\alpha$ subunit (HIF1 $\alpha$ ) and a $\beta$ subunit (HIF1 $\beta$ ), and its activity is regulated through HIF1 $\alpha$ stability (see ref [156] for a comprehensive review on HIF1). In normoxia (normal $\mathrm{O}_{2}$ tension), HIF1 $\alpha$ protein is degraded due to hydroxylation by prolyl hydroxylase and subsequent ubiquitination, resulting in degradation in the proteosome. During hypoxia, oxygen-dependent hydroxylation is inhibited, HIF $1 \alpha$ accumulates and translocates to the nucleus where it binds to the constitutively expressed HIF $1 \beta$ protein. Subsequently, the HIF1 heterodimer docks to HREs in target genes and activates them. Thus, HIF1 and its downstream target genes are regulated directly by $\mathrm{O}_{2}$ tensions.

The HRE-Luc construct was previously used to investigate the role of hypoxic stress in tumor development [80, 157] and wound repair [158]. It was 
reported that HIF1 plays a central role in tumor progression, invasion, and metastasis and some therapeutic strategies aim at targeting HIF1. Harada et al. evaluated the effect of a hypoxia-targeting prodrug (TOP3) on tumor development by monitoring the light intensity from tumor xenograft of HeLa/5HRE-Luc cells [159]. This construct was also used to screen inhibitors of hypoxia in cancer therapy [160]. In addition, the HRE-Luc reporter displayed very high luciferase activity from ischemic wounds in both rat and rabbit models [158]. In this study we assessed the possibility to use the HRE-Luc reporter to investigate hypoxia in cell-based tissue engineering both in vitro and in vivo.

\section{MATERIALS AND METHODS}

\section{Plasmid DNA}

The pGL3\HRE-Luc plasmid was kindly provided by Dr. Shinae Kizaka Kondoh (Kyoto University, Kyoto, Japan) [161, 162]. pCDNA3\HRELuc2 was generated by cloning the XhoI/HindIII HRE cassette from pGL3\HRE-Luc, and the HindIII/XbaI luciferase2 fragment from pGL4 (Promega) into pCDNA3 (Promega) after excision of the CMV promoter. The pCDNA3\CMV-Luc2 construct was created by cloning the XhoI/XbaI luciferase-2 fragment from pGL4 into pCDNA3.

\section{Transfection of the reporter gene and cloning of stable transformants}

The HRE-Luc transfected Chinese hamster ovary (CHO) A4-4 cell line was kindly provided by Dr. Kiyoshi Nose (Showa University, Tokyo, Japan), and we refer to it as HL cells. HL2 and CL2 cells were generated by transfecting CHO cells (CCL-61, ATTC) with pCDNA3\HRE-Luc2 or pCDNA3\CMV-Luc2 respectively, using Fugene 6 (Roche). Transfected cells were selected with $3 \mathrm{mg} / \mathrm{ml} \mathrm{G} 418$ (Sigma) for a week, and the resistant colonies were isolated and tested for luciferase activity. The cytomegalovirus promoter (CMV) is constitutively active, and light from CL2 cells will report cell number.

\section{Cell culture}

CHO cells were cultured in $\alpha$-MEM medium (Life Technologies) supplemented with 10\% fetal bovine serum (FBS, Cambrex), 2mM L-glutamine

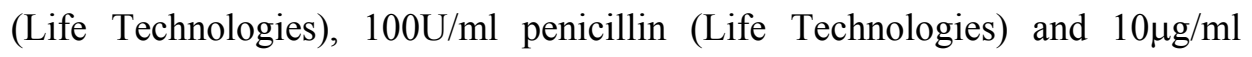


streptomycin (Life Technologies). Cells were grown at $37^{\circ} \mathrm{C}$ in a humid atmosphere with $5 \% \mathrm{CO}_{2}$. Medium was refreshed twice a week.

\section{Luciferase and Cyquant assay}

All samples were at least triplicate for luciferase and DNA quantification. Cells were washed with PBS 3 times and the luciferase activity was analyzed according to the manufacturer's protocol of the luciferase assay kit (Promega). The luminescence was detected by a Bio-orbit 1253 Luminometer (ABOATOX). Cell numbers were determined using the Cyquant DNA quantification kit (Invitrogen). Average luciferase activity was calculated by normalizing luciferase activity to the DNA amount.

To test deferoxamine (Sigma-Aldrich) effects, 20,000 HL cells per well were seeded in a 96-well plate, and deferoxamine was added to the medium for 24 hours in triplicate. At the end of this experiment, luciferase activity and cell numbers were determined using luciferase assay and Cyquant DNA amount assay.

To test the effect of cell density on the $\mathrm{O}_{2}$ level in the medium, $\mathrm{HL}$ cells were seeded at different densities in wells of a BD oxygen sensor plate (BD Bioscience) and in a normal 96-well plate in triplicate. After 24 hours the plates were applied for either quantifying $\mathrm{O}_{2}$ levels or used for determining luciferase expression.

\section{Bioluminescent imaging of cells in vitro}

HL and CL2 cells were seeded at the same densities as above in a 96well plate. Twenty-four hours later, cells were washed with PBS, supplied with $50 \mu 1$ luciferin (Promega) dilution (1:10 in PBS) per well, and bioluminescent imaging was performed using a CCCD camera (Biospace, Paris, France).

One percent agarose gels (Invitrogen) were mixed with 1 million HL cells and processed into cylinders with a diameter of $\varnothing 4.8 \mathrm{~mm}$. After one day, cells were examined for viability by a LIVE/DEAD Viab Cyto Kit (Invitrogen). Next, the gels were used to perform BLI by incubating the gels in a luciferin (Promega) solution (1:10 in PBS). The bioluminescence was recorded in realtime using a CCCD camera (LN/CCD-1300EB) with a 50-mm F1.2 Nikon lens (Roper Scientific), controlled by Metavue software (Universal imaging Corp., West Chester, PA, USA).

\section{In vivo imaging of luciferase activity in subcutaneous implants}


Two hundred thousand or one million cells in $100 \mu \mathrm{l}$ medium from both the HL2 and CL2 cell lines were seeded onto $3 \beta$-calcium phosphate ceramic particles (BCP) of $2-3 \mathrm{~mm}$ in size (kindly provided by Dr. Huipin Yuan, University of Twente, the Netherlands) as one sample. Four hours later, medium was added to each sample. Next day samples were implanted subcutaneously in 3 male nude mice (Hsd-cpb:NMRI-nu; Harlan) as previously described [28]. Meanwhile 3 samples were used to quantify the cell number and luciferase activity prior to implantation. Bioluminescent imaging was performed after intraperitonial injection of $100 \mu \mathrm{l}$ D-luciferin $(25 \mathrm{mg} / \mathrm{ml}$, Synchem Chemie, Kassel, Germany) using a CCCD camera (Biospace, Paris, France). All experiments were approved by the local Ethical Committee for Animal Experimentation and in compliance with the Institutional Guidelines on the use of laboratory animals.

\section{Dynamic culturing in bioreactors}

Two hundred thousand HL cells were seeded per 3 BCP particles. Cells were allowed to attach overnight, and 450 particles were transferred into a bioreactor with either $20 \%$ or $5 \% \mathrm{O}_{2}$ inlet. A direct perfusion flow bioreactor was used as described previously $[28,163]$. The medium flow rate was kept at $4 \mathrm{ml} / \mathrm{min}$. As a control 3 particles were transferred to each well of a non-tissue culture treated 25 -well plate at normoxia using $2 \mathrm{ml}$ of medium for 3 particles in all conditions. Medium was refreshed at day 2. At day 2 and 4, cell viability was evaluated using a Live/dead viability kit (Invitrogen), and 9 particles were sampled to measure luciferase activity and DNA amount.

\section{PLLA stacking experiment}

Twenty thousand cells were seeded overnight onto a round poly(Llactic acid) sheet (PLLA, [164]) with an area of $1.8 \mathrm{~cm}^{2}$. Four sheets were stacked into a well of a 24-well plate with cells on top of the lower 3 sheets. The sheets were separated by $1 \mathrm{~mm}$ thick rubber $\mathrm{O}$ rings and $2 \mathrm{ml}$ of medium was used per well. A 24-well plate with the same amount of cells but without PLLA sheets was used as a control. After 8 hours, 1 day and 2 days, cell viability was evaluated and luciferase activity was determined.

\section{Statistical analysis}

Statistical analyses were performed using Student's t-test or one-way ANOVA. ${ }^{*} p<0.05, * * p<0.01, * * * p<0.001$. 


\section{RESULTS}

\section{Luciferase activity driven by a HIF1-dependent promoter reflects $\mathrm{O}_{2}$ availability}

We used a reporter system basing on the luciferase gene under the control of HIF1-dependent sequences (Fig. 1A). Deferoxamine (DFO) treatment is known to induce the accumulation of HIF1 [165]. Indeed, when we incubated HL cells with DFO for one day we observed a dose-dependent increase in luciferase activity (Fig. 1B). Next, we analyzed the effect of cell density on the $\mathrm{O}_{2}$ level in culture medium. One day after cell seeding, the $\mathrm{O}_{2}$ level in the medium dropped in wells with high numbers of cells (Fig. 1C). In parallel, luciferase expression in $\mathrm{HL}$ cells correlated with the $\mathrm{O}_{2}$ level in the medium (Fig. 1D). In agreement with this, using BLI, we detected increased light intensity at a cell density of $4 \times 10^{4} \mathrm{HL}$ cells or more, while the light intensity from CL2 cells was dependent on cell numbers (Fig. 1E). Thus, the hypoxia reporter reflects the cellular HIF1 activity which in its turn reflects the $\mathrm{O}_{2}$ level available to cells. We also monitored $\mathrm{O}_{2}$ availability in a tissue engineered 3D gel construct (Fig. 1F). One million HL cells were incubated in a cylinder of agarose gel. After one day, the cells were alive but they expressed luciferase by emitting strong light, indicating the oxygen deficiency in such culturing conditions.

\section{Monitoring cell survival and cellular nutrient status in vivo using luciferase}

For bone tissue engineering, we typically use $2-3 \mathrm{~mm}$ porous ceramic scaffolds to graft cells. The absence of blood vessels in vivo most likely represents an ischemic environment where the lack of nutrients limits tissue formation. In order to monitor cell survival and nutritional status in vivo, we implanted CL2 and HL2 cells in immune-deficient mice. Either $2 \times 10^{5}$ (C1, H1) or $10 \times 10^{5}(\mathrm{C} 2, \mathrm{H} 2)$ cells per 3 porous ceramic particles were seeded for one day, and $2-3 \times 10^{5}(\mathrm{C} 1, \mathrm{H} 1)$ and $6 \times 10^{5}(\mathrm{C} 2, \mathrm{H} 2)$ cells were quantified on the scaffolds prior to implantation (Fig. 2A). High luciferase activity was observed from samples seeded with high numbers of HL2 cells using the biochemical luciferease assay (H2 in Fig. 2A). After implantation, we used bioluminescent imaging to detect the luciferase from all 4 samples and as expected, a higher signal intensity was observed from implants with high cell numbers (Fig. 2B and $\mathrm{C}$ ). We also detected that the absolute signal intensity from HL2 cell implants increased from day 0 to day 2 , which indicates a more pronounced 
A

5xHRE VEGF fragment

E1b minimal promoter
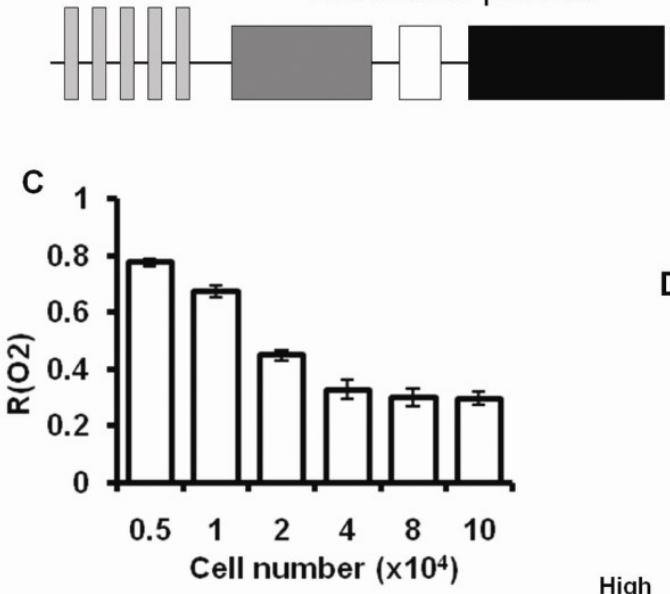

E

HRE-Luc

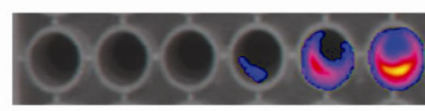

CMV-Luc2

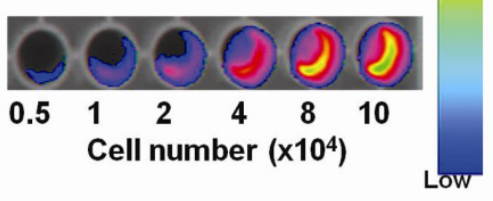

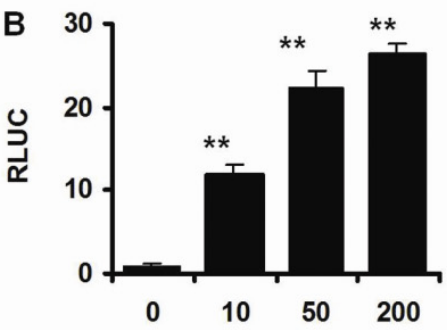

Deferoxamine $(\mu \mathrm{M})$

D

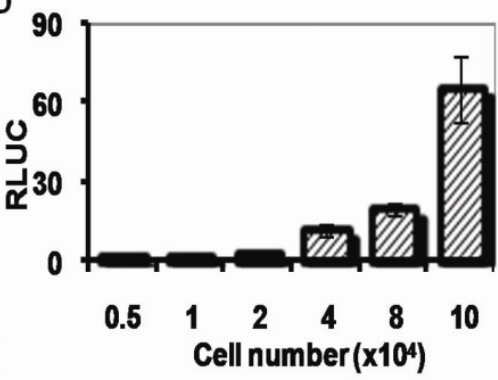

F

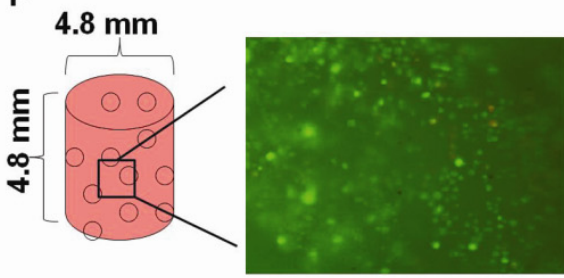

Live/dead staining

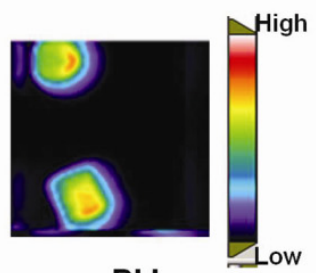

BLI

Figure 1. HL cells respond to hypoxia by inducing luciferase activity. (A) The HRE-Luc construct comprising the luciferase reporter gene (Luc), a fragment of the VEGF promoter (VEGF fragment) and an E1b minimal promoter sequence [161]. (B) Deferoxamine enhanced luciferase activity dose-dependently. The relative luciferase activity (RLUC) is the ratio of the DNA-normalized luciferase activity of each sample to the control sample $\left(0 \mu \mathrm{M}\right.$ deferoxamine). (C) Relative oxygen availability $\left(\mathrm{RO}_{2}\right)$ is expressed as the level of oxygen relative to that in medium without culturing HL cells $(=1)$. (B) The luciferase activity of HRE-Luc cells was quantified by using a biochemistry assay. The relative luciferase activity (RLUC) is the ratio of the DNA-normalized luciferase activity at different cell densities to that at the lowest cell density. (C) Bioluminescent imaging of a 96-well plate with different cell numbers one day after seeding at the indicated density. Pseudo-coloring represents the light intensity. (D) HRE-Luc cells were cultured in a $1 \%$ agarose gel cylinder with a dimension of $4.8 \mathrm{~mm}$. The green staining indicated that almost all cells were viable. Values are mean $\pm \mathrm{SD}$ (standard deviation, $\mathrm{n}=3$ ). Statistical significance is calculated using a Student's t-test. $* * p<0.01$. 

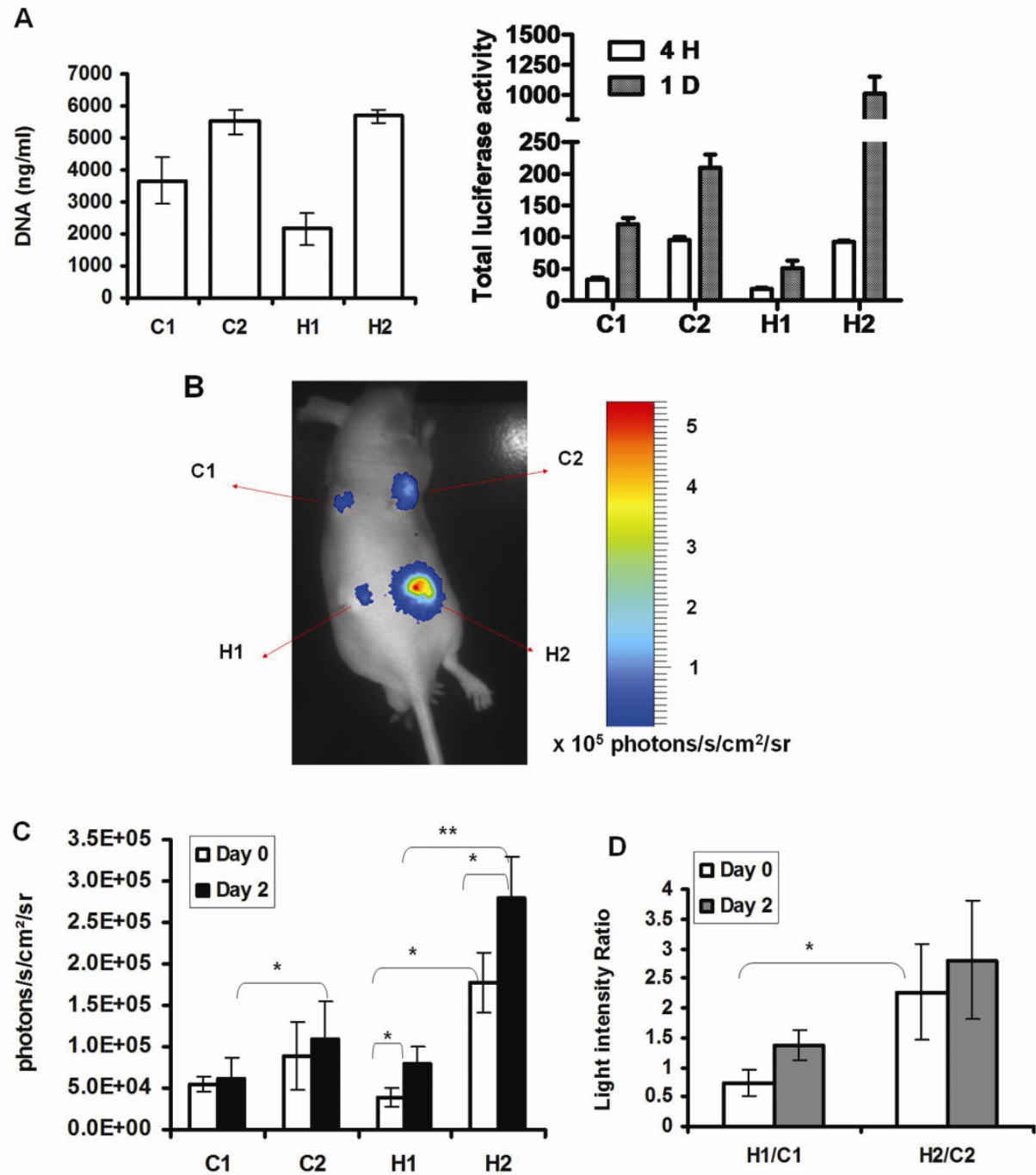

Figure 2. Bioluminescent imaging of hypoxia and cell survival in vivo. (A) The cell number and total luciferase activity were analyzed on the samples prior to implantation by DNA amount measurement and luciferase assay. $\mathrm{C} 1$ and $\mathrm{H} 1$ are CL2 and HL2 cells seeded at low cell density respectively; C2 and H2 are CL2 and HL2 cells seeded at high cell density respectively. (B) Bioluminescent imaging of CL2 and HL2 cell-seeded implants in vivo at day 2. Pseudo-coloring represents the light intensity. All mice ( $\mathrm{n}=3$ ) showed the same light intensity pattern. (C) Average light intensity from the 4 implants at day 0 and day 2 . The quantitative data were generated by averaging light intensities from 10-minute imaging after the light intensity reached a plateau after 20 minutes luciferin injection. (D) The ratio of light intensity from HL2 cells normalized to that from CL2 cells at day 0 and 2 revealed increased hypoxia on the scaffolds seeded with more cells and longer time implanted in vivo. Values are mean $\pm \mathrm{SD}$ (standard deviation, $\mathrm{n}=3$ ). Statistical significance is calculated using a two-tailed Student's t-test. * $p<0.05, * * p<0.01$.

oxygen deficiency for the grafted cells at day 2. HRE activity per cell was calculated by expressing the ratio of HRE activity to CMV activity at day 0 and 
day 2 (Fig. 2D). The $\mathrm{H} 1 / \mathrm{C} 1$ ratio increased from day 0 to 2 , and at day 0 , the $\mathrm{H} 2 / \mathrm{C} 2$ ratio was higher than the $\mathrm{H} 1 / \mathrm{C} 1$ ratio. Taken together, the HRE-Luc reporter system reflects the oxygen levels in the cell's environment in vitro and in vivo. To further demonstrate this, we tested its response to differential oxygen availability in two tissue engineering models.

\section{Monitoring hypoxia in a flow perfusion bioreactor}

It is well accepted that the conventional static culture of 3D cellular constructs induces a heterogeneous nutrient delivery, and flow perfusion bioreactors are used to optimize nutrient availability. We seeded HL cells onto porous ceramic particles and incubated them in flow perfusion bioreactors at two constant $\mathrm{O}_{2}$ concentrations of $20 \%$ and $5 \%$. Cells were alive as verified by live/dead staining (data not shown) and proliferated in both bioreactors indicated by the linear oxygen consumption during the 4-day culturing (Fig. 3A and for bioreactor with 5\% oxygen data not shown). In agreement with this, quantification of cell numbers showed that cells proliferated in all three conditions, and $20 \% \mathrm{O}_{2}$ supported a higher cell number than 5\% $\mathrm{O}_{2}$ at day 4 (Fig. 3B). Then, we determined HRE activity at day 2 and day 4. Cells grown in the $5 \% \mathrm{O}_{2}$ bioreactor displayed higher luciferase expression than cells grown in the $20 \% \mathrm{O}_{2}$ bioreactor at both time points (Fig. 3C). In parallel we compared the effect of static and dynamic culture on HRE activity and cell proliferation. Interestingly, cell proliferation in both static and dynamic conditions was similar but static culturing induced an 8 times higher luciferase expression than cells grown in the bioreactor with $20 \% \mathrm{O}_{2}$ (Fig. 3C).

\section{A nutrient gradient revealed by luciferase activity from $\mathrm{HL}$ cells}

Cells growing in the interior of a 3D graft are more deprived of nutrients than those growing on the periphery [150]. We simulated a 3D construct by stacking HL cell-seeded sheets of non-porous PLLA in a 24-well plate (Fig. 4A). The oxygen availability in each layer was then dependent on $\mathrm{O}_{2}$ diffusion through medium and polymer sheet, and also on the $\mathrm{O}_{2}$ consumption from other layers above, thus mimicking the real oxygen diffusion in 3D TE constructs. After 8 hours of culture, luciferase expression was measured, and it was similar for all layers (Fig. 4B). After 1 day, we observed that the average luciferase expression increased with increasing distance from the bulk medium, which was even more pronounced after 2 days. 

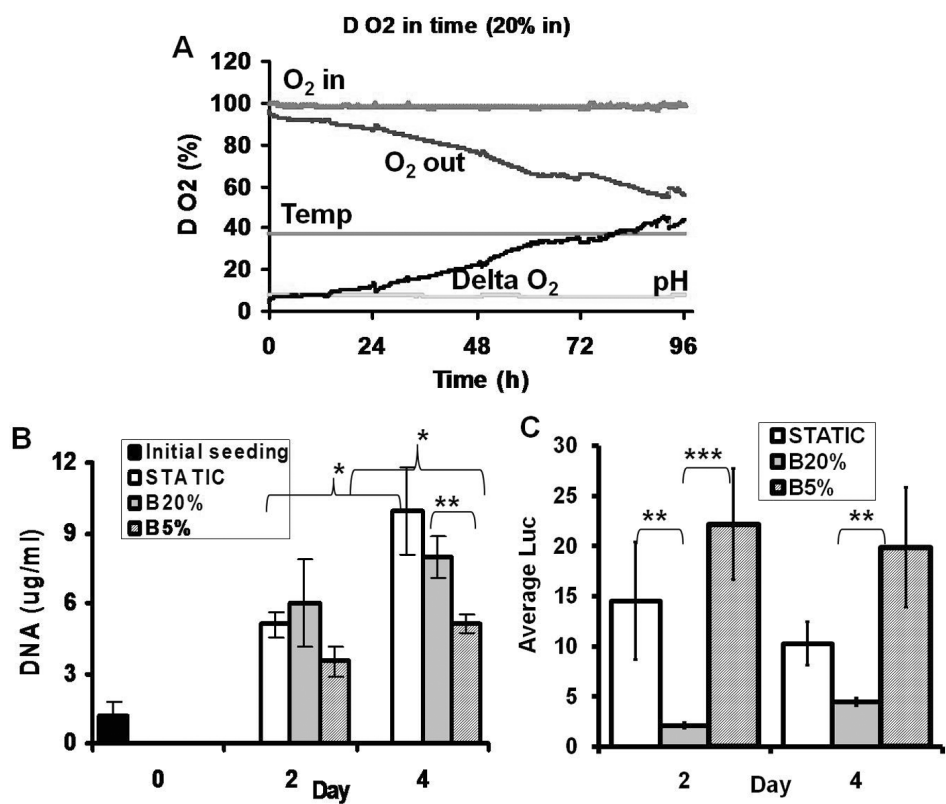

Figure 3. Hypoxia in static versus dynamic culturing. (A) Online monitoring of temperature, $\mathrm{pH}$, oxygen concentration at the inlet, at the outlet and the difference (delta $\mathrm{O}_{2}$ ) in a bioreactor with $20 \% \mathrm{O}_{2}$ inlet. (B) HL cell proliferation in different culturing conditions. Values are mean \pm SD (standard deviation, $n=3$ ). (C) Average Luc was the DNA-normalized luciferase activity of HL cells grown in either static culture or in bioreactors with $5 \%$ and $20 \% \mathrm{O}_{2}$ inlet (B5\% and B20\% respectively). Statistical significance is calculated relative to cells grown in bioreactor with $20 \% \mathrm{O}_{2}$ inlet using one-way ANOVA. ${ }^{*} p<0.05, * * p<0.01, * * *$ $p<0.001$.
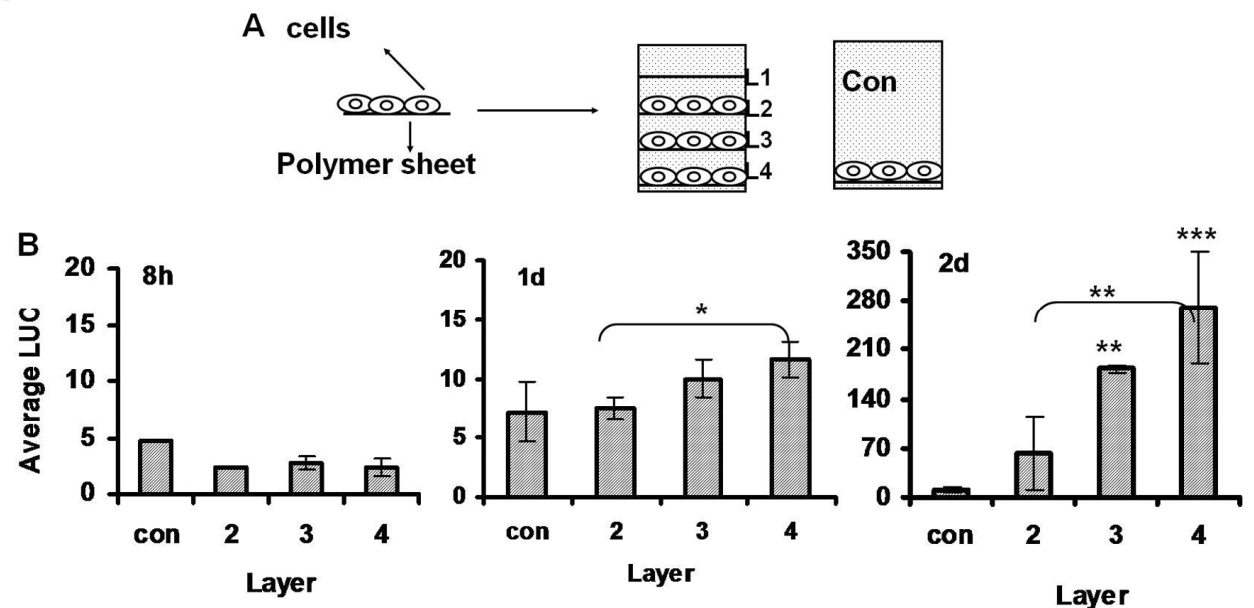

Figure 4. A nutrition gradient in a 3D construct. (A) Diagram representing stacks of PLLA sheets seeded with HL cells. L1 to L4 refer to the cell-seeded sheets. Con, control well. No dead cells were detected during the 4-day culturing in all layers as demonstrated by both live/dead staining and LDH measurement (data not shown). (B) Luciferase activity in HL cells at different time points and growing on the different layers. Average Luc was determined as described above. Values are mean \pm SD (standard deviation, $n=3$ ). Statistical significance is calculated relative to cells grown in control wells using one-way ANOVA. 


\section{DISCUSSION}

Non-invasive imaging of hypoxia in cell-based tissue engineering both in vitro and in vivo can be an important tool to monitor the cellular nutrient status. In this report, a HIF1-dependent promoter was used to drive luciferase expression. We demonstrated that luciferase activity correlated with HIF1 activity corresponding to low $\mathrm{O}_{2}$ tension in the medium by adjusting different cell seeding densities. In addition, controlling $\mathrm{O}_{2}$ inlet concentration resulted in an induction of luciferase activity, where the bioreactor with $5 \% \mathrm{O}_{2}$ had a much higher luciferase activity than the one with $20 \%$ oxygen in the perfused medium. Taken together, these observations validate the response of this reporter system to $\mathrm{O}_{2}$ level in the cell culturing environment. To further expand this system's application and to explore its sensitivity, two tissue engineering culture models were investigated. Perfusion bioreactors are used to improve the supply of oxygen and other nutrients to cells, but so far there is no direct proof for this. We observed a positive effect of flow perfusion culturing on oxygen availability, as shown by the higher luciferase activity in static culture while very low or hardly any luciferase activity from a bioreactor with $20 \% \mathrm{O}_{2}$. Previous reports describe that 3D constructs have a gradient of nutrients, with nutrient depletion in the inner part of the construct resulting in heterogenic cell growth $[45,166]$. HRE-Luc reporter activity could indeed monitor a lack of oxygen for cells growing on the deep layers of the stacked sheets. Besides being used in vitro, the reporter system was also used in vivo. Light intensity in Fig. 2 clearly reflected different cell numbers and their hypoxia levels. The strong light from HRE-Luc2 cells demonstrated that cells experienced severe nutrient deficiency indicating the necessity of instant nutrient or $\mathrm{O}_{2}$ supply after implantation in vivo. So far, we demonstrated that $\mathrm{O}_{2}$ can be used as an index for investigating cellular nutrient status, and the HRE-Luc reporter system could be a good tool to investigate $\mathrm{O}_{2}$ or even an index of nutrient availability and distribution in tissue engineering.

The conventional methods for monitoring $\mathrm{O}_{2}$ include micro-electrodes [150] or sensors in the medium [82, 163, 167] and immunostaining for pimonidazole [168] or HIF1 $\alpha$. Despite their wide applications, each has their drawbacks such as invasiveness, indirectness, high cost, inaccuracy and timeconsumption. Although we mostly used invasive, biochemical assays to measure luciferase activity in this report, the HRE reporter system can be used as a non-invasive, accurate, fast and direct tool for cellular $\mathrm{O}_{2}$ level and HIF1 
activity (for instance Fig. 1D versus 1E). It is important to note that cells grown at 4,8 and $10 \times 10^{4}$ cells/well display an equal relative oxygen level of $0.3(6 \%$ oxygen in the medium), whereas the HRE-luc signal increases, especially at $10 \times 10^{4}$ cells/well. It is reported that the activation of the hypoxia response, i.e. stabilization of HIF1 is exponentially related to hypoxia degree in HeLa cells, which starts at $3 \%$ and reaches a peak at $0.5 \%$ oxygen [169]. Apparently, in our experiment we have reached a lower level of oxygen than the level detected by the oxygen detection plate.

Besides providing physiological information, HRE-Luc and CMV-Luc reporter systems can be applied in evaluating methods to improve cell survival. For example, some reports showed improved cell survival by $\mathrm{O}_{2}$ releasing materials such as perfluorocarbon (PFC) $[170,171]$ or pretreatment with hypoxia before implantation [56]. This can be monitored using the HRE-Luc and CMV-Luc reporter systems dynamically in vivo.

Luciferase activity was quantified and expressed as the light intensity unit using both non-invasive imaging and biochemical assays. There are several factors determining the light intensity in one measurement including luciferase abundance per cell, cell number and luciferin concentration but also $\mathrm{O}_{2}$ and ATP availability for in vivo tests because $\mathrm{O}_{2}$ and ATP are co-factors in the luciferin reaction. If the factors mentioned are rate limiting, the luciferase activity measured is actually an underestimation. Inoue and colleagues found that the exposure to $\mathrm{O}_{2}$ was required for bioluminescent light emission by showing that the luminescence from tumor cell transplantation disappeared after the animal was killed but air injection restored light emission [172]. ATP was thought to be a key mediator in bioluminescence [173] due to the fact that luciferase-luciferin reaction is ATP-dependent [1], and it is known that hypoxia decreases cellular ATP production. In previous studies, we also noticed that luciferase activity is impaired in conditions of severe nutrient/oxygen deficiency indicated by a decline in luciferase activity (data not shown). Thus, the data present here were all collected under mild culturing conditions which was defined by measuring the glucose availability.

In conclusion, we demonstrate that the HRE-reporter system can be a useful tool to investigate cellular $\mathrm{O}_{2}$ or nutrient status in tissue engineered constructs non-invasively both in vitro and in vivo. We suggest that this reporter system can be used to investigate strategies to improve nutrient availability such as optimizing the scaffold architectures in tissue engineering. 


\section{ACKNOWLEDGEMENTS}

We wish to thank dr. Shinae Kizaka Kondoh from Kyoto University, Kyoto, Japan, and dr. Kiyoshi Nose from Showa University, Tokyo, Japan for providing the plasmid DNA with HRE-Luc and transfected Chinese hamster ovary cells respectively. We also thank Gerard Geelen and Agnes Goderie for excellent animal care. The research is sponsored by a grant from Senter/Novem. 


\section{Chapter 4 \\ Imaging nutrient diffusion in tissue engineered grafts}

\section{Jun Liu ${ }^{1}$, Janneke Hilderink ${ }^{1}$, Tom Groothuis ${ }^{2}$, Cees Otto ${ }^{2}$, Clemens van Blitterswijk ${ }^{1}$, Jan de Boer ${ }^{1}$}

1. MIRA Research Institute, Department of Tissue Regeneration, University of Twente, Enschede, The Netherlands

2. MIRA Research Institute, Biophysical Engineering Group, University of Twente, Enschede, The Netherlands 


\section{ABSTRACT}

Limited nutrient diffusion in three-dimensional constructs is a major concern in tissue engineering. Therefore, monitoring nutrient availability and diffusion within a scaffold is an important asset. In this study, we have investigated the diffusion of oxygen, luciferin and the sugar dextrane within tissue engineering constructs using optical imaging technology. Firstly, oxygen availability and diffusion was investigated using transgenic cell lines in which a hypoxia-responsive element drives either the luciferase or green fluorescent protein gene. We observed that cell density determines hypoxia level in hydrogels and oxygen limitation was observed in an agarose gel starting at $200 \mu \mathrm{m}$ from the periphery using confocal imaging. Diffusion of luciferin was monitored real-time in agarose gels using a cell line in which the luciferase gene was driven by a constitutively active CMV promoter. We found that gel concentration affected the diffusion rate of luciferin. Furthermore, we assessed the diffusion rates of fluorescent dextrane molecules of different molecular weights in biomaterials by evaluating the fluorescent recovery rate after photobleaching (FRAP). We observed that diffusion depended both on the molecular size and the gel concentration. In conclusion, we have identified a set of efficient tools to investigate molecular diffusion of a range of molecules, which can be valuable tools to optimize biomaterials design in order to improve nutrient delivery.

\section{INTRODUCTION}

Currently, clinical application of cell based tissue engineering is limited to tissue sheets or a-vascular tissues such as bladder, skin and cartilage because of restricted nutrient supply in larger 3D constructs both in vitro and in vivo. Nutrient diffusion in tissue is limited to a distance of $100-200 \mu \mathrm{m}[44,147]$. In tissue-engineered constructs, cell survival, proliferation and even differentiation largely depend on nutrient availability. It was reported that 3D tissue engineered constructs display a gradient of nutrient availability resulting in heterogenic cell growth in the graft $[45,166]$. Therefore, understanding the diffusion, distribution and availability of nutrient molecules and growth factors in cell/material constructs is necessary because it is a crucial step in tissue survival and formation. 
Due to its low solubility, oxygen is expected to be one of the first nutrients to become limiting and is therefore a critical nutrient in cell survival [42]. In contrast to the human body, tissue-engineered constructs lack natural vasculature and supply of oxygen can occur by molecular diffusion only [41]. Therefore, oxygen is recognized as a model nutrient. Unfortunately, it is difficult to monitor molecular diffusion gradients and to date only oxygen has been studied extensively by a number of techniques including electrodes [150], oxygen-quenched luminescence [166], phosphorescence quenching microscopy [174] and electron paramagnetic resonance [175]. On the other hand, very limited knowledge is available on the availability and diffusion of other nutrients or metabolites (e.g. glucose, lactic acid) in 3D grafts.

Previously, we and others have used transgenic cell lines, in which a hypoxia responsive element drives the luciferase gene to study oxygen levels in tissue engineering systems (chapter 3 ) or tumor research [80, 157]. The cells express luciferase in the case of limited oxygen availability, and luciferase activity can be evaluated by bioluminescent imaging or using an enzymatic assay. We showed that chinese hamster ovary cells harbouring this construct (HL cells) expressed luciferase when cultured at low oxygen conditions. Moreover, HL cells detected more oxygen deficiency when a 3D cell-seeded graft was grown in a conventional static culture, as compared to perfused bioreactors. In addition, when the HL-seeded grafts were implanted subcutaneously in nude mice, strong luciferase activity was observed, indicating a lack of nutrients in vivo. Thus, cells with this reporter system could serve as a non-invasive, quantitative and efficient tool to study oxygen availability [176].

Besides reporter-based cellular assays, it is also possible to monitor molecular diffusion. Fluorescence recovery after photobleaching (FRAP) has been used to investigate protein diffusion in the cell nucleus, cytoplasm and membranes [177, 178]. This approach, first described in 1976 by Axelrod and colleagues [179], involves irreversible photobleaching of fluorescently labeled molecules in a defined region of the specimen by a short and intense laser pulse. The recovery of fluorescence in this area, due to Brownian diffusion of unbleached molecules from other parts of the sample into the bleached region, is an indicator of the mobility of the fluorophores. The diffusion constant D, which is a measure of the rate of movement of the molecules in absence of flow or active transport, reflects the mean square displacement per unit of time (usually in $\mu \mathrm{m}^{2} \cdot \mathrm{s}^{-1}$ ) [180]. The diffusion constant for a particle in a free volume 
is described by the Stokes-Einstein formula: $D=\frac{k T}{6 \pi \eta R}$, where $\mathrm{T}$ is the absolute temperature, $\eta$ is the viscosity of the solution, $\mathrm{k}$ is the Boltzmann constant and $\mathrm{R}$ is the hydrodynamic radius of the particle. For a soluble spherical protein, an eightfold increase in size will lead to a twofold decrease in D [180, 181]. Pinte reported on the diffusion of polymer additives in food using FRAP, where he demonstrated size-dependent diffusion of food additives [182]. Leddy and colleagues demonstrated that molecular diffusion depended on molecular size and the zone of articular cartilage in which the molecules were present [183].

In this study, we have adopted both cell reporter and FRAP technology to investigate molecular diffusion and availability of oxygen, proteins and sugars in tissue engineered 3D constructs.

\section{MATERIALS AND METHODS}

\section{Cell culture}

Chinese hamster ovary (CHO) cells (CCL-61, ATTC) were cultured in $\alpha$-MEM medium (Life Technology) supplemented with $10 \%$ fetal bovine serum (FBS, Cambrex), 2mM L-glutamine (Life Technologies), 100U/ml penicillin (Life Technologies) and $10 \mu \mathrm{g} / \mathrm{ml}$ streptomycin (Life Technologies). Cells were grown at $37^{\circ} \mathrm{C}$ in a humid atmosphere with $5 \% \mathrm{CO}_{2}$. Medium was refreshed twice a week.

\section{Plasmid DNA}

The pHRE-LUC plasmid was kindly provided by Dr. Kiyoshi Nose (Showa University, Tokyo, Japan) $[161,162]$. To create pCDNA3\HRE-GFP, a HindIII/XbaI GFP fragment and the HRE fragment were inserted into pCDNA3 from which the CMV promoter was removed. To create pCDNA3\CMV-Luc2, Luc2 was excised from pGL4 and inserted into pCDNA3.

\section{Transfection of the reporter gene and cloning of stable transformants}

The HRE-Luc transfected CHO cell line A4-4 was kindly provided by Dr. Kiyoshi Nose (Showa University, Tokyo, Japan). We refer to it as HL cells in this manuscript. CHO cells were transfected with both pCDNA3\HRE-GFP and pCDNA3\CMV-Luc2 using Fugene 6 agent (Roche), and subsequently cultured in medium with $3 \mathrm{mg} / \mathrm{ml} \mathrm{G} 418$ (Sigma) for a week. Antibiotic-resistant 
cells were seeded at very low density, and colonies were isolated and tested for luciferase activity.

\section{Luciferase and Cyquant assay}

Luciferase activity and DNA quantification was assessed in at least triplicate. Cells were 3 times washed with PBS and lysed with the lysis buffer provided in the luciferase assay kit (Promega). $20 \mu \mathrm{l}$ of lysate was added to $100 \mu \mathrm{l}$ of luciferin substrate and luminescence was detected using a Bio-orbit 1253 Luminometer (ABOATOX). Cell numbers were determined using the Cyquant DNA quantification kit (Invitrogen) with $50 \mu$ l of cell lysate according to the manufacturer's protocol. Average luciferase activity was calculated by normalizing luciferase activity to the DNA amount.

\section{Viability staining}

A staining solution was made of $6 \mu \mathrm{M}$ ethidium homodimer and $1 \mu \mathrm{M}$ calcein (Invitrogen). Cells were washed in PBS and subsequently stained by covering the samples with staining solution and incubating for 15 minutes at $37^{\circ} \mathrm{C}$ in the dark. Samples were visualized by fluorescence microscopy (Nikon Eclipse E600).

\section{Real-time imaging of the luciferase activity in $3 D$ gel constructs}

Matrigel (BD Bioscience) and agarose gels (Invitrogen) with various concentrations were mixed with HRE-Luc (HL) cells and CMV-Luc2 (CL2) cells, and were processed into cylinders with a dimension of $\varnothing 4.8 \mathrm{~mm}$. After one day, cells were examined for viability as mentioned above. Then, gels were used to perform bioluminescent imaging by incubating the gels in a luciferin solution. The bioluminescence was recorded in real-time using a CCCD camera (LN/CCD-1300EB) with a 50-mm F1.2 Nikon lens (Roper Scientific), controlled by Metavue software (Universal imaging Corp., West Chester, PA, USA). For each image, photons were collected for one minute. Quantification of light intensity was performed using Image $\mathrm{J}$ software.

\section{Fluorescent imaging of $3 \mathrm{D}$ gel constructs}

HRE-GFP CHO cells were incubated with agarose gel using the same procedure as described above. A viability assay was performed and the remaining gel slices were fixed in $10 \%$ formalin. The slides were stained with DAPI (DAKO) to stain the cell nuclei. The GFP expression of the cells was analyzed by confocal fluorescence microscopy, using a 10x objective (BD 
pathway 435). The acquired images were analyzed using Attovision software. This experiment was performed in duplicate or triplicate with one image from each gel sample.

\section{Diffusion measurements}

Stock solutions of fluorescein isothiocyanate (FITC, 389Da) and three fluorescein-labeled dextrans (3, 10 and $70 \mathrm{kDa}$, Invitrogen) were prepared by dissolving in Tris-EDTA buffer. Agarose gels were prepared and before gelation, the viscous agarose was mixed with the dye solution (final concentrations dye: $5 \mu \mathrm{M}$ and $50 \mu \mathrm{M}$, final concentrations agarose: $0.5,1$ and $2 \%$ ) and cooled to room temperature to solidify. The gel was cut in slices of approximately $1 \times 4.8 \times 4.8 \mathrm{~mm}$.

Table 1. Size comparison of dextrans studied and relevant molecules (see ref [183]).

\begin{tabular}{c|c}
\hline Molecule & Molecular weight (kDa) \\
\hline Dextran-70 & 70 \\
TGF-b & 25 \\
BMP-2 & 18 \\
Dextran-10 & 10 \\
IGF & 7.6 \\
Insulin & 5 \\
PTH (1-34) & 4.1 \\
Dextran-3 & 3 \\
Glucose & 0.18 \\
FITC & 0.389 \\
Luciferin & 0.28 \\
Oxygen & 0.032 \\
\hline
\end{tabular}

FRAP experiments were performed on 5 slices per condition, using a laser scanning confocal microscope (Zeiss LSM 510) with a 40x objective. The images $(512 \times 512$ pixels) with a resolution of $0.44 \mu \mathrm{m} /$ pixel were taken in an optical slice of less than $6.3 \mu \mathrm{m}$. A $488 \mathrm{~nm}$ laser line was used for illumination and the fluorescence was detected with a longpass filter at $505 \mathrm{~nm}$. Bleaching was performed on a circular region $(\mathrm{r}=22$ or $32 \mu \mathrm{m})$ at $100 \%$ laser intensity, during 150 iterations. Dried samples were used as a positive control to determine the minimal number of iterations needed to completely bleach the area. The average intensity of the bleached area is normalized to the intensity in an unbleached part of the sample to correct for background bleaching, and fractional fluorescence recovery is plotted as: $f_{k}(t)=\frac{f_{R O I}(t) / f_{r e f}(t)-f_{R O I}(0) / f_{r e f}(0)}{f_{R O I}(\infty) / f_{r e f}(\infty)-f_{R O I}(0) / f_{r e f}(0)}$, with $\mathrm{f}_{\mathrm{ROI}}$ the intensity in the bleached 
spot and $\mathrm{f}_{\mathrm{ref}}$ the intensity in a region outside the ROI, at time $\mathrm{t}$, immediately after bleaching $(\mathrm{t}=0)$ and after complete recovery $(\mathrm{t}=\infty)$. The recovery curve was fitted (GraphPad Prism 5.0) to a one-phase association curve fit: $f(t)=f_{0}+$ $\left(f_{\text {plateau }}-f_{0}\right) *\left(1-e^{-\frac{1}{\tau} t}\right)$, and half recovery times were obtained from this curve fit by $T_{1 / 2}=\ln 2 * \tau . \mathrm{R}^{2}$ values ( 1 for perfect fit) of the curve fit were determined. Diffusion coefficients, which are proportional to the rate of fluorescence recovery and the size of the photobleached area, were calculated by $D=\gamma_{D} \frac{\omega^{2}}{4 \tau_{1 / 2}}$, with $\omega$ the radius of the ROI disc and $\gamma_{\mathrm{D}}$ a constant depending upon beam shape, type of transport and bleaching parameter $\mathrm{K}$, which expresses the amount of bleaching induced in time [179]. Since these parameters are the same for all conditions in these experiments, $\gamma_{D}$ was taken as 1 . This analysis assumes a two-dimensional configuration. Since the images are recorded in a thin section of the sample (optical slice $<6.3 \mu \mathrm{m}$ ), diffusion in and out of the imaging plane at the third dimension can be neglected.

Statistical analysis

Statistical analyses were performed using one-way or two-way ANOVA. $* p<0.05, * * p<0.01, * * * p<0.001$.

\section{RESULTS}

\section{Oxygen limitation in a $3 D$ gel construct}

We previously demonstrated that the HRE-Luc reporter system can be used to detect hypoxia availability in tissue engineered constructs [176]. Luciferase can be quantified in terms of light emission in the presence of its substrate luciferin. Here, we further applied this technology to investigate oxygen availability in 3D gel constructs. 1 million HL cells were seeded in cylinder-like $1 \%$ agarose gels with a diameter of $4.8 \mathrm{~mm}$. The gels were incubated at normoxia, and were performed with live/dead staining to check the viability: on day 1 , all cells were stained green indicating viable, while on day 2 , cells died in most of the construct except those at periphery (data not shown). Then, BLI was performed on the gels at day 1 (Fig. 1A). Light emission was detected from the gels using BLI, revealing that the oxygen level was so low that a hypoxic response had started. As observed before for HL cells grown in $2 \mathrm{D}$, the maximum light intensity per sample depended on the number of cells in 
the gel (Fig. 1B). The higher the cell concentration, the higher level of hypoxia was. Because the light emission was monitored using a CCCD camera, which is a non-invasive technique, it was possible to plot the light intensity from the gels over time (Fig. 1A). We observed a gradual increase in light intensity from gels during imaging. The light intensity also depended on the luciferin concentration, but the dynamic development was same in both luciferin concentrations with a plateau after about 60 minutes. The gradual increase in light intensity over this period most likely reflects diffusion of luciferin into the gel.

Diffusion properties depend on the material used and we assumed that a high concentration of agarose limits oxygen diffusion more than a low concentration of agarose. To demonstrate this, we performed bioluminescent imaging on agarose gels with 1 million HL cells at 3 different concentrations $(0.5,1$ and $1.2 \%$ agarose $\mathrm{w} / \mathrm{v})$ after 1 day culturing in normoxic incubators. To our surprise, we observed a higher plateau of light intensity in the lower percentage gels (Fig. 2A and B). We noticed that the slopes of these curves differed, which reflects the respective luciferin diffusion rates. Thus, the reverse relationship between bioluminescence and gel concentration was likely due to limited luciferin diffusion.
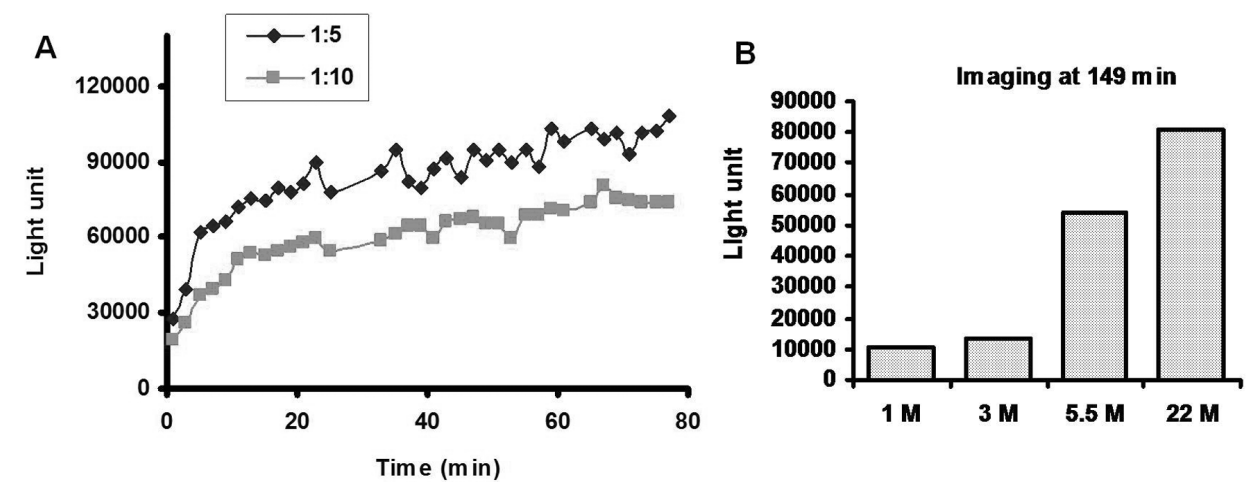

Figure 1. Agarose gels provide a hypoxic environment for cells. (A) The dynamic light intensity was analyzed from $1 \%$ cylinder agarose gels with a diameter of $4.8 \mathrm{~mm}$ containing 1 million HL cells after 1 day cultured at normoxia. During imaging, gels were put in luciferin dilutions. It is noted that the light intensity was affected by the presence of luciferin (1:5 vs 1:10). (B) Quantification of light intensity from gels with different cell densities at the end of imaging of 149 minutes. $1 \%$ cylinder-like agarose gels with a diameter of $4.8 \mathrm{~mm}$ contained 1, 3, 5.5 and 22 million HL cells respectively. Total light intensity was quantified by Image $\mathrm{J}$. 

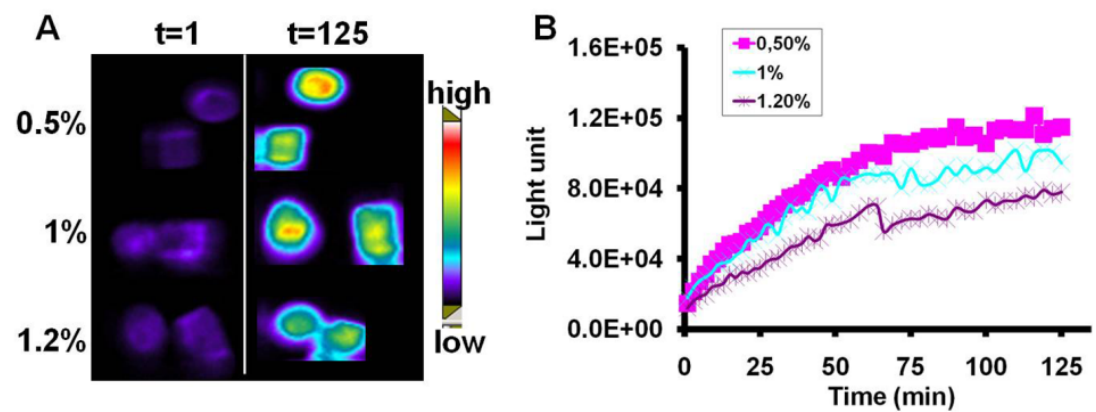

Figure 2. Effect of agarose concentration on oxygen diffusion. (A) Bioluminescent images of agarose gels at different concentrations containing 1 million HL cells each. The images shown here were from the first time point $(\mathrm{t}=1 \mathrm{~min})$ and the end time point $(\mathrm{t}=125 \mathrm{~min})$. Pseudocolor represents light intensity. (B) Dynamic light intensity from gels shows a reverse relationship between gel concentration and HRE-Luc activity.

To demonstrate that oxygen is rate limiting in the gel, we seeded 1 million HL cells either in a cylinder hydrogel of $4.8 \mathrm{~mm}$ or in a well of 24-well plate for one day. Cells were lysed and luciferase activity was quantified using a biochemical luciferase assay. The cells from the gels had a $1.7 x$ higher luciferase activity than HL cells in 2D culture (Fig. 3A). Meanwhile we performed BLI on those samples (Fig. 3B). In 2D culturing, the luminescent signal was immediately at its highest level, whereas in hydrogels, we again observed the gradual increase, which demonstrate that the gradual increase in light emission is due to the slow rate of luciferin diffusion rather than for instance the kinetics of the luciferase enzyme itself. These data show that the HRE-Luc reporter activity depends both on hypoxia and the luciferin diffusion properties of the material used. Hence, HRE-Luc should only be applied to materials with equal diffusion properties.
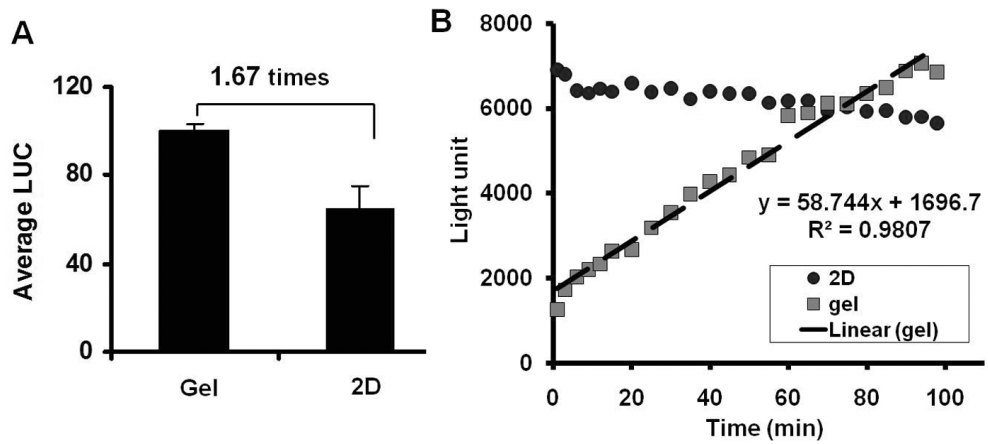

Figure 3. Limited luciferin diffusion in hydrogels. (A) Average cellular luciferase activity in a 3D hydrogel compared to 2D culture. Values are mean \pm SD (standard deviation) $(n=2)$. (B) Quantification of dynamic bioluminescence intensities of 3D hydrogel constructs versus $2 \mathrm{D}$ culturing over time. 


\section{Luciferin as a model molecule for diffusion in hydrogels}

A cell line (CL2) was created in which luciferase is driven by a constitutively active promoter (CMV). Given a certain number of viable CL2 cells in a gel, the slope of the light intensity plotted against time indicates the luciferin diffusion rate in agarose gels. We anticipated that the plateau of light intensity would not be affected by the gel percentage, given that sufficient time for luciferin is allowed to reach equilibrium. To test this, one million CL2 cells were seeded in either $0.5 \%$ or $1.2 \%$ agarose cylinder gels of $4.8 \mathrm{~mm}$ and BLI was performed after 1 day of culture. Cell viability staining revealed that cells were alive (data not shown). Fig. 4A clearly depicts the temporal changes in light intensity, with at early time points high light intensity at the periphery of the gel and lower light intensity in the center. As expected, the slope of the $1.2 \%$ gel was lower than that of the $0.5 \%$ gel (Fig. 4B), reflecting slower luciferin diffusion in the former. Based on the two slopes, we calculated that the luciferin diffusion rate in $0.5 \%$ agarose gel was $1.35 \mathrm{x}$ higher than in a $1.2 \%$ agarose gel. However, the plateau of the luminescent signal was not the same for the two constructs. The signal in $1.2 \%$ agarose gels never reached that of $0.5 \%$, indicating that both the slope and plateau level of the signal reflects the rate of luciferin diffusion.

A

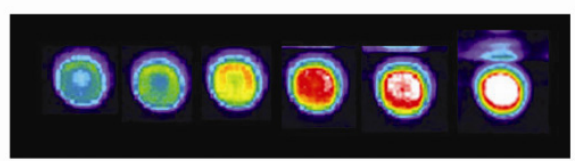

Time

B
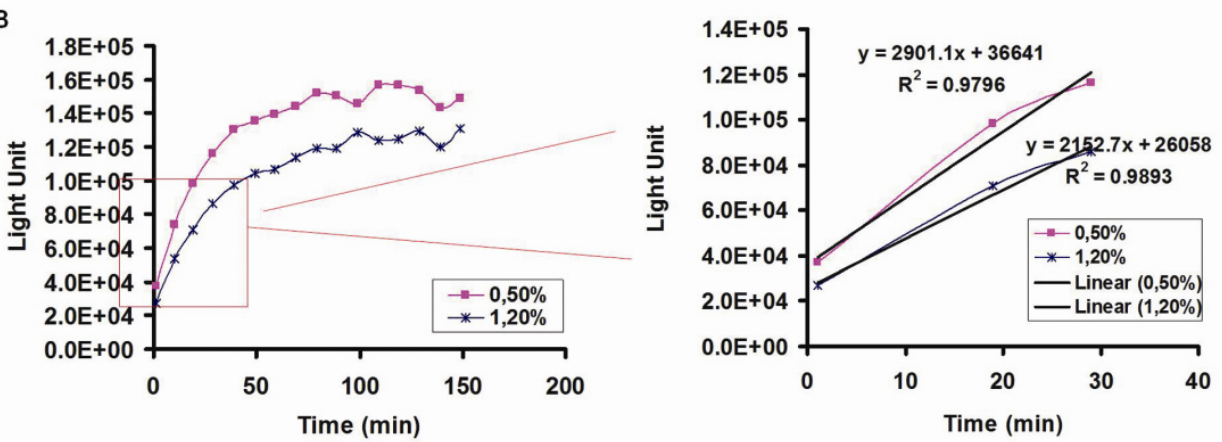

Figure 4. Luciferin diffusion in agarose gels is affected by gel concentration. (A) Pseudocolor images of agarose gels with CMV-Luc2 $\mathrm{CHO}$ cells showed that light intensity increased with luciferin diffusion longitudinally. (B) Dynamic analysis of CMV-Luc activity from agarose gels showed the the gel concentration dependent diffusion. $0.5 \%$ gel has a higher diffusion rate, indicated by the slopes of the fitted curves. 


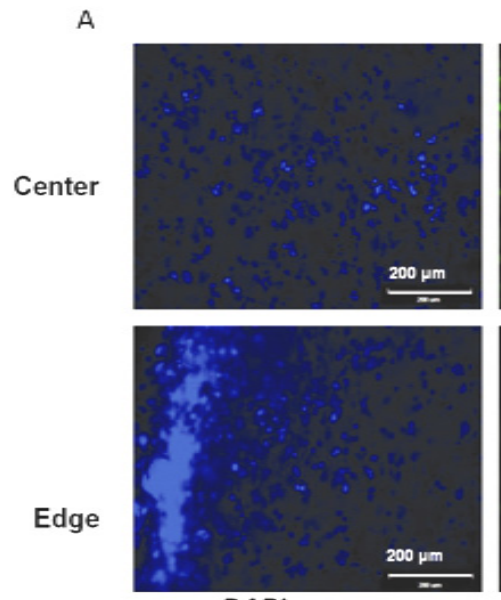

DAPI
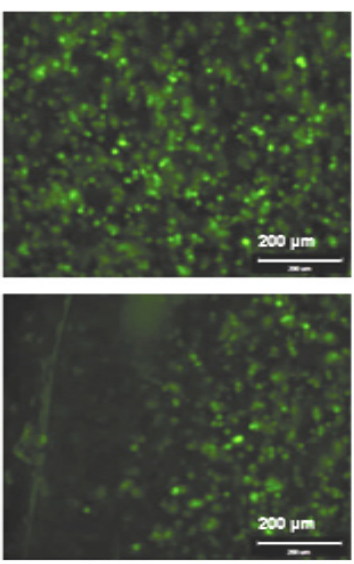

GFP
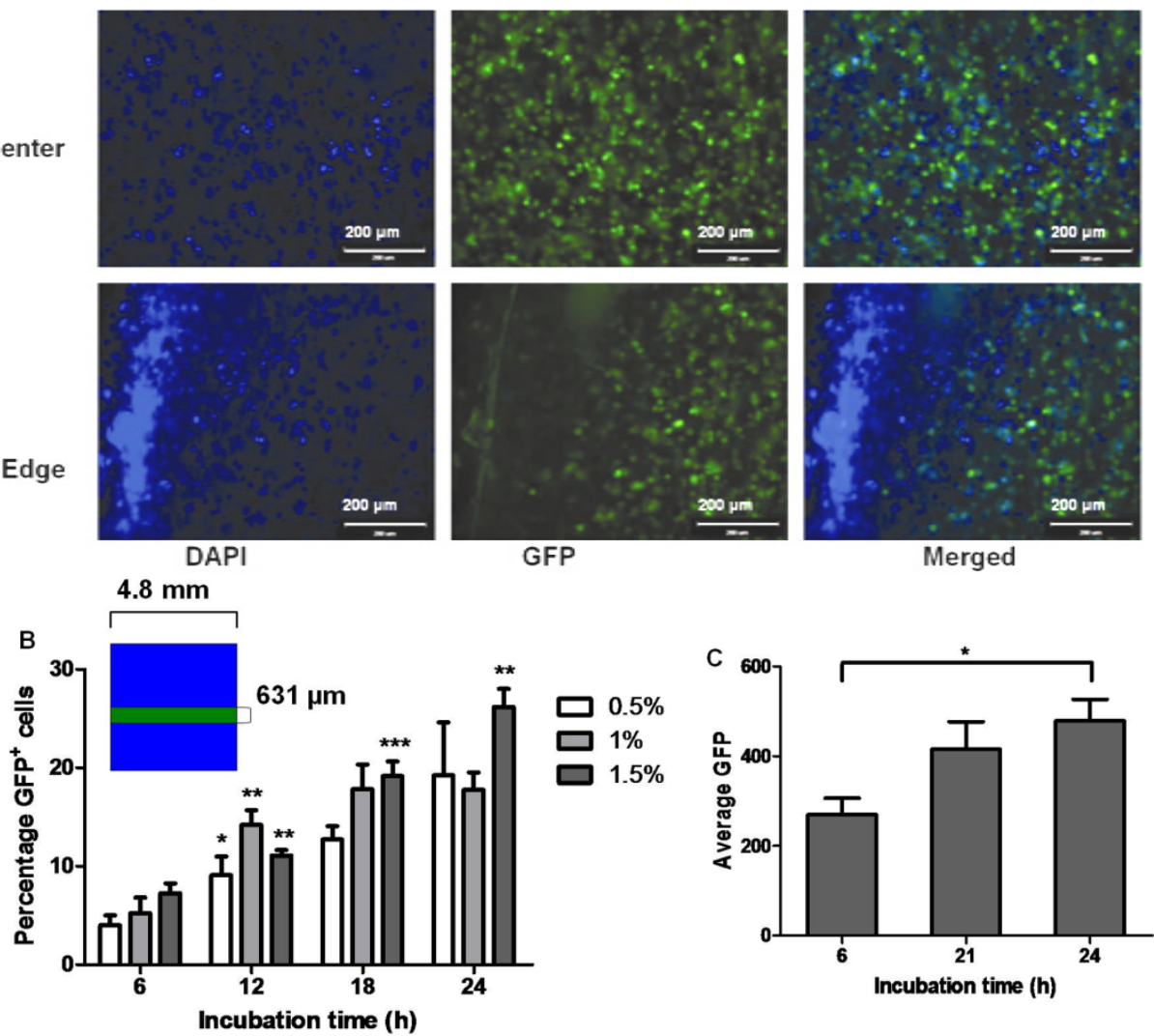

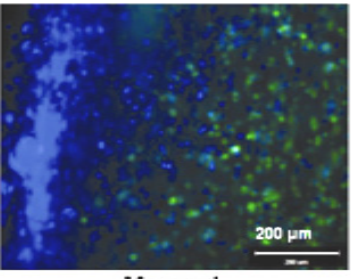

Merged

D

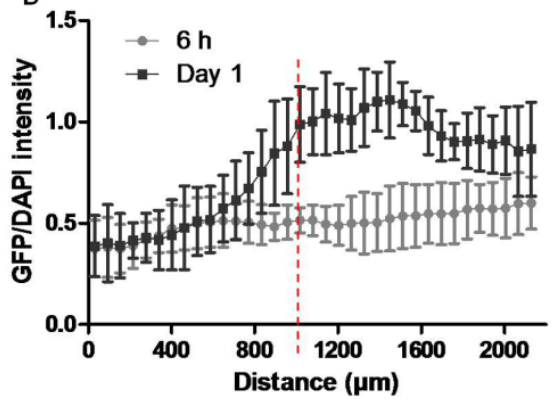

$4.8 \mathrm{~mm}$

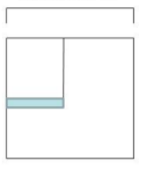

Figure 5. Hypoxia in hydrogels monitored by HRE-GFP CHO cells. (A) Heterogeneous GFP expression from $\mathrm{HG}$ cells after one day incubation at normoxia. 1 million $\mathrm{HG}$ cells were seeded in an $\varnothing 4.8 \mathrm{~mm}$ cylinder $1 \%$ agarose gel. DAPI stained the cell nucleus. (B) The number of GFP positive cells increased with incubation time. GFP expressing cells were counted in an area of $4.8 \mathrm{~mm} \times 631 \mu \mathrm{m}$ as indicated by confocal fluorescent microscopy. Average number of DAPI cells counted per gel slice is around $1.44 \times 10^{4}$. Significant comparison at different time point in the same gel density was analyzed by two-way ANOVA, $* * p<0.01, * * *$ $p<0.0001$. (C) The average GFP intensity throughout the whole gel slide increased in time. The statistical analysis was analyzed by one-way ANOVA, $* p<0.05$. (D) The average GFP intensity depended on the diffusion distance within the gel. The average GFP intensity was calculated by normalizing the GFP expression to the DAPI expression. Values represented mean \pm SD $(n=3-5)$. 


\section{HRE-GFP revealed progressive hypoxia in $3 D T E$ gels}

To exclude the influence of luciferin diffusion on the hypoxic signal and to be able to image a gradient of hypoxia within gels at the single cell level, we created the HRE-GFP CHO (HG) cell line. Using confocal imaging, single cell hypoxia measurements can be performed. 1 million HG cells were seeded in $1 \%$ agarose cylinder gels with a diameter of $4.8 \mathrm{~mm}$ and cultured for one day. Fluorescent microscopy showed that GFP positive cells were not homogenously distributed throughout the gel (Fig. 5A). In the center, nearly all cells clearly expressed GFP, whereas in the periphery hardly any GFP positive cells could be detected. We then temporally quantified the number of GFP positive cells within the whole gel. At early time points up to 4 hours of culture, no GFP positive cells were observed. After 6 hours, GFP expression could be observed and increased with time (Fig. 5B). Correspondingly the average GFP expression from the whole cross sections of gels increased with longer incubation time (Fig. 5C). No significant differences in GFP expression were found between different gel densities. Moreover the GFP expression along the depth to the central gel was evaluated and a critical distance around $1000 \mu \mathrm{m}$ was noticed (Fig. 5D), which corresponded well to what previously reported by using an $\mathrm{O}_{2}$ microelectrode sensor in tissue engineered cartilaginous constructs [150].

\section{Size-dependent molecular diffusion in 3D TE gels}

Reporter assays are an indirect method to assess diffusion of molecules, oxygen and luciferin. To directly monitor molecular diffusion in tissue engineering constructs, we used fluorescence recovery after photobleaching (FRAP). To this end, fluorescent dextran molecules of different molecular weights were mixed in agarose gels and FRAP was determined. Table 1 shows that the molecular weight of the dextrans is in the same range as that of biologically relevant molecules. Fig. 6A depicts the different stages in a FRAP experiment with $70 \mathrm{kD}$ dextran in a $0.5 \%$ agarose gel. In dried samples, little or no recovery was observed, because the molecules were immobilized due to lack of water. In normal gel samples, bleaching and recovery of fluorescent signal could be clearly monitored (Fig. 6B). It was noted that bleaching in the normal samples was not as efficient as in the dried samples and that the bleaching efficiency for the bigger dyes was higher than for the smaller dyes. This was probably due to that the particles partly diffuse back into the bleached area during the bleaching process. This leads to inefficient bleaching, especially for the smaller dyes. The intensity of the 389Da and $3 \mathrm{kDa}$ dye were only decreased 
with respectively $5 \%$ and $8 \%$ of the initial fluorescence, while bleaching the $10 \mathrm{kDa}$ and $70 \mathrm{kDa}$ dyes resulted in a $30 \%$ fluorescence drop.
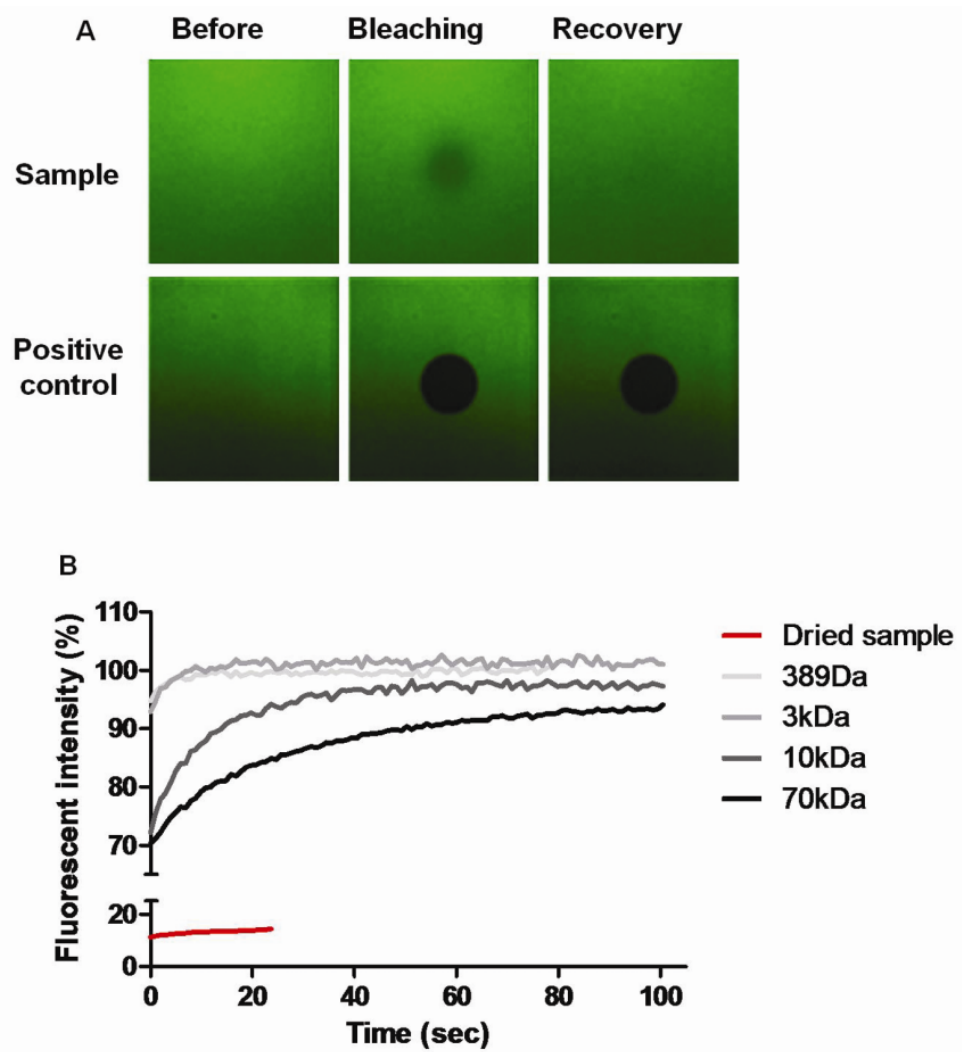

Figure 6. FRAP imaging of particles' diffusion in hydrogels. (A) Different stages of FRAP of DextranFITC dye (from left to right) in normal and dried situation (control). $0.5 \%$ agarose gel mixed with $5 \mu \mathrm{M}$ Dextron-FITC dye (MW 70,000Da). (B) Recovery curves of DexF dyes and dried control. The quantitative recovery of the fluorescent signal is visualized by plotting the average intensity of the bleached area $\left(f_{R O I}\right)$ normalized to the background intensity $\left(\mathrm{f}_{\text {ref }}\right)$ versus time.

Following we performed the FRAP of $70 \mathrm{kD}$ dextran at different gel concentrations. Fig. 7A shows the longitudinal $70 \mathrm{kD}$ dye intensity during the whole FRAP process in $0.5 \%, 1 \%$ and $2 \%$ agarose gels. The calculated half recovery time $T_{1 / 2}$ increased with gel concentrations, indicating a lower diffusion rate in high concentration gels (Fig. 7B). Different dye concentrations did not affect the $T_{1 / 2}$ value, demonstrating that the diffusion rate is a property of the material, and independent of the dye concentration. We calculated the diffusion coefficient (Table 2 ). It is noted that the $T_{1 / 2}$ slightly but significant increased with doubling gel concentration with a factor of 0.14 . 

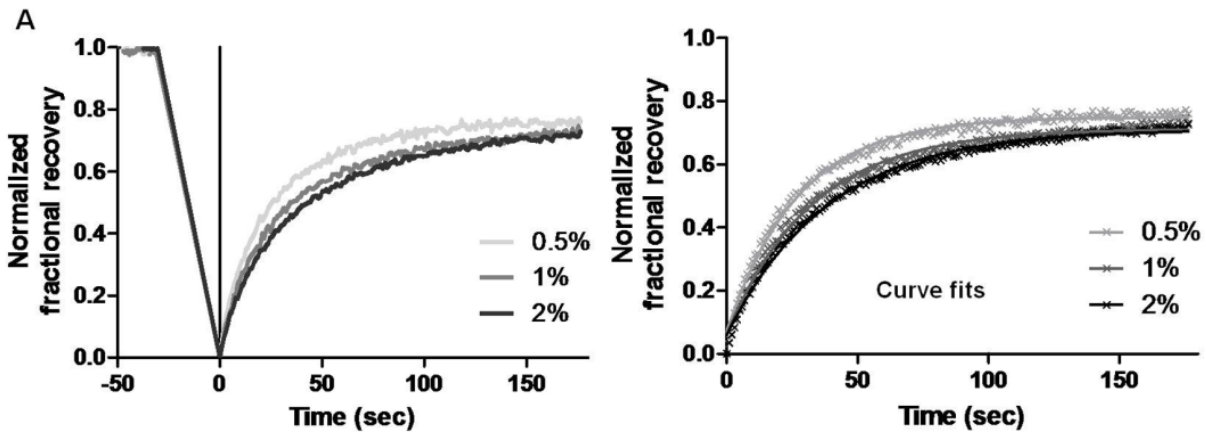

B

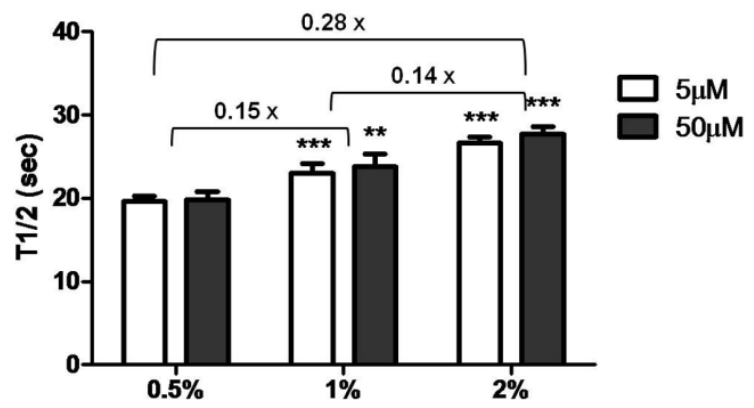

Gel concentration

Figure 7. The influence of gel concentration and dye concentration on $T_{1 / 2}$. (A) FRAP curves and curve fits of 70,000Da DexF in different agarose gel concentrations. (B) Half recovery times of DexF dye (MW $70,000 \mathrm{Da})$ at two different concentrations in agarose gels with varied concentrations. Values represented mean $\pm \mathrm{SD}(\mathrm{n}=3-5)$. The gel concentration had a significant effect on $\mathrm{T}_{1 / 2}(* * p<0.01, * * * p<0.001)$, while the dye concentration didn't, which was analyzed by two-way ANOVA. Asterisks $\left(^{*}\right)$ indicate significant changes in $T_{1 / 2}$ values compared to the values of the same dye concentration in the previous gel density.

Table 2. The effect of the dye and gel concentration on $D$.

\begin{tabular}{|c|c|c|}
\hline \multicolumn{3}{|c|}{ Diffusion coefficients $\left(\mu \mathrm{m}^{2} \mathrm{~s}^{-1}\right)$} \\
\hline \multirow{2}{*}{ Agarose gel (\%) } & \multicolumn{2}{|c|}{ Dye concentration } \\
\cline { 2 - 3 } & $5 \mu \mathrm{M}$ & $50 \mu \mathrm{M}$ \\
\hline 0.5 & $6.2 \pm 0.2$ & $6.1 \pm 0.3$ \\
\hline 1 & $5.3 \pm 0.2$ & $5.1 \pm 0.3$ \\
\hline 2 & $4.5 \pm 0.1$ & $4.4 \pm 0.1$ \\
\hline
\end{tabular}

Next, we investigated the effect of molecular sizes on the $T_{1 / 2}$. The dynamic curves in Fig. 8A and the corresponding $\mathrm{T}_{1 / 2}$ values (Fig. 8B) demonstrate that bigger particles have higher $T_{1 / 2}$ values, indicating lower mobility. Increasing the dye size from $10 \mathrm{kDa}$ to $70 \mathrm{kDa}$ for example, led to a more than 2.7 times slower recovery, which was in agreement of previous 66 
reports [180, 181]. Again, the effect of the density of the gel on the mobility of the particles was observed. Table 3 summarizes the diffusion coefficients based on the $\mathrm{T}_{1 / 2}$ values.
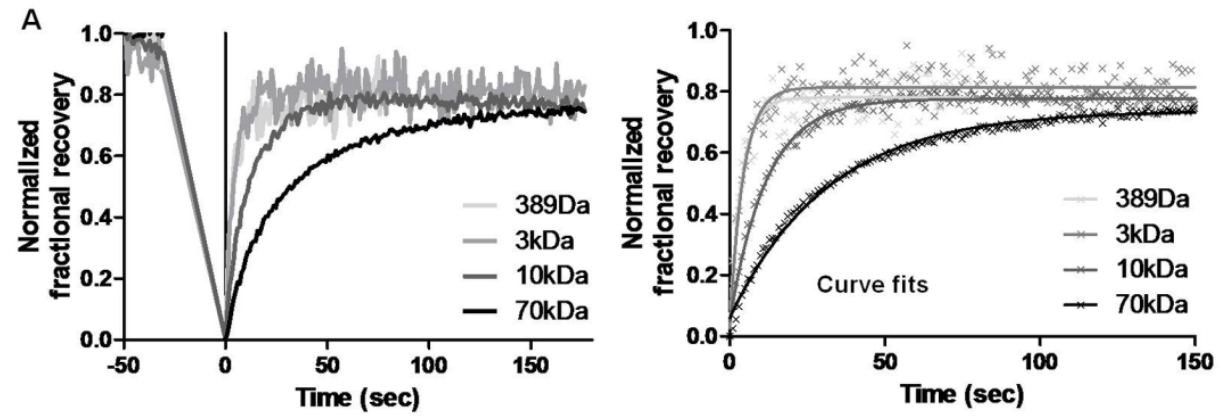

B
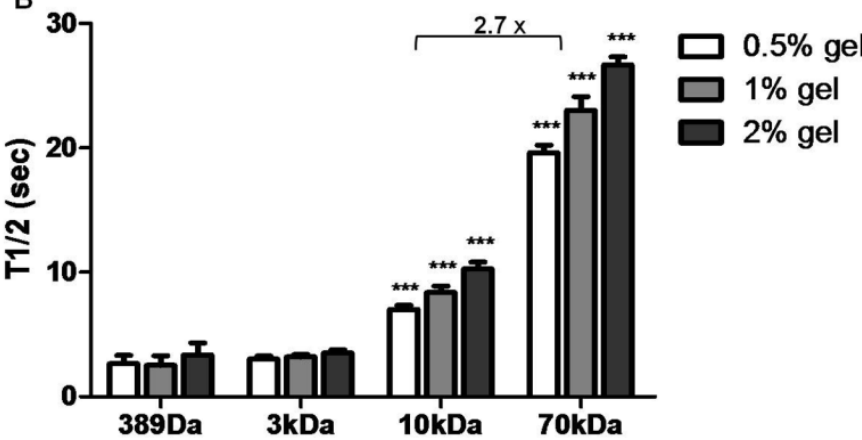

Dye size

Figure 8. The diffusion rates of dextrans in agarose gels. (A) FRAP curves and curve fits of different sized molecules in $1 \%$ agarose gels. (B) Half recovery times of DexF particles in different agarose gel concentrations. Values represented mean $\pm \mathrm{SD}(\mathrm{n}=3-5)$. Statistical study was performed by two-way ANOVA, $* * * p<0.0001$. Asterisks $(*)$ indicate significant changes in $\mathrm{T}_{1 / 2}$ values compared to the $\mathrm{T}_{1 / 2}$ values of a smaller dye in the same gel density.

Table 3. The effect of the dye size on $D$.

\begin{tabular}{|c|c|c|c|c|}
\hline \multicolumn{5}{|c|}{ Diffusion coefficients $\left(\mu \mathrm{m}^{2} \mathrm{~s}^{-1}\right)$} \\
\hline \multirow{3}{*}{ Agarose gel (\%) } & \multicolumn{4}{|c|}{ Dye size $(\mathrm{kDa})$} \\
\cline { 2 - 5 } & 0.389 & 3 & 10 \\
\hline 0.5 & $48.2 \pm 13.6$ & $40.5 \pm 3.5$ & $17.3 \pm 0.8$ & $6.2 \pm 0.2$ \\
\hline 1 & $51.5 \pm 15.5$ & $38.1 \pm 2.5$ & $14.5 \pm 0.9$ & $5.3 \pm 0.2$ \\
\hline 2 & $33.4 \pm 14.3$ & $34.5 \pm 2.3$ & $11.8 \pm 0.6$ & $4.5 \pm 0.1$ \\
\hline
\end{tabular}




\section{DISCUSSION}

Previously, we have investigated hypoxia in HRE-Luc cells in tissue engineered constructs in vitro using a biochemical assay in which cells are lysed prior to analysis (chapter 3). In this study, we have exploited the application of this reporter system in $3 \mathrm{D}$ gel constructs by bioluminescent imaging where luminescence is assayed in real time in living cells. In this situation, luciferin has to diffuse from the medium through the graft to reach the luciferase transgenic cells. We noted that luciferin distributes very rapidly throughout a luciferase transgenic mouse after intraperitoneal injection, and the light intensity reaches a plateau within 10 minutes (chapter 5). Evidently, the strength of the luminescent signal produced by the HRE-Luc construct depends on the level of hypoxia and the number of transgenic cells in the graft. But in this manuscript we observed a reverse relationship between agarose concentration and light intensity in a 3D agarose gel for both the HRE-Luc and the constitutively active CMV-luc transgene. It implies that luciferin diffusion is rate limiting in this system and determines the signal plateau in addition to cell number and degree of hypoxia. We conclude that the luciferin diffusion properties of the region of interest should be identical in all experimental groups when the HRE-Luc construct is used for BLI. In fact, this conclusion applies to BLI in general because luciferin diffusion is necessary in most BLI applications using firefly luciferase.

Nutrient diffusion in biomaterials depends on material chemistry, physical properties and its architecture. In addition, the diffusion properties can also be influenced by cells in the graft and by the extracellular matrix they produce [45]. As discussed above, luciferase transgenic reporter systems have a drawback in that they depend on the diffusion properties of the graft, but every drawback has its merit, so we used this property to visualize the process of luciferin diffusion non-invasively in 3D gels. CMV is a constitutively active promoter and CMV-Luc cells are often used to study cell proliferation both in vitro and in vivo because the light intensity from CMV-Luc cells relies only on cell number and luciferin diffusion, as demonstrated [82]. Hence, the slopes of the dynamic curves from gels incubated with CMV-Luc2 cells reflected the rate of luciferin diffusion in biomaterials.

To circumvent diffusion of luciferin as a variable in optical imaging of hypoxia, we replaced luciferase by green fluorescent protein (GFP), which allowed us to measure hypoxia at the cellular level using confocal microscopy. 
Indovina and colleagues used spheroids of HRE-GFP transgenic MG63 cells to reveal oxygen depletion in the core of the cell aggregates [184]. Furthermore, Harada and colleagues generated Hela/HRNG cells (hypoxia-dependent red fluorescence and normoxia-dependent green fluorescence) in which the color changed in response to the oxygen tension to study tumor development [185]. In this manuscript, we have investigated oxygen availability of cells in a tissue engineering construct by evaluating GFP expression. We clearly observed a lack of oxygen in cells beyond $200 \mu \mathrm{m}$ from the periphery of the gel. We also monitored hypoxia dynamically and observed an increasing number of GFP positive cells as soon as 6 hours after gel formation. However, there was no significant effect of gel concentration on hypoxia. We conclude that the HREGFP reporter can be a very valuable tool to monitor oxygen availability in tissue engineered constructs. With HRE as the promoter driving reporter gene expression, the oxygen level is assessed but the promoter may be specifically tailored to detect the availability of other molecules of interest in tissue engineered grafts. For instance, the presence and biological activity of bone morphogenetic protein (BMP) can be evaluated by using a BMP-responsive element as promoter [84].

Reporter systems depend on the response of a cell to the presence of certain molecules: oxygen and luciferin in this manuscript. As such, reporter systems monitor diffusion indirectly. To study the mobility of molecules with diverse sizes directly, FRAP was employed. We used fluorescently labeled dextrans in a molecular weight range representing small molecules such as oxygen, intermediate size molecules such as glucose and lactose and larger sized proteins. Table 2 and Table 3 demonstrated that the diffusion rate in agarose gels depended on gel concentration and particle size but was independent of the concentration of the fluorescent dextrans. However, it is noted that the diffusion coefficient D of the smallest dye (389D) was similar to the $\mathrm{D}$ of $3 \mathrm{kDa}$ dye. The inefficient bleaching of the small dyes $(389 \mathrm{Da}$ and $3 \mathrm{kDa})$ as mentioned before results in less accurate curve fits ( $\mathrm{R}^{2}$ values: 0.79 for $3 \mathrm{kDa}$ dye compared to 0.99 for $70 \mathrm{kDa}$ dye), and thus less accurate $\mathrm{T}_{1 / 2}$ values and diffusion coefficients. In addition, the poor temporal resolution of the microscope in the FRAP setup might play a role in obtaining inaccurate diffusion coefficients. With the current settings it takes 0.985 seconds to scan one image, limiting the resolution in time which is especially critical for detection of small molecules that diffuse fast. 
Theoretical FRAP models assume instantaneous bleaching, neglecting diffusion processes during the bleaching time. Hence, the time for photobleaching should be very short $(<5 \%)$ compared to the recovery time. In experiments depending on longer photobleaching times, the so-called corona effect results in an underestimation of the diffusion coefficient. The corona effect occurs through the exchange of bleached and unbleached particles in and out of the ROI during the relative long bleaching time, leading to an enlarged ROI. Weiss et al., demonstrated that a 5-fold increase in the number of repetitive ROI scans results in a 3 times lower diffusion coefficient [186]. To avoid the corona effect, it is important that the availability of fluorescent molecules in the sample is relatively high. This can be achieved by either using a big sample volume with a high concentration of fluorescent molecules, or by selecting a ROI that is small compared to the total size of the sample. Although in these experiments, the size of the ROI is very small compared to the total sample area $\left(380 \mu \mathrm{m}^{2} / 25 \mathrm{~mm}^{2}\right)$, the corona effect might have occurred in these experiments due to the very high number of repetitive scans (150 iterations for 30.4 seconds). Therefore, we used a normalized fractional recovery method $\left(f_{k}(t)=\frac{f_{R O I}(t) / f_{r e f}(t)-f_{R O I}(0) / f_{r e f}(0)}{f_{R O I}(\infty) / f_{\text {ref }}(\infty)-f_{R O I}(0) / f_{\text {ref }}(0)}\right.$ instead of $\left.f_{k}(t)=\frac{f_{k}(t)-f_{k}(0)}{f_{k}(\infty)-f_{k}(0)}\right)$ to correct for the background bleaching as described in the method part. In this study, we have demonstrated that FRAP is a very useful tool to screen for molecules with a favorable diffusion characteristic, e.g. by changing their size or chemistry. Improving molecular diffusion is an important step towards modeling, and eventually improving, the transport of nutrients or certain growth factors in tissue engineering constructs. Agarose gels were mainly used in this manuscript, which is a simplified representation of human tissue. In order to model the diffusion in a more natural situation, FRAP studies need to be extended to more complex grafts, e.g. composite grafts with ECM proteins or tissue engineered grafts.

In this chapter, we have monitored the distribution and diffusion of nutrients in cell-biomaterial 3D construct. In conclusion, we have demonstrated that optical imaging with reporter genes is a versatile tool to study nutrient or growth factor availability in 3D tissue engineered constructs. FRAP could provide direct knowledge on nutrient diffusion in biomaterials. Thus, optical imaging in tissue engineering exceeds visualizing cells. Combined with appropriate cellular assays, it can also be very useful to evaluate biomaterial 
properties to optimize scaffold structural design and modification to improve the availability of nutrient or growth factors to cells.

\section{ACKNOWLEDGEMENTS}

We thank dr. Shinae Kizaka Kondoh from Kyoto University, Kyoto, Japan, and dr. Kiyoshi Nose from Showa University, Tokyo, Japan for providing the HRE-Luc plasmid DNA and A4-4 cells respectively. This research was sponsored by a grant from Senter/Novem. 



\section{ABSTRACT}

The luciferase transgenic mouse model is a very promising tool for noninvasive, quantitative and longitudinal evaluation of the expression of an interested gene. The aim of this study was to evaluate two transgenic mouse models in which the luciferase gene is driven by bone specific regulatory elements from the mouse collagen $\alpha(1) \mathrm{I}$ (Col(I)-Luc) and the human osteocalcin gene (hOC-Luc) respectively, during bone development and remodelling by bioluminescent imaging (BLI). We observed strong luciferase activity in skeletal tissues of Col(I)-Luc mice, but the light intensity declined with postnatal bone development. Furthermore, luciferase activity was enhanced in two models for tail bone repair. Moreover, we could monitor the process of ectopic bone formation induced by recombinant human bone morphogenetic protein 2 (rhBMP2) using BLI. In contrast, we observed strong inter-animal heterogeneity of luciferase in the level and location of signal in hOC-Luc transgenic mice. Upon subcutaneous implantation of biomaterials, light was detected from nearly all the wound sites including mock controls without materials during the first few weeks. Moreover, when ceramic scaffolds loaded with rhBMP2 were implanted subcutaneously, no light was detected from the graft up to 4 months post surgery, even though ectopic bone was formed. We conclude that Col(I)-Luc transgenic mice can be applied in the field of bone tissue engineering for monitoring the bone repair processes and to assess osteoinductive molecules or scaffolds.

\section{INTRODUCTION}

The most used clinical therapies for targeting osteoporosis, bone fracture and bone loss are the administration of bone anabolic molecules, such as parathyroid hormone (PTH) and bone morphogenetic protein (BMP) or to implant bone-inducing grafts. To assess the therapeutic efficacy, animal models are a logical choice to approach the clinical physiology and anatomy as close as possible. The most robust technique for the evaluation of de novo bone formation is post-mortem histological analysis. Because histology is an endpoint evaluation and large inter-animal variation is observed, typically large numbers of animals are required to assess statistically significant temporal results. In search of alternatives, much progress has been made on new 
approaches for in vivo imaging in small animal models such as ultrasound imaging, single photon computed tomography (SPECT), positron emission tomography (PET), magnetic resonance imaging (MRI) and X-ray computed tomography (CT) [61, 62]. These imaging modalities offer scientists the spatial and temporal information in a faster and more convenient manner. $\mu \mathrm{CT}$ is an efficient and reliable way to examine the anatomy of hard tissues, however its radiation environment and long scanning time limit its application as a routine method on living animals. MRI has been also applied to determine bone formation, but it lacks genetic information. Although SPECT and PET can offer molecular imaging by tracking radioactive isotopes, they are limited to routine research on gene and protein expression due to technical hurdles.

In the last decade, optical imaging using a reporter gene (either fluorescence or luminescence) is broadly used to study gene function [62, 69]. Bioluminescent imaging (BLI) with luciferase is widely applied to monitor gene expressions and cellular activities both in vitro and in vivo, because the regulatory elements driving the luciferase gene can be tailored specifically for particular applications [76-78]. BLI is based on the luciferase-luciferin reaction in which photons are emitted which can be captured using cooled chargecoupled device (CCCD) camera. BLI does not suffer from internal background noise due to employing an exogenous gene. Besides the substrate luciferin is nontoxic. As such, it serves as a non-invasive, quantitative and repetitive imaging tool on transgenic expression in individual organisms.

In bone tissue engineering (BTE), cell proliferation and differentiation can be monitored depending on the regulatory sequences driving lucfierase expression. For instance, the constitutively active cytomegalovirus (CMV) promoter can be used to monitor cell survival and proliferation [81, 82]. In addition, osteoblast-specific promoters have been evaluated as a tool for monitoring osteogenic differentiation including sequences derived from the Runx2/Cbfa-1 (RUNX), osteopontin (OPN), osteocalcin (OCN), collagen type 1a (COL), alkaline phosphatase (ALP) and bone morphogenetic genes [83, 84]. In addition, we investigated the cellular hypoxia/nutrient status during BTE using hypoxia responsive element (HRE)-luciferase transgenic cells (Chapter 3).

Mice transgenic for luciferase under the control of bone-specific reporter elements can potentially be used to investigate bone metabolism or ectopic bone formation. Previously, hOC-Luc transgenic mice were reported to have luciferase acitivity limited to bone tissue with enhanced activity during bone fracture repair and BMP2-induced bone formation [86, 87]. In addition, 
luciferase activity was enhanced upon injection of vitamin D3 [77]. Transgenic mice harbouring a rat col $1 \mathrm{a} 12.3 \mathrm{~kb}$ and $3.6 \mathrm{~kb}$ promoter segment driving GFP were created [72] and the former showed strong GFP activity in osteoblasts of bone, odontoblasts in teeth, while the latter had GFP positive cells in both osseous and non-osseous tissues including osteoclasts [73-75]. Similarly, a $2.3 \mathrm{~kb}$ pro- $\alpha 1$ (I) collagen promoter segment drove LacZ and luciferase expression in osteoblasts and odontoblasts, whereas a $3.2 \mathrm{~kb}$ fragment of this proximal promoter had an additional expression in tendon and fascia fibroblasts [187]. A minimal sequence of $117 \mathrm{bp}$ within the $2.3 \mathrm{~kb}$ promoter segment was indentified to specifically confer expression to osteoblasts [188]. Luciferase assays from the latter mice demonstrated that PTH treatment enhanced luciferase activity with increased bone formation from femur lysis but not from skin [96].

Evidently, the specificity of the regulatory segments strongly determines a potential application to study bone development. In this chapter, we evaluated the Col(I)-Luc and hOC-Luc mice for potential use in ectopic bone formation and bone remodeling using BLI.

\section{Part 1: Col(I)-Luc mice}

\section{MATERIALS AND METHODS}

\section{Animals}

$\mathrm{B}_{6} \mathrm{X} \mathrm{D}_{2} \quad \mathrm{~F} 2$ mice, with the luciferase gene under the control of a $4 \mathrm{x}$ multimerized 356-base pair segment of the mouse $\alpha 1$ (I) collagen promoter and a 220-base pair segment of the $\alpha 1(\mathrm{I})$ collagen minimal promoter (Col(I)luciferase), were kindly provided by dr. de Crombrugghe [188]. All experiments were approved by the local Ethical Committee for Animal Experimentation and in compliance with the Institutional Guidelines on the use of laboratory animals. Thermal cycle PCR (Eppendorf) on the luciferase gene was used for genotyping the transgenic mice. DNA was extracted from mice tail or ear by using QIAamp DNA mini kit (Qiagen) according to the manufacturer's protocols. Primers used: forward primer 5'GGGCGCGGTCGGTAAAGTTGT-3' and reverse primer 5'CACCTGCGTCGAAGATGTTGG-3'. 


\section{In vivo imaging in time scale}

To study the activity of this promoter longitudinally, Five 5-week-old male mice were imaged for 6 months. Animals were anaesthetized with ketamine, xylazine and atropine solution (ratio of 1:1.25:0.75, KXA) by an intramuscular injection at $0.2 \mu \mathrm{l} / \mathrm{g}$ body weight. Next, mice were shaved and injected intraperitoneally with D-luciferin dissolved in PBS $(125 \mathrm{mg} / \mathrm{kg}$ body weight, Synchem Chemie, Kassel, Germany). BLI was performed for 30 minutes with a Roper Bioluminescence Imaging System, Princeton Instruments (Trenton, USA) at GDL, Utrecht, the Netherlands. The CCCD camera was a LN/CCD-1300EB with a 50-mm F1.2 Nikon lens (Roper Scientific). Image analysis was performed using Metamorph software (Universal Imaging Corp., West Chester, USA). The quantitative data were generated by averaging light intensities from 10-minute imaging after the light intensity reached a plateau after 20 minutes luciferin injection.

\section{Cell culture}

Mouse mesenchymal stem cells (MSCs) were isolated according to the protocol described previously [189]. Briefly, femurs were extracted from 8week-old mice, and bone marrow was flushed into a culture flask using CIM medium, consisting of RPMI 1640 (Invitrogen) supplemented with 9\% fetal bovine serum (Cambrex), 9\% horse serum (Hyclone), 100U/ml penicillin (Invitrogen), $100 \mu \mathrm{g} / \mathrm{ml}$ streptomycin (Invitrogen) and $12 \mu \mathrm{M}$ L-glutamine (Invitrogen). Medium was re-plated into a new flask after 24 hours and then refreshed every 3 days. After 3 passages, cells were changed into CEM medium (Iscove modified Dulbecco medium (IMDM) supplemented with $9 \%$ fetal bovine serum, $9 \%$ horse serum, $100 \mathrm{U} / \mathrm{ml}$ penicillin, $100 \mu \mathrm{g} / \mathrm{ml}$ streptomycin and $12 \mu \mathrm{M}$ L-glutamine). For osteogenesis, cells were seeded triplicate at $2,000 / \mathrm{cm}^{2}$ cells at passage 4 in both mineralization medium and control medium for 11 days. Mineralized medium was composed of CEM supplemented with 20mM $\beta$ glycerol phosphate (BGP, Sigma, St Louis, MO), 1nM dexamethasone (Sigma), $0.5 \mu \mathrm{M}$ ascorbate 2-phosphate (Sigma) and 100ng/ml human recombinant bone morphological protein 2 (rhBMP2, Biodoor Biotechnology co., LTD, China). Control medium was CEM plus BGP. Total calcium deposition was assayed using a calcium assay kit (Sigma) according to the manufacturer's protocol. To visualize the mineralized area, one flask from control or mineralized group was stained with von Kossa staining as described previously [28]. For adipogenesis, $2,000 / \mathrm{cm}^{2}$ cells at passage 6 were cultured in both adipogenic medium (CEM 
supplemented with $5 \mu \mathrm{g} / \mathrm{mL}$ insulin (Sigma), $50 \mu \mathrm{M}$ indomethacin (Sigma), $1 \times 10^{-6} \mathrm{M}$ dexamethasone and $0.5 \mu \mathrm{M}$ 3-isobutyl-1-methylxanthine (IBMX; Sigma)) and control medium (CEM) for 13 days. The cells were then fixed with $10 \%$ formalin for 20 minutes at room temperature (RT) and stained with $0.5 \%$ Oil Red O (Sigma) in methanol (Sigma) for 20 minutes at RT.

\section{Imaging the bone repair model}

Five 10-week-old male Col(1)-Luc transgenic mice were used as one group for control, and in aother 5 male mice a $\varnothing 1 \mathrm{~mm}$ hole was created in the third vertebra from the base of the tail using a Dremel tool equipped with a 1/32 inch drill bit.p. During the operation, mice were anesthetized using Isofluorane. Bioluminescent imaging (BLI) was performed using a CCCD camera from Ivis (Xenogen), at the animal facility of Schering-Plough Research Institute, Oss, The Netherlands, every week for 8 weeks as mentioned above.

\section{Imaging the ovariectomy (ovx) model}

Ten 3-month-old female mice underwent bilateral ovx via the dorsal approach as previously described [190], and 10 sham mice where used as control. BLI was performed using a CCCD camera from Ivis (Xenogen) every week for 4 weeks. After 4 weeks, $40 \mu \mathrm{g} / \mathrm{kg}$ (body weight) rhPTH was i.p. injected to 5 sham and 5 ovx mice every day for 5 days per week for 6 weeks, and the other mice were injected with saline as controls. $\mu \mathrm{CT}$ was performed to validate ovx and PTH effects post-mortem. During the operation and imaging, mice were under anaesthesia by Isofluorane.

\section{Micro Computer Tomography $(\mu C T)$}

For $\mu \mathrm{CT}$ scanning, femurs from both control and treated groups were isolated post-mortem and positioned as described by Stock et al.[191], scanned and trabecular bone mineral density was determined. In the tail-drilling experiment, mouse tail vertebrae from all groups were imaged on day 0 and at the last time point, and bone mineral density was determined. A Skyscan 1076 MicroCT-40 system (Skyscan, Belgium) and associated software was used to collect the data and to reconstruct the slices of the volume. The X-ray tube was operated with photon energy of $70 \mathrm{kV}$, a current of $140 \mu \mathrm{A}$ and a $0.50 \mathrm{~mm}$ thick Al filter with a scanning width of $35 \mathrm{~mm}$ and pixel size $9 \mu \mathrm{m}$. Mean values $(\mathrm{n}=5)$ and standard deviations of Bone Volume $\left(\mathrm{mm}^{3}\right)$ for control and treated samples were compared using Graph Pad Prism. 


\section{Imaging ectopic bone formation and histological analysis}

Four 7, 8-week-old male mice were implanted subcutaneously with 3 BCP particles ( $\beta$-calcium phosphate ceramic particles of $2-3 \mathrm{~mm}$ in size) in one pocket, 3 particles with $5 \mu \mathrm{g}$ human recombinant bone morphogenetic protein 2 (rhBMP2, Hangzhou Biodoor Biotechnology co., LTD, China) in another pocket and one pocket was left empty. BLI using a Photon imager (Biospace lab, Paris) was performed every week for 10 weeks. Mice were euthanised using $\mathrm{CO}_{2}$ and samples were explanted, fixed in $4 \%$ paraformadehyde, dehydrated and embedded in methyl methacrylate (Sigma) for sectioning. Approximately $10 \mu \mathrm{m}$ thick, undecalcified sections were processed on a histological diamond saw (Leica saw microtome cutting system). The sections were stained with basic fuchsin and methylene blue to visualize bone formation. The sections were scanned using a Minolta Dimage Scan. Bone formation is expressed as the percentage of bone areas over the total available pore area using Adobe Photoshop. Luminescent images were quantified using M3vision software (Biospace lab). During operation and imaging, mice were under anaesthesia by Isofluorane.

\section{RESULTS}

\section{Luciferase expression correlates with skeletal organs in Col(I)-Luc mice}

Collagen type I is the main protein component of bone. In this study, we used BLI as a tool to evaluate a Col(I)-Luc transgenic mouse line which harbours luciferase gene driven by a collagen I promoter gene fragment. BLI revealed that Col(I)-Luc mice expressed luciferase specifically from skeletal organs as showed in Fig. 1A. As it was found out early that this promoter sequence encoded osteoblasts in prenatal embryos, light was observed from the mandible, teeth, paws, lumbar vertebrae, caudal vertebrae, pelvis, ribs and femur but no light was from non-osseous tissues. We noted that the level of light expression displayed little inter-animal variation. In addition, mouse hair absorbed the light from the back completely (Fig. 1B), so in the following implantation experiments, the mice were all shaved. This mouse strain was heterozygous with majority brown pups and minor white ones. The brown mice also emitted very strong light from the paws, jaw and tail, but never from the body (Fig. 1C) indicating that the pigment absorbs light. After injection of Dluciferin, it took around 10 minutes for light intensity to reach the plateau level 
(Fig. 1D). Thus, all the quantified data in this study were taken 10 minutes later after substrate injection.
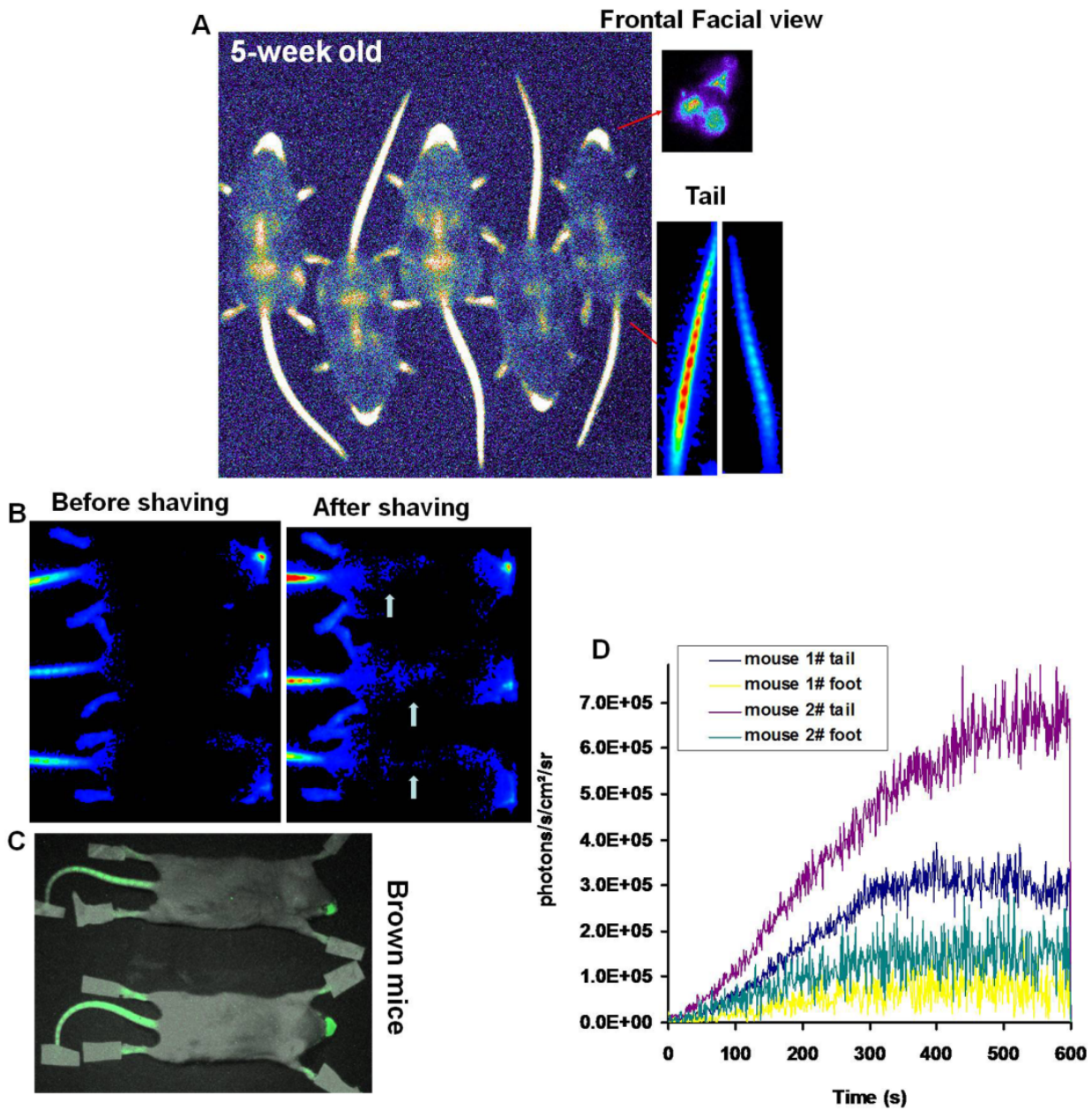

Figure 1. Luciferase activity was detected from bony parts of Col(I)-Luc mouse. (A) Luciferase is expressed from bony parts in the whole body with strongest expression at tail and jaw. This image was accumulated for 24-minute imaging after luciferin injection. The pseudocolor represented light intensity. (B) Images from before and after shaving the hair on the back. The acquisition time for this imaging was 2 minutes. (C) Skin pigment absorbed light. Brown transgenic mice expressed lucifease in a same manner as white ones except from the back even after hair shaved. (D) Kinetics of light emission following luciferin injection, expressed in terms of light unit. The quantification was done from tail and foot from two mice.

Next, the promoter activity was longitudinally evaluated. Clearly the luciferase expression from all skeletal organs declined dramatically within the first 7 months (Fig. $2 \mathrm{~A}$ and B), which reflects the postnatal development of skeleton. In particular, light emission from the tail decreased significantly within 11 weeks, while light from the jaw first declined but then remained 80 
stable for 18 weeks. We also evaluated luminescence in female mice but never found indications that the oestrous cycle impacts luciferase expression (data not shown).

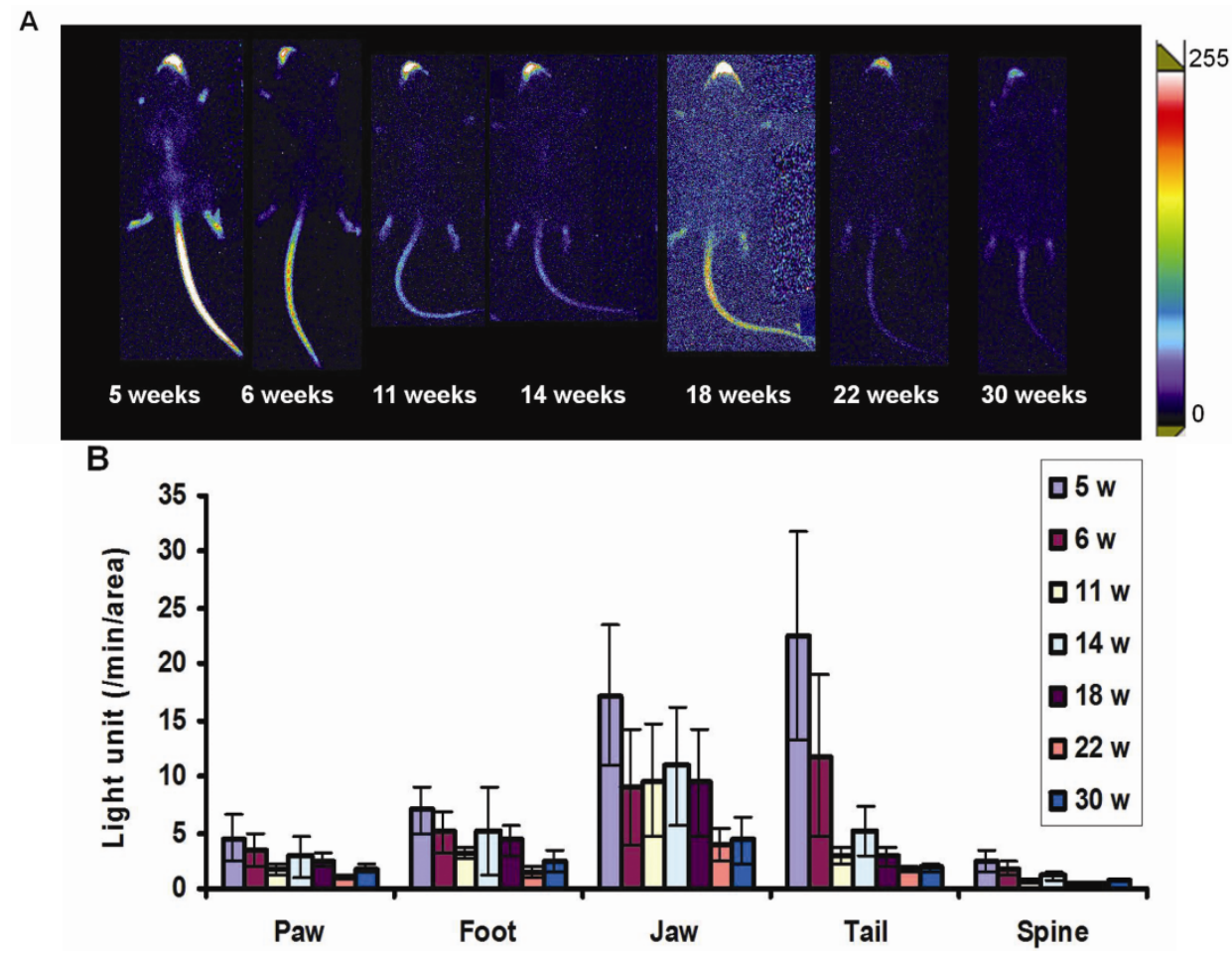

Figure 2. The regulation of bone specific collagen $I$ in postnatal skeleton development was evaluated by BLI. (A) Luciferase expression dropped longitudinally. The acquisition time for this imaging was 5 minutes. (B) The light unit here was normalized to the area of interested regions in one minute, and subtracted by the background noise. Values represent mean $\pm \mathrm{SD}(\mathrm{n}=5$, male).

\section{Absence of luciferase activity in osteogenic mesenchymal stem cells}

Mouse mesenchymal stem cells (mMSCs) isolated from the femoral bone marrow of Col(I)-Luc mice had a fibroblastic morphology in control medium and could differentiate along the adipogenic and osteogenic lineages (Fig. 3A and B). In adipogenic medium, Oil Red O-positive lipid droplets were observed in the vast majority of cells, in contrast to cells grown in control medium. Similarly, when cells were cultured in mineralization medium in vitro, von Kossa staining demonstrated mineral deposition by mMSCs grown only in mineralization medium. Surprisingly, we failed to detect luciferase activity in cells undergoing mineralization, even though the transgenic nature of the mMSCs was confirmed by PCR (Fig. 3C). We extracted mRNA from the control and mineralized cells to perform qPCR on the luciferase gene, but no 
luciferase expression could be detected (data not shown).
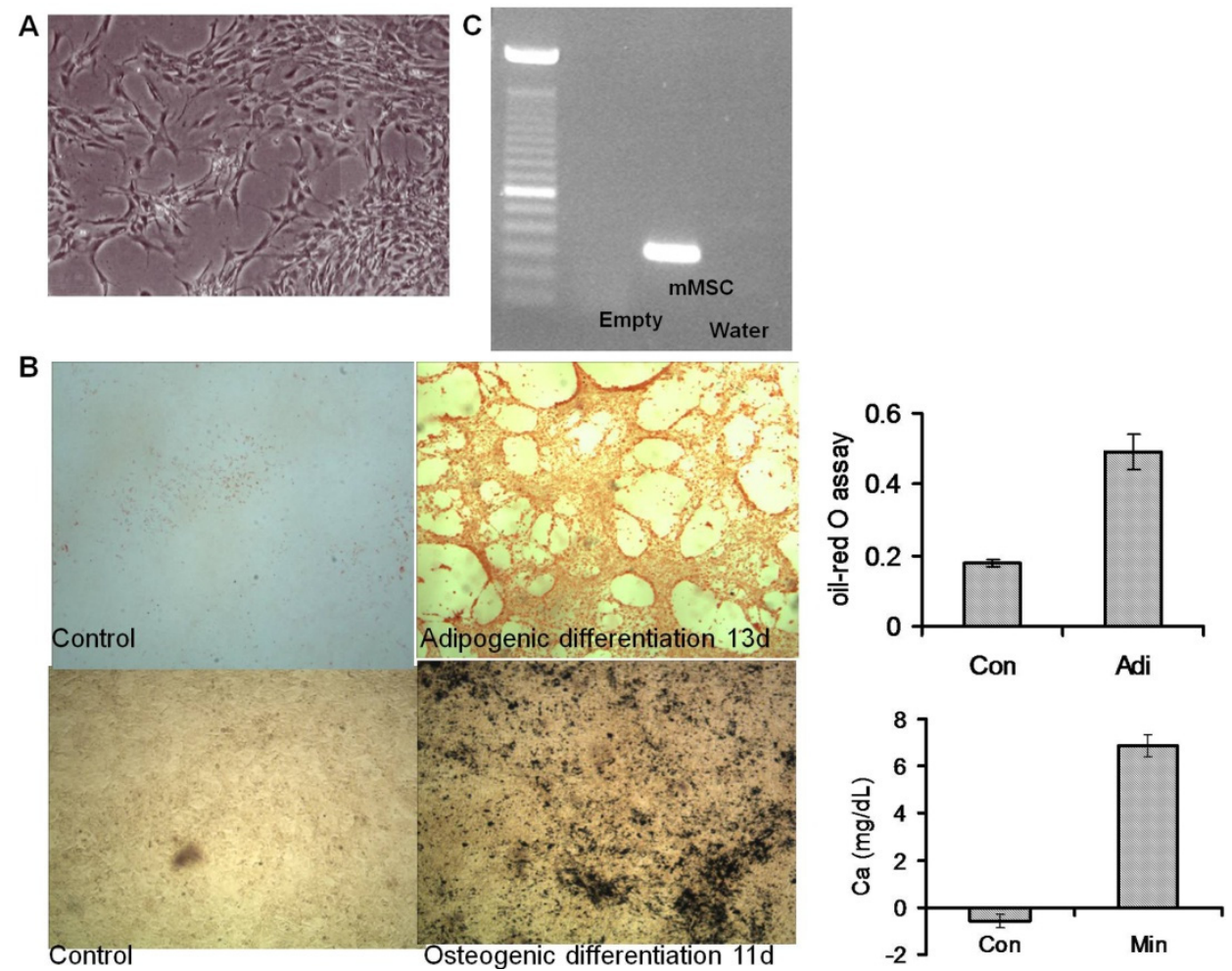

Figure 3. Mesenchymal stem cells from bone marrow didn't emit light during osteogenic differentiation. (A) mMSCs were isolated from femur bone marrow, and showed the typical fibroblastic morphology. (B) mMSCs are multipotent and can differentiate into adipogenic and osteogenic. The osteogenic differentiation was evaluated by Von Kossa staining for calcium deposition and Ca assay to quantify the minerals. Oil-red O staining of lipids and quantification demonstrated the adipogenic differentiation. Values represent mean \pm SD $(n=3)$. (C) A gel image after PCR demonstrated the mMSCs harbouring luciferase gene.

\section{Ovariectomy (OVX) model}

Estrogen is an important factor in bone homeostasis. Female menopausal osteoporosis is caused by reduced $17 \beta$-estradiol production in the ovaries. We evaluated the effect of ovariectomy and PTH injection on bone remodelling. Female Col(I)-Luc mice were either ovariectomised or shamed, and 4 weeks later, half of the mice from each group were treated with PTH for another 6 weeks. BLI was performed weekly and $\mu \mathrm{CT}$ of the femurs was performed at the end of the 10 week period. As expected, $\mu \mathrm{CT}$ revealed a reduction in trabecular bone mineral density (BMD) in OVX mice and PTH treatment restored BMD to control levels, thus validating the OVX and PTH effects (Fig. 4A). The light intensity shown in Fig. 4B was obtained from the 
tail, but represented light from other body parts (not shown). During the first 4 weeks post surgery prior to PTH administration, 5 OVX mice up-regulated luciferase expression significantly from day 13 till day 23, but in in another group of 5 ovx mice (OVX-PTH), there was a 2 -fold increase on day 13. No significant changes were observed in two sham groups (Fig. 4B). During the following 6-week PTH treatment, no profound effect of PTH on the BLI signal was observed.

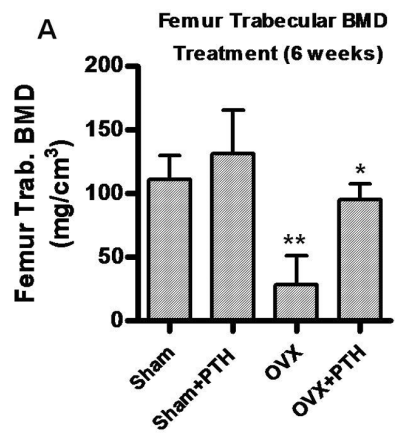

Figure 4. Bone remodelling by the ovariectomy (ovx). (A) $\mu \mathrm{CT}$ revealed the bone loss by ovx and increased bone density after PTH treatment in femurs. ${ }^{* *} p<0.01$ vs sham, ${ }^{*} p<0.05$ vs OVX. Statistical analysis was performed one way ANOVA followed by Bonferroni's Multiple Comparison Test. Data are depicted as mean \pm SEM, $n=5$ per group. (B) Temporal bioluminescent imaging of Col(I)-Luc mice after ovx and sham and following PTH treatment. Data are collected as a ratio by normalizing average light intensity from the same group at each time point to that at day 1 . Statistical analysis was performed by two-tail paired Student's t-test. * $p<0.05$, ** $p<0.01$, *** $p<0.001$ vs day 1 if there is no indication. Data are depicted as mean $\pm \mathrm{SD}, \mathrm{n}=5$ per group.
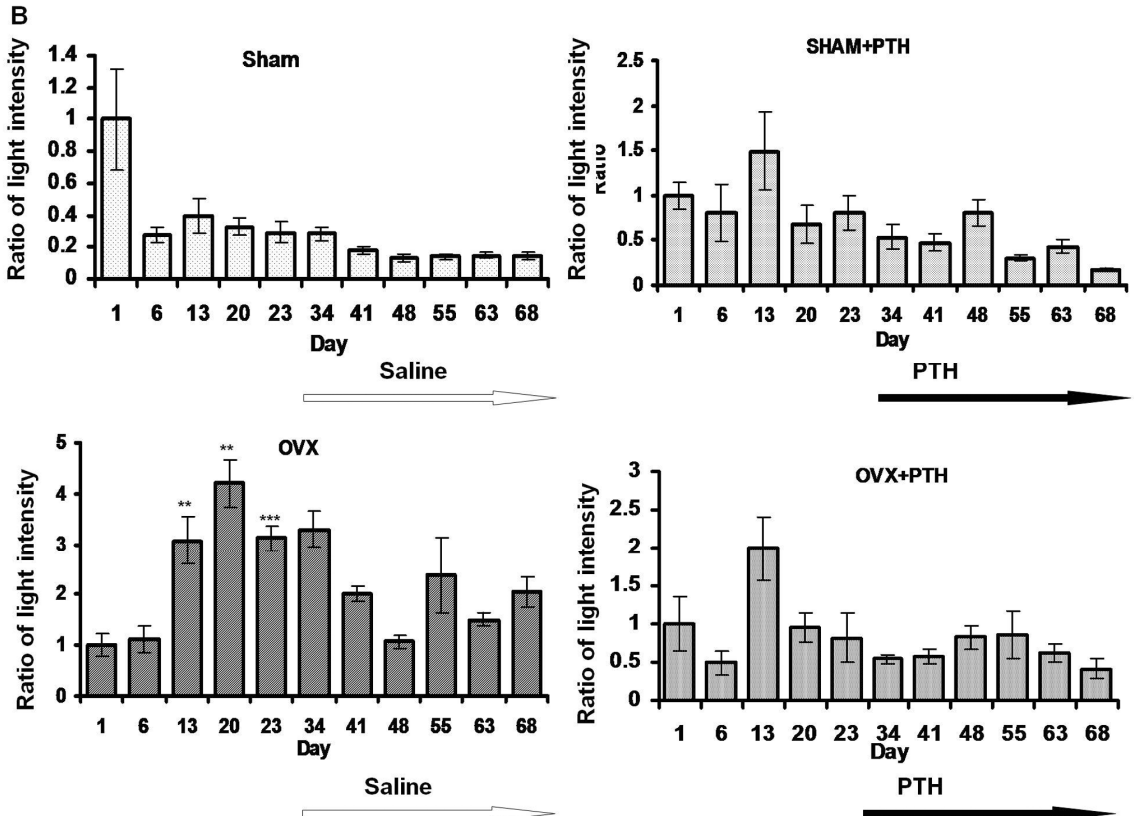

\section{Luciferase activity is upregulated during bone repair}

Luciferase activity from Col(I)-Luc mice was expressed strongest from tails. Individual vertebrae could be clearly distinguished, with less light from 
the smaller vertebrae. During one experiment, one mouse tail tip was accidentally squeezed. Seven days later, we observed stronger light emission at the injured site for 7 weeks with a peak at week 3 compared to control mice (Fig. 5A). To further investigate the bone repair in these mice, we designed a tail fractural model by creating an $\varnothing 1 \mathrm{~mm}$ hole in one vertebra of a mouse tail. BLI was performed for 8 weeks from both the operated group and control group (Fig. 5B). The light intensity from the control group declined gradually within 8 weeks. At first, the loss of a piece of bone in the operated group caused a dramatic drop of light intensity, even below that of the control group. When bone regeneration proceeded, the light intensity increased with a peak at day 20 , and then staying at a plateau until day 48 before it went back to control level at day 56.

\section{Col(I)-Luc mice can be used in investigating ectopic bone formation}

BMP2 is a protein with the potency to induce bone formation at ectopic sites. To assess whether we could detect ectopic bone formation using BLI, we implanted BCP particles coated with rhBMP2 in a subcutaneous pocket in 4 male Col(I)-Luc mice, and performed BLI every week. Two weeks after implantation, light was detected at the site of the rhBMP2 implant in all mice whereas no light was detected from either empty pockets or uncoated BCP controls (Fig. 6A). Light intensity peaked at week 2 and 3, and declined afterwards until week 6 when the light intensity was below detection from 3 mice (Fig. 6A and B). However sustained light was detected from one mouse with an open on rhBMP2 pocket demonstrated that the skin required certain light intensity to penetrate through (Fig. 6B R BMP). After eight weeks postimplantation, scaffolds were explanted. We performed histomorphometry and observed $12 \%$ bone in the BPM group and no bone in the control groups (Fig. $6 \mathrm{C}$ and D). 
A
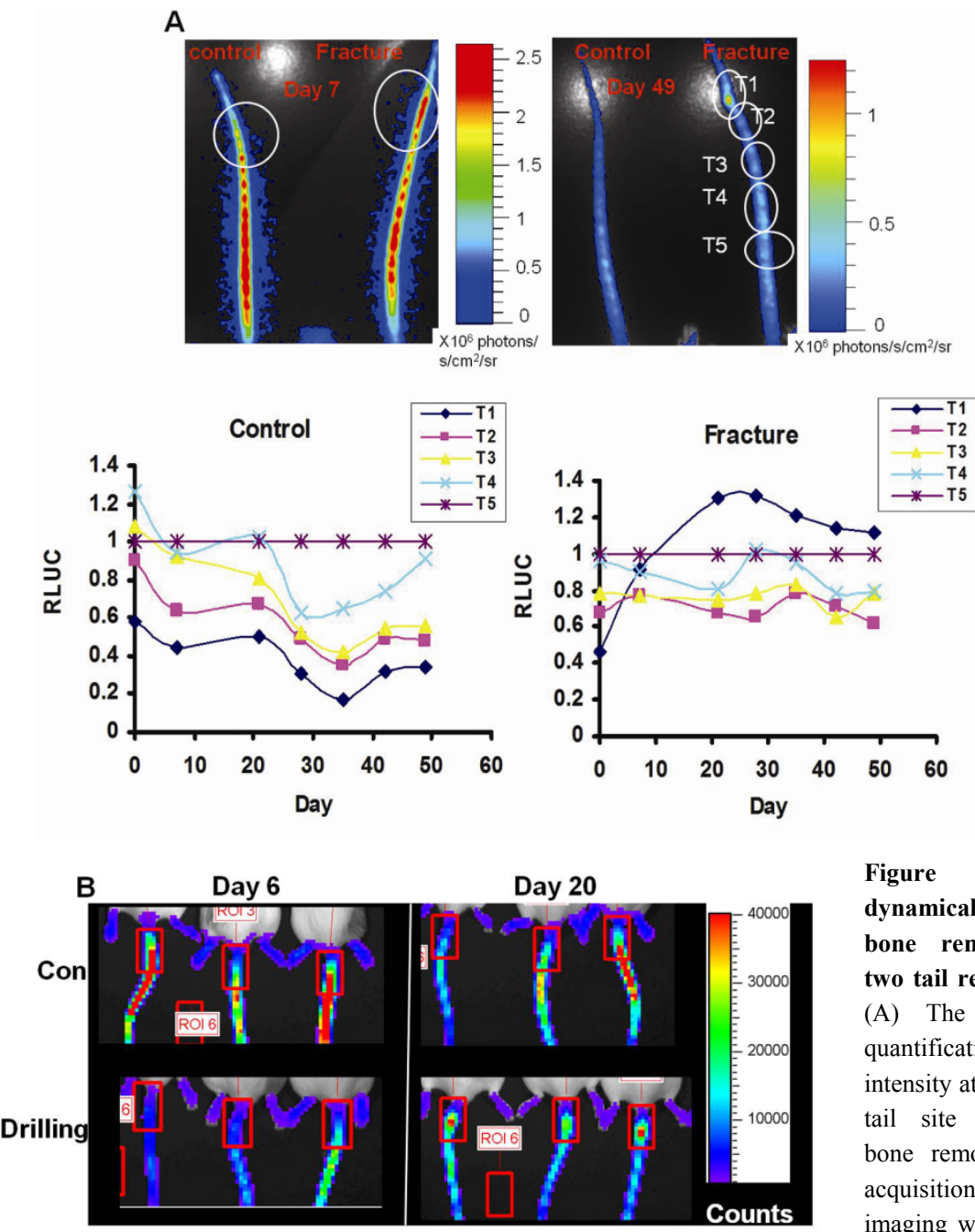

Figure 5. BLI dynamically monitored bone remodelling in two tail repair models. (A) The image and quantification of light intensity at the squeezed tail site (T1) during bone remodelling. The acquisition time for this imaging was 3 minutes.

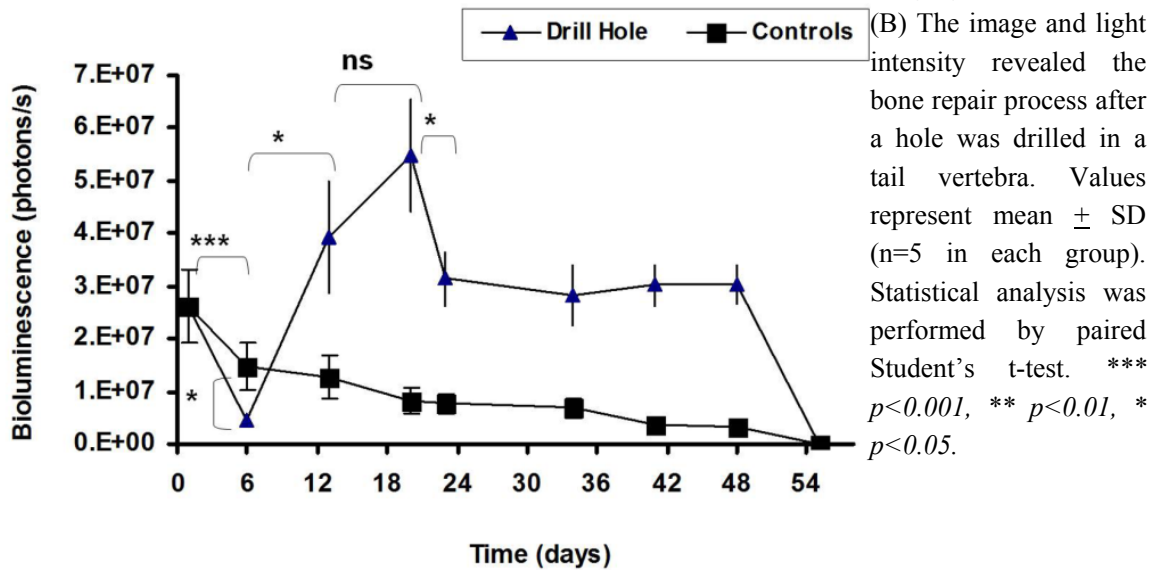




\section{DISCUSSION}

In this manuscript, we used BLI to detect luciferase expression from bony organs of transgenic mice using the -1721 to -1365 promoter region of the pro- $\alpha 1(\mathrm{I})$ collagen gene. To our knowledge, this is the first report of BLI of collagen type I expression in the processes of bone remodelling and formation. Bone is a dynamic tissue, undergoing remodelling throughout life including two processes by two type cells, bone formation by osteoblasts and bone resorption by osteoclasts. Bone formation reaches its peak in childhood, after which homeostasis is maintained up to the time when post-menopausal or senile osteoporosis emerges. Light emission driven by the pro- $\alpha 1$ (I) segment promoter shows high collagen type 1 expression in young male mice, followed by a rapid decrease upon reaching adulthood. Luciferase activity from the jaw did not drop as strikingly as that from the tail and other skeletal sites, which may be explained by the fact that the incisor grows continuously whilst the animal lives.

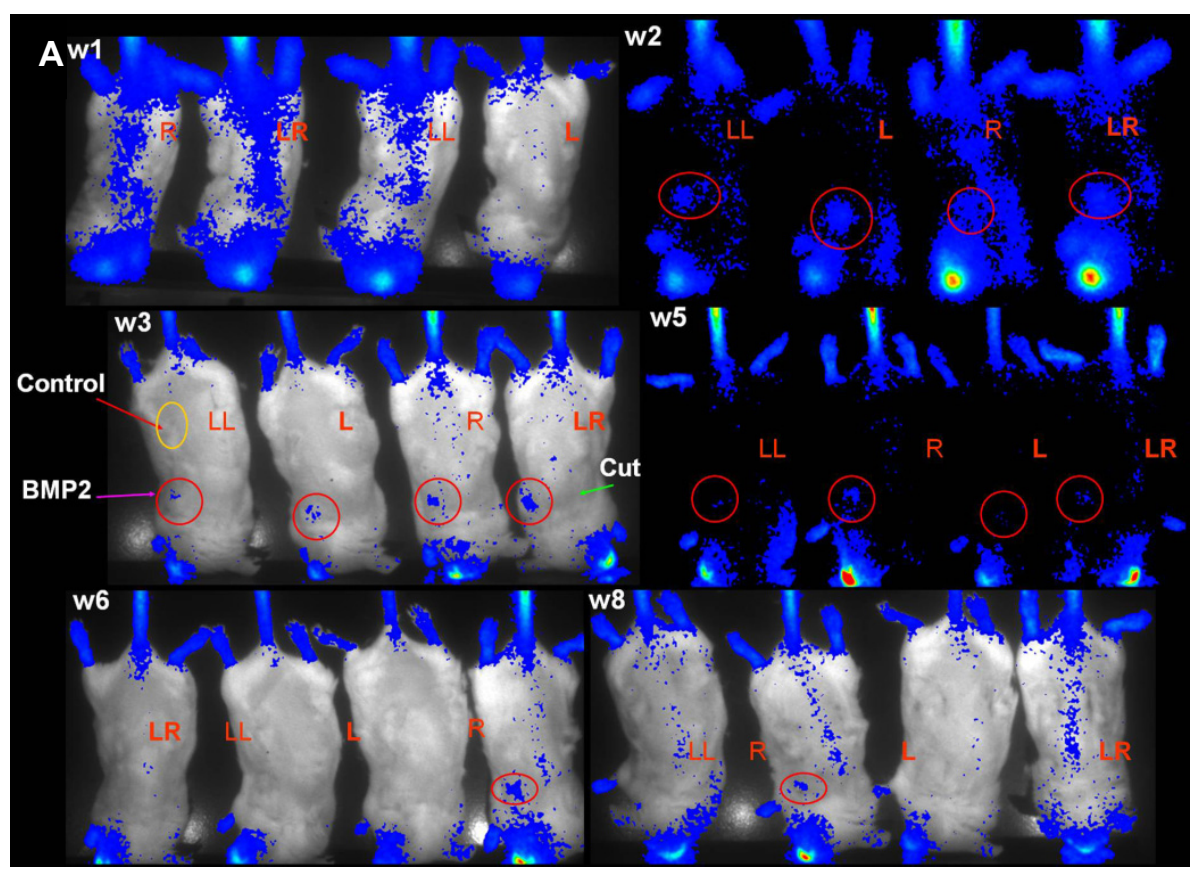



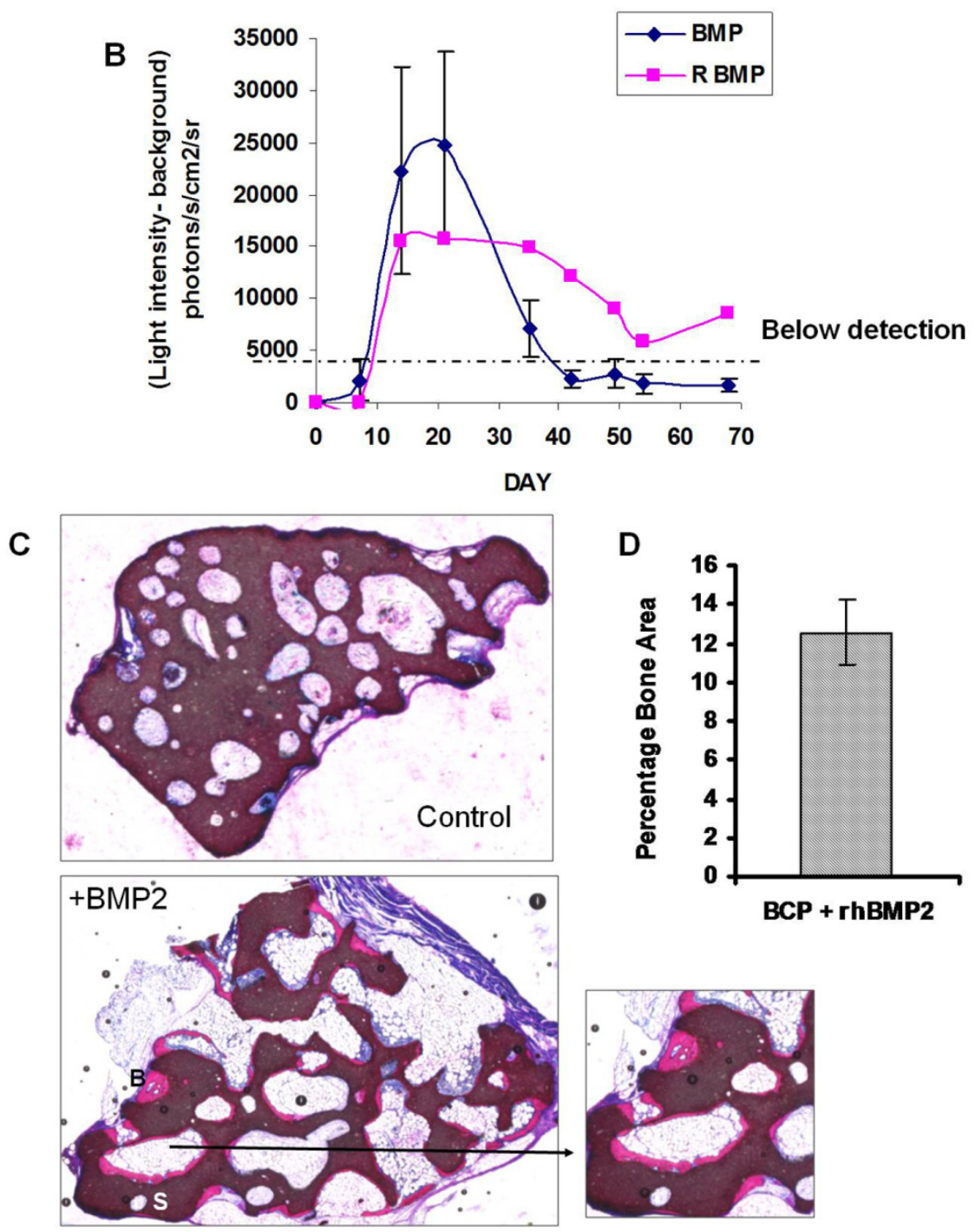

Figure 6. BLI monitored the subcutaneous ectopic bone formation induced by rhBMP2. (A) The bioluminescent images revealed ectopic bone formation. Light was detected from BMP2 pockets from all 4 mice implanted after 2 weeks, kept until week 5, and then diminished afterwards in 3 out of 4 mice, except mouse R. The acquisition time for these images was 3 minutes. Control meant BCP alone indicated by a yellow circle, BMP was BCP with rhBMP2 by a red circle, and cut was a empty pocket. LL, L, R and LR are the ear markers of mice. (B) Light intensity monitored the dynamic development of bone formation. The light intensity of rhBMP2 implant was subtracted by that of BCP implant. Mouse R had an open in rhBMP2 pocket. Values represent mean $\pm \mathrm{SD}(\mathrm{n}=3$, exclude mouse $\mathrm{R})$. (C) Histological staining showed bone distribution. B: bone; S: scaffold. (D) Quantification of the newly formed bone. Values represent mean \pm SD ( $n=3$, exclude mouse R). 
The fact that we did not observe light in the later live of the mouse, even though bone remodelling is a constant process may be because at that stage the cumulative promoter activity was below the detection limit. Tissue particularly attenuates light with a wavelength in the range of 400 to $600 \mathrm{~nm}$, but less between 600 to $950 \mathrm{~nm}$ [192]. Indeed, we observed that (pigmented) hair and skin absorbed much light, as reported [193]. In principle, the light intensity is attenuated approximately 10-fold for every centimetre of tissue depth [194]. New technological developments such as stronger promoters and improved versions of the luciferase gene may be used to induce the level of luciferase activity such that even adult bone remodeling can be detected using this promoter.

A second potential application of the Col(I)-Luc mouse line are their multipotent mesenchymal stem cells, which may be used in bone tissue engineering research for high throughput screening of osteogenic compounds. We used a previously described protocol [189] to isolate mMSCs and succeeded in isolating and culturing mMSCs in vitro. The cells harboured the luciferase gene, exhibited MSC morphology, and differentiated along the adipogenic and osteogenic lineage in vitro. During mineralization in vitro, mineralization typically starts after a collagen matrix has formed, but to our surprise mineralizing Col(I)-Luc mMSCs did not express luciferase. A possible explanation was that the transgene was silenced during in vitro expansion of the cells. Alternatively, the bone-specific enhancer of the collagen type 1 gene is not activated during the osteogenic process of mMSCs.

Next, we anticipated activation of the reporter gene in ovariectomised female mice, because induced bone turn-over has been reported with this procedure. $\mu \mathrm{CT}$ revealed that the femoral trabecular BMD reduced after ovx, and PTH treatment was able to restore it. BLI revealed increased light intensity in one of the two group of ovx mice. In the other group, increased luciferase activity was observed at only one time point. More experiments will have to prove that Col(I)-Luc mice respond to the ovx treatment by upregulation of luciferase activity. In none of the groups we observed increased luciferase activity in response to PTH treatment, even though this had been reported previously using a biochemical luciferase assay [96].

Based on the absence of detectable luciferase activity during adult bone remodelling and an undetectable effect of rhPTH, our following data supported that Col(I)-Luc mice are more suitable for detection of local bone formation. Fracture healing is a complex process in which a cascade of cellular events is 88 
starting with an inflammatory response [195-197]. Next, different cell types are involved, including mesenchymal stem cells, osteoblasts, endothelial cells and osteoclasts, in the processes of proliferation, differentiation, synthesis of extracellular matrix proteins and angiogenesis. Usually, the fracture gap is first bridged by woven bone formed by either endochondral or intramembranous ossification routes, which is ultimately replaced by lamellar bone through bone remodelling. In our fracture model, light intensity reached peaks at around week 2 and 3, and high level expression sustained during the whole repair processes. During normal fracture healing, the expression of bone specific markers reaches maximal levels around day 15 which corresponds well with our findings [198, 199].

Col(I)-Luc mice can be applied in a second local bone formation model: ectopic bone formation. BMP2 is a well known protein in clinical therapy for enhancing bone formation and BMP2 is known to induce ectopic bone formation in subcutaneous or intramuscular implants in mice [200-202]. A lot of research is devoted to improving the dosage and mode of delivery of BMP2. In Col(I)-Luc mice, luciferase activity was detected upon subcutaneous implantation of rhBMP2, which peaked on week 2 for 2 weeks and then declined. Our results suggest that Col(I)-Luc mice are a useful tool to evaluate the issues mentioned and to assess new potential anabolic molecules. It is known that some fluorescent dyes and radiolabeled bisphosphonates are also used as clinical imaging agents for bone scans. It is due to that hydroxyapatite (HA), the major mineral product of osteoblasts, has high affinity to bind with pyrophosphonates, phosphonates, and synthetic bisphosphonates. Xytetracycline and xylenol orange marker were used to label newly formed HA at different time points for evaluating the bone formation dynamics after postmortal fluorescence microscope analysis [203]. Zilberman tracked dynamic bone formation by administration of the fluorescent bisphosphonate imaging agent OsteoSense [204]. In that study, mMSCs transduced with BMP gene were implanted into muscles and fracture site at forelimbs. Fluorescence molecular tomography revealed the appearance of signal with a peak at day 14 when ectopic bone formed in muscles, and a peak of signal at day 21 in the fracture model. However the auto fluorescence from tissues limits this technique's sensitivity and the external light source with high energy can be destructive to animals. But the peak time points he showed corresponded well with this study, which further demonstrated that our system is a sensitive and reliable model to investigate bone remodelling and formation. 
In summary, despite some limitations in detection level and thus applications, our results raise interesting findings with Col(I)-Luc mice. This mouse line is useful to investigate postnatal bone development and repair, and even ectopic bone formation, which serves as a convenient tool to better understand skeletal remodelling processes and to evaluate osteoinductive compounds or scaffolds in vivo in a non-invasive and quantitative way.

\section{Part 2: hOC-Luc mouse}

\section{MATERIALS AND METHODS}

\section{Animals}

Human osteocalcin-luciferase FVB/N transgenic mice were created as previously described [97]. All experiments were approved by the local Ethical Committee for Animal Experimentation and in compliance with the Institutional Guidelines on the use of laboratory animals. The genotyping of transgenic mice was in the same procedure as for Col(I)-Luc mouse.

\section{Imaging surgical effect of hOC-Luc mice}

Eight male mice were subcutaneously implanted with 3 different scaffolds, Ø4mm cylinder-like copolymer of PEGT/PBT 330/55/45 (poly(ethylene glycol)-terephthalate /poly(butylene terephthalate), Polyactive ${ }^{\mathrm{TM}}$, Isotis, S.A.), BCP1150 of 2-3mm in size, BCP1300 of 2-3mm in size (3 BCP: $\beta$-calcium phosphate ceramic particles were taken as one sample) and an empty pocket. The incisions were closed using a clip. In the following 6 weeks, BLI was done with CCCD camera from Ivis (Xenogen) every week. Mice were under anaesthesia by Isofluorane and injected intraperitoneally with $100 \mu \mathrm{l}$ D-luciferin $(25 \mathrm{mg} / \mathrm{ml})$ (Synchem Chemie, Kassel, Germany), and 10 minutes later photons were collected. After 6 weeks the mice were sacrificed using $\mathrm{CO}_{2}$.

\section{Imaging ectopic bone formation and histological analysis}

BCP 1150 of 2-3mm in size incorporated with rhBMP2 was previously demonstrated to induce bone formation subcutaneously in mice from our lab. Nine transgenic male mice were implanted subcutaneously with 3 particles in one pocket and 3 particles soaked with 100ng rhBMP2 per ml BCP particles in another pocket in the same procedure as described above, and the pockets were 
closed with a vicryl 5-0 suture. BLI was performed every week for 4 months. Animals were anaesthetized with ketamine, xylazine and atropine solution (ratio of $1: 1.25: 0.75, \mathrm{KXA}$ ) by an intramuscular injection at $0.2 \mu \mathrm{l} / \mathrm{g}$ body weight. Then mice were injected intraperitoneally with $100 \mu \mathrm{l}$ D-luciferin $(25 \mathrm{mg} / \mathrm{ml})$ (Synchem Chemie, Kassel, Germany). BLI was performed for 30 minutes following luciferin injection with a Roper Bioluminescence Imaging System Princeton Instruments (Trenton, USA). The CCCD camera was LN/CCD1300EB with a 50-mm F1.2 Nikon lens (Roper Scientific). The imaging and analysis were done by Metamorph software (Universal Imaging Corp., West Chester, USA). At end the mice were sacrificed using $\mathrm{CO}_{2}$ and samples were explanted, fixed in $4 \%$ paraformadehyde, dehydrated and embedded in methyl methacrylate (Sigma) for sectioning. Approximately $10 \mu \mathrm{m}$ thick, undecalcified sections were processed on a histological diamond saw (Leica saw microtome cutting system). The sections were stained with basic fuchsin and methylene blue to visualize bone formation in pink color.

\section{RESULTS AND DISCUSSION}

In previous reports, hOC-Luc mice displayed luciferase expression limited to bone tissues. They responded to bone fracture repair and bone formation induced by rAAV-rhBMP-2 virus injection into muscles by showing higher light intensity at the surgical site with a peak at day 6 [86, 87]. As such, hOC-luc mice can potentially be applied to investigate ectopic bone formation. To assess this further, we implanted 3 types of scaffolds: Polyactive ${ }^{\mathrm{TM}}$, BCP1300 and BCP 1150 and we generated an empty subcutaneous pocket as negative control. The latter material has proven osteo-inductive potential in goat and dog muscle tissue and we anticipated to observe ectopic light from the site implanted with BCP1150. As reported before, we observed light from paws, feet, tails and jaws. Large inter-animal heterogeneity was seen in the luciferase expression level (Fig. 1, week 1). Surprisingly, light was detected from all surgical sites within the first week, which gradually declined at week 2 and week 3 (Fig. 1). Apparently, the osteocalcin is activated during skin wound healing. After the skin wound signal declined, no further light was detected from the pockets implanted with biomaterials, including BCP1150. 

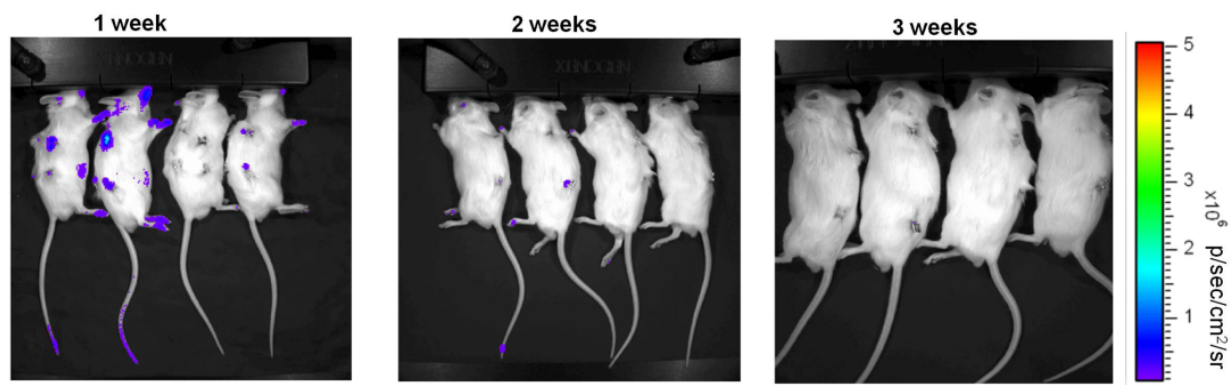

Figure 1. hOC-Luc mice responded to the wound. Three empty scaffolds were implanted subcutaneously in 8 mice, including Polyactive, BCP 1150 and BCP 1300, and an extra empty pocket. BLI was performed for 6 weeks to evaluate the implanted pockets. Light was detected in the first week, then decreased in the next two weeks, and then diminished. The acquisition time for this imaging was 1 minute.

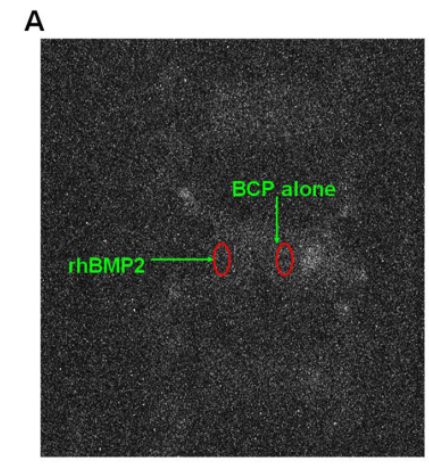

Day 0

B

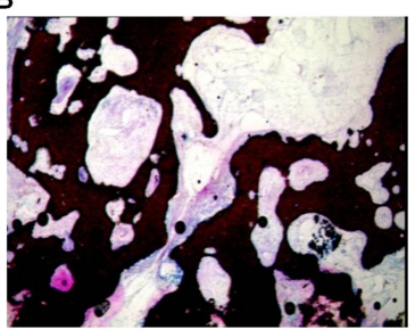

BCP alone

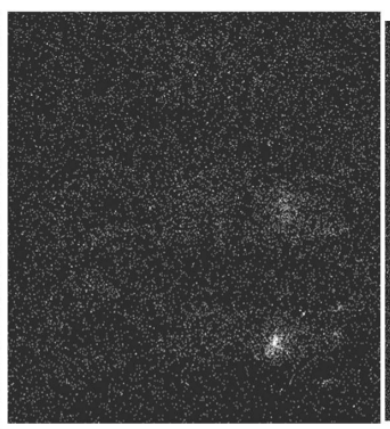

4 weeks

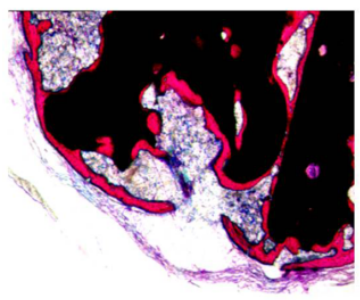

BCP with rhBMP2

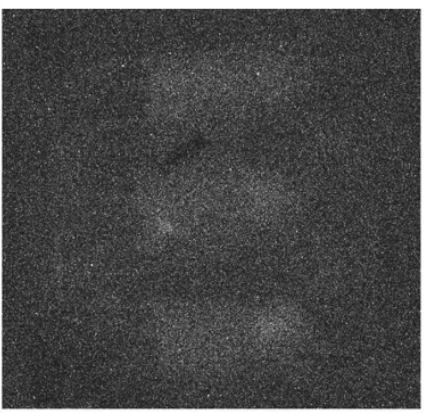

4 months

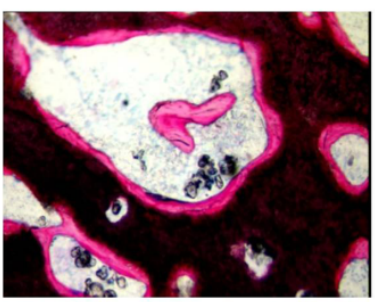

Figure 2. Investigating the ectopic bone formation in hOC-Luc mice by BLI. (A) BLI before implantation and 4 weeks and 4 months afterwards. No signal was detected from the implantation sites. The acquisition time for this imaging was 15 minutes. (B) Histological staining of the implant showed newly formed bone (in pink) in rhBMP2 implant.

In a second study, BCP 1150 with and without rhBMP2 were implanted in hOC-luc mice to assess whether ectopic bone could be monitored. Four months post-implantation, scaffolds were explanted and ectopic bone formed only on scaffolds with rhBMP2 (Fig. 2), to the same extend as seen in the Fig. 6 (part 1). Again, we observed large variation in luciferase expression among 
mice that were positively genotyped for the luciferase gene prior to BLI. Several mice expressed luciferase in the same pattern as in the previous experiment but weaker and previously undetected strong light was detected from cervical vertebrae. Nevertheless, no light was detected from the implantation sites during a period of 4 months in which BLI was performed. The light intensity from newly formed bone in our case might be below the detection for this mouse model, even though it was sufficient to be detected in Col(I)-Luc mice. In addition, the animals themselves had low luciferase expression levels that indicated a low luciferase gene expressing, which might also explain the discrepancy. However, this problem can be solved by enhancing the detection sensitivity of this reporter. Luciferase 2 gene is a new generation of luciferase gene, which has increased reporter protein expression by codon optimization of synthetic genes for mammalian expression. We found that the luciferase 2 gene produced a 10 times higher signal than luciferase under the same condition in chinese hamster ovary cells transfected with hypoxia responsive element (HRE)-luciferase (unpublished data). Thus, the detection sensitivity of hOC-Luc mouse can be improved by replacing with luciferase 2 gene.

\section{ACKNOWLEDGEMENTS}

We wish to thank Dr. Huipin Yuan from University of Twente, Enschede, The Netherlands to kindly provide the BCP scaffolds. We also thank Gerard Geelen and Agnes Goderie from the animal facility (GDL), Utrecht, for excellent animal care. The research is sponsored by a grant from Senter/Novem. 


\section{Chapter 6}

\section{General conclusion, discussion and future perspective}




\section{Tissue engineering is a multidisciplinary field}

Due to their potential to differentiate into several cellular lineages, mesenchymal stem cells (MSCs) are widely attracting the attention of scientists and surgeons to develop clinical application to repair and restore lost and dysfunctional tissues. The development of bone tissue engineering (TE) shifts the focus in orthopaedics from non-degradable bone implants to bone regeneration by osteogenic progenitor cells or mesenchymal stem cells. The bone tissue engineering concept has been successively demonstrated by preclinical research and even clinical trials $[8,9]$. However the current inability to bridge large-size bone defects limits its application. Therefore, improving bone formation in vivo is a bottleneck that we need to overcome. As mentioned previously, bone tissue engineering includes multiple fields of research, and one of the current focuses is on enhancing MSCs proliferation and differentiation using knowledge from biology, material science and the engineering disciplines. For instance, molecular biology approaches bone TE by stimulating MSC proliferation and osteogenic differentiation in vitro, and engineering aims at preparing biomaterial scaffolds or grafts which improve MSC survival and differentiation in vivo. To evaluate the efficiency of these strategies, novel imaging techniques are offering the possibility to monitor cellular behaviour and tissue formation both in vitro and in vivo.

This thesis clearly depicts that cell-based tissue engineering is a multidisciplinary approach. It contains three sub-goals: the first part is to investigate a molecular signalling pathway using a pharmaceutical approach to stimulate hMSCs osteogenic differentiation. The second part is to investigate cell survival and nutrient availability to cells in tissue engineered constructs by optical imaging. In addition, it also provided tools to investigate nutrient diffusion in tissue engineering constructs. The third part aimed at evaluating the application of transgenic mouse lines in assessing bone formation using bioluminescent imaging.

\section{Improving hMSC osteogenic differentiation}

Human mesenchymal stem cells (hMSCs) isolated from bone marrow display the potency of osteogenesis, adipogenesis and chondrogenesis in vitro in particular media. After implantation in vivo, MSCs form bone tissue. However, its rare occurence in bone marrow, its heterogeneous differentiation capacity 
among individual cells and among donors and its fading differentiating capacity during expansion [23], all limit hMSC application in curing clinically relevant critical-sized bone fractures. One approach to have sufficient numbers of cells is to use another tissue source to isolate MSCs. MSCs isolation from muscle and fat has been reported [205], which represents a less invasive procedure and yields a large number of cells. Although adipose-derived stem cells (ADSC) can differentiate into multiple cellular lineages including osteoblasts and chondrocytes in vitro and in vivo, bone marrow-derived MSCs are still attracting most attention. Another approach is to improve hMSCs proliferation without compromising their differentiation ability or to enhance their differentiation capacity. Thus it is crucial to understand the developmental aspects of differentiating a MSC into an osteoblast. In chapter 1, Fig. 4 depicts the molecular and genetic regulators involved in this procedure in detail. Based on abundant biological information, many compounds have been tested for this purpose. For instance, lithium, an activator of Wnt signalling pathway, enhances hMSCs proliferation without affecting hMSCs differentiation capacity [206]. Bone morphogenetic proteins are clinically used to treat osteoporosis or bone fracture and dexamethasone is used to induce osteogenic differentiation in hMSCs. Nevertheless, hMSC differentiation capacity can be further improved by treating with other compounds, such as cAMP, a PKA activator, which is known to enhance hMSC osteogenic differentiation in vitro and in vivo [28]. This in turn indicates that there is still much space to optimize the current culturing protocol by seeking for other compounds with bone anabolic effects.

Chapter 2 depicts the effects of stimulating or inhibiting different protein kinase $\mathrm{C}$ (PKC) isozymes on hMSC osteogenic differentiation. Some reports show that certain $\mathrm{PKC}$ isozymes are involved in osteogenic differentiation or osteoblast proliferation. For instance, $\mathrm{PKC} \delta$ and $\mathrm{PKC} \mu$, are known to be involved in osteogenic differentiation in murine cells and animal models and PKC $\alpha$ has been implicated in human osteoblasts proliferation [98, $100,144]$. We treated hMSCs with PKC activators and inhibitors to investigate cell proliferation and differentiation in vitro, and observed that different PKC subfamilies had opposite roles in ALP expression and mineralization.

(Conclusion ${ }^{1)}$ In conclusion, inhibition of the conventional family of PKCs/PKC $\mu$ stimulates hMSC ALP expression, collagen I expression and mineralization in vitro, whereas inhibition of PKC abolishes osteogenic

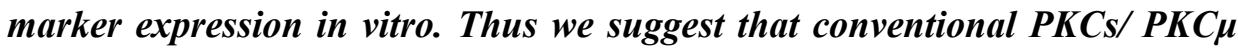


play a negative role and PKC $\delta$ is a pivotal player in osteogenic differentiation of hMSCs.

To elucidate a certain gene's function, the best way is to genetically knockout or knock down the interested gene expression. However, an efficient protocol to perform siRNA with hMSCs is still lacking, and thus our research was based on a pharmaceutical approach. Still, pre-treatment of hMSCs with 100nM Gö6976, thus inhibiting conventional PKCs, did enhance the in vitro osteogenic differentiation, which provided a new cue to be applied further in developing bone TE strategies.

Often, scientists use murine cell lines such as $\mathrm{C} 2 \mathrm{C} 12$ and MC3T3 to screen genetic and molecular mechanisms, due to their controllable culture and consistent performance, thus representing clinical models. In chapter 2 , a contradictory response between murine cell lines and hMSCs to PMA is noted (data not shown), where MC3T3 and $\mathrm{C} 2 \mathrm{C} 12$ cell proliferation was up-regulated by PMA. Clearly this demonstrated a discrepancy between murine cell lines and hMSCs, which was also reported by Dr. Siddappa on the effect of PKA signalling pathway in the osteogenic differentiation of hMSCs and murine bone marrow stem cells [28, 207].

(Conclusion 2) hMSCs are the most reliable cell type to screen novel genes or molecules for clinical application.

In the future, in order to elucidate clearly the effects of different PKC izozymes in hMSCs, siRNA interference in hMSCs is recommended.

\section{Investigating nutrient availability to cells}

In order to implant cells in vivo, biomaterial scaffolds are required to support and locate cells. The scaffolds comply with some fundamental properties such as biocompatibility and proper pore size and porosity to allow blood vessel and tissue infiltration. It has been reported that cells located deeper than $200 \mu \mathrm{m}$ in the scaffold can not survive during conventional in vitro static culture and in vivo implantation due to limited nutrient diffusion. Therefore, scaffolds with a clinically relevant size (several hundred micrometers) displayed heterogeneous cell/tissue distribution, proliferation and even differentiation, which is also a critical factor to determine the success of a graft. Improving nutrient availability to cells in three-dimensional constructs is one of the biggest challenges in current tissue engineering research. An efficient evaluation method is required in order to study cell survival and nutrient availability to 
cells on scaffolds. Chapter 3 and chapter 4 describe the use of transgenic cell lines to investigate the above issues by optical imaging.

In chapter 3, a transgene containing a hypoxia-responsive element driving luciferase was introduced into Chinese hamster ovary cells and in chapter 4, green fluorescent protein (GFP) replaced luciferase in the construct. Among many nutrients, oxygen is thought to be critical, due to its low solubility and high consumption rate. Chinese hamster ovary cells have a high metabolic activity, with an approximately 30 times higher oxygen consumption rate than hMSCs $\left(\mathrm{O}_{2}\right.$ consumption rate for $\mathrm{CHO}: 3.2-1.8 \times 10^{-13} \mathrm{~mol} \mathrm{O}_{2} / \mathrm{cell} / \mathrm{h} ; \mathrm{O}_{2}$ consumption rate for hMSCs in 3D scaffolds in bioreactors: $1.2-0.17 \times 10^{-14} \mathrm{~mol}$ $\mathrm{O}_{2} /$ cell/h) [208, 209]. Thus, we first assessed if this cell line could act as a model to study nutrient availability in TE both in vitro and in vivo. When cells are in a low $\mathrm{O}_{2}$ environment, hypoxia inducible factor 1 (HIF1) accumulates and binds to the hypoxia responsive element (HRE), thus leading to the transcription of luciferase or GFP.

HRE-Luc CHO (HL) cells expressed lucifease in 2D and 3D culture conditions as a response to decreased $\mathrm{O}_{2}$ level in the media. Chapter 3 describes for the first time direct evidence that perfused bioreactors provide better nutrient delivery than 2D static culture, illustrated by a higher luciferase activity in the latter. In addition, HL cells also showed upregulated luciferase activity within constructs further away from the nutrient source. Furthermore, bioluminescent imaging of a 3D hydrogel containing HL cells revealed strong light emission. When HL cells were implanted subcutaneously in nude mice, we detected increased luciferase expression over time. Oxygen level was monitored in an individual cell using GFP as a reporter. When HRE-GFP cells were incubated in a 3D hydrogel under static culture, cells expressed GFP and an oxygen diffusion limitation was evident from around $200 \mu \mathrm{m}$ after one day.

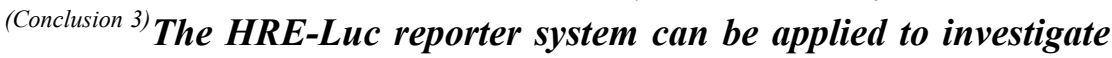
$\mathrm{O}_{2} /$ nutrient availability in the context of tissue engineering.

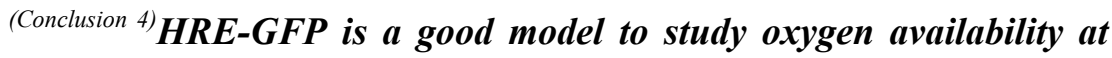
the single cell level.

Optical imaging using firefly luciferase or GFP is a direct and efficient tool to study gene expression and function. It is limited to pre-clinical research because it is based on transgenesis. The promoter can be tailored easily, but luciferase activity is determined not only by cell number and promoter activity but also by luciferin concentration. When luciferin availability is the ratelimiting factor, the hypoxia status is underestimated when an HRE-Luc 
construct is applied. (Conclusion ${ }^{5)}$ The HRE-Luc construct can be applied to investigate hypoxia/nutrient status in different constructs only when they have same luciferin diffusion property. For materials with different diffusion properties, the HRE-GFP (HG) reporter system is recommended. Without the extra requirement of adding luciferin, the cells expressing GFP can be detected by fluorescent microscopy. In chapter 4, agarose gels had more GFP positive cells with prolonged incubation.

Within the limits, our results show that the HRE-reporter system can provide very precious information on scaffold architecture design in order to improve nutrient delivery within TE constructs. For instance, adjusting the tortuosity or pore size or channel width of scaffolds might affect medium delivery, which can be monitored using this reporter system.

When cells are hypoxic, the HIF1 signalling pathway is activated, resulting in activation of downstream target genes such as VEGF, a key factor for recruiting cells to build the vasculature network. Hence, angiogenesis or vascularisation starts. Luciferase expression or activity in this construct can be an indicator of VEGF production. Furthermore, in a natural physiological environment, the $\mathrm{O}_{2}$ level is low in bone marrow; therefore, hypoxia has impact on cell proliferation or differentiation. Many reports state that temporary hypoxia treatment improves graft survival after implantation in vivo [56], although it might hamper osteogenic differentiation. It was reported that $3 \% \mathrm{O}_{2}$ has a negative effect on osteogenesis, but has a positive effect on prolonging the lifespan and maintaining the stemness of hMSCs [210]. However these findings are contradicting other studies where rat MSCs cultured at 5\% $\mathrm{O}_{2}$ had a better bone forming performance compared to rat MSCs grown at 20\% [211]. It seems that the acute oxygen level is essential to decide the cell fate. It is noted that in chapter 3 Fig. 3, the HRE-Luc reporter accurately reflects $\mathrm{O}_{2}$ level in both $20 \%$ and 5\% bioreactors. Therefore, HRE-reporter system can serve as an $\mathrm{O}_{2}$ meter for monitoring cell differentiation.

As mentioned before, optical imaging with a reporter gene is a very versatile tool to monitor gene and protein expression because reporter expression can represent virtually any interesting gene. In this thesis, I have used two promoters, HRE and CMV. We already discussed HRE extensively. The CMV promoter is constitutively active and luciferase expression or activity represents cell number and luciferin availability. In chapter 3, the CMV-Luc cell line was used as an index for cell number in an in vivo study, where 2 out of 3 mice showed a declining light intensity pattern over time indicating cell death 100 
(data not shown). In chapter 4, the same cell line was used to study luciferin diffusion in biomaterials. The images showed clearly the dynamic process of luciferin influx indicated by the progressively increasing light intensity through the agarose gel (Fig. 4). (Conclusion 6) The slope of the dynamic light intensity curve of CMV-Luc reflects luciferin diffusion in agarose gels, and provides information on molecular diffusion in the molecular size range of luciferin (molecular weight 280Dalton).

Again, we are stressing that this technique can be tailored to many interesting proteins by using its corresponding regulatory sequence. For instance, we can investigate BMP presence or activity in a local release profile strategy of biomaterials by using BRE-Luc (BMP-responsive element) transgenic cell lines [84]. Thus, transgenes obtained through biological research can be applied to investigate cell response to the environment/scaffold, which in turn unravels scaffolds properties.

\section{Monitoring molecular diffusion of nutrients}

In chapter 3 and 4 , we have demonstrated that cell-based constructs have limited nutrient diffusion in vitro and in vivo. In the natural physiological environment, molecules diffuse locally from adjacent blood vessels, and the diffusion rate also depends on the presence of extracellular matrix (ECM) proteins. A lot of effort has been made to adjust porosity, permeability and architecture of scaffolds to improve nutrient and waste diffusion. Still, it is necessary to monitor molecular mobility within a certain environment, because it can be very informative for biomaterial design and manufacturing. We used a transgenic cell line to study oxygen and luciferin availability in cells and diffusion based on cellular responses in chapter 3 and 4 . Next, we have studied molecular mobility directly by fluorescent recovery after photobleaching (FRAP). FRAP dynamically monitors fluorescence intensity recovery by diffusion or molecular movement into a photo-bleached area. The diffusion coefficient can be calculated according to the half time of recovery. In our studies, we used FITC labelled-dextrans with different molecular weights $(0.389,3,10$ and $70 \mathrm{kD})$ as model molecules to investigate their movement in agarose gels. We clearly observed that the diffusion coefficient (D) is dependent on agarose gel concentration and molecular weight but independent on molecular concentration which clearly shows that diffusion is a property determined by the properties of the molecule and the material. ${ }^{\text {(Conclusion 7) }}$ FRAP 
is an efficient and accurate method to assess the diffusion rate of nutrient molecules. With current confocal fluorescent microcopes, FRAP is limited to relatively big molecules, because it was noted that small dyes $(0.389$ and $3 \mathrm{kD})$ had an inefficient fluorescent bleaching, thus less accurate curve-fits and D. The reason can be the long scanning time $0.985 \mathrm{sec}$ ) for each image and the long bleaching time (150 iterations for 30.4 seconds). In the future, a laser source with higher power should be adopted.

The set-up in chapter 4 with agarose gels is a simplified model for real tissues or tissue-engineered grafts where both cells and a cell-secreted protein matrix can occlude the diffusion path leading to a decreased diffusion. There are some studies performed in articular cartilage which is an avascular tissue. Torzilli et al. have shown that the proteoglycans hinder molecular diffusion of $70 \mathrm{kDa}$ dextran and insulin [212]. Leddy and Guilak then observed that dextrans diffusion differed between the zones in cartilage, each with a unique composition with respect to cell number and ECM composition [183]. Other parameters which should be taken into consideration in future experiments are route of entry, affinity to ECM and biomaterials and the charge property which will contribute to the diffusion rate as well. It is reported that positively charged sodium ions diffuse more slowly than negatively charged chloride ions, probably due to interaction with the negative charges on proteoglycans [213]. Furthermore, the dextran molecules used in our study have a size range of physiologically relevant growth factors or molecules, but they have a linear structure which makes them different from proteins, which usually have a secondary structure. In line with this, it was reported that flexible macromolecules have greater mobility than similarly sized globular molecules in a random fiber matrix [214].

FRAP successfully brings insight in molecular mobility in tissues in a direct, efficient, reliable and versatile manner compared to other methods. In the future, we are going to investigate the effect of ECM proteins on dextran diffusion. FRAP provides a tool to investigate biomaterial or tissue diffusion property, but it can also be applied in disease diagnosis. For instance, FRAP has been used in other types of tissues including tumors, where it was observed that molecules diffused 3 orders of magnitute faster in tumor tissue than in cartilage [215]. In another case, when the ECM changed due to age or disease, diffusion differences were detected by FRAP. We again conclude that optical imaging is not only useful to visualize cells but also to study cellular response to materials and material properties. 


\section{Luciferase transgenic mouse in bone tissue engineering}

In chapter 3 and 4, we have used bioluminescent imaging (BLI) of luciferase activity to study gene expression (e.g. VEGF) in cells. When transgenic cells with luciferase were incubated in scaffolds and implanted in vivo in a nude mouse, BLI clearly showed the advantages of being sensitive, real-time, longitudinal, quantitative and non-invasive as compared to the traditionally post-mortem histomorphometry or enzymatic analysis. Especially in the in vivo study, each mouse has its internal control, thus eliminating individual variation and leading to a more reliable result. Stepping further, in chapter 5 we have evaluated two luciferase transgenic mouse strains and assessed the possibility to apply them in the bone tissue engineering.

Transgenic mouse models are widely applied to study gene function. Luciferase transgenesis provides a new application by recording the light intensity after injecting the substrate, luciferin. In chapter 5 , luciferase gene expression was driven by two bone specific protein promoter sequences (derived from the mouse collagen type I and human osteocalcin genes respectively), and the transgenic mice expressed luciferase in bony tissues. Col(I)-Luc mice showed a supreme potential application in monitoring bone formation. During bone repair and remodelling after fracture or bone loss in tails, BLI of Col(I)-Luc mice dynamically monitored upregulated luciferase expression indicating ongoing ostogenesis. Moreover, when ectopic bone formation was induced subcutaneously by implanting rhBMP2 protein, bioluminescence was detected starting from week 2 post-implantation indicating the initiation of bone formation. It is noted that only 4 mice were used in the latter study, which indicates another advantage: reduction of animal numbers. (Conclusion 8) BLI of Col(I)-Luc mice could dynamically track local bone formation. As for hOC-Luc mice, they displayed weak luciferase expression with large inter-animal variability and no response could be monitored to newly formed bone by rhBMP2 implants.

Luciferase activity from the mouse tail did not accurately reflect bone remodelling induced by ovariectomy and PTH treatment in Col(I)-Luc mice. After ovariectomy, half of the group showed significantly upregulated luciferase activity, whereas the other half did not. In the case of PTH treatment we did observe the anabolic effect by $\mu \mathrm{CT}$ scanning, but there was no corresponding

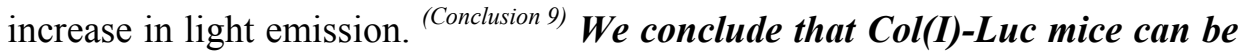
used as an in vivo model to screen for new bone anabolic compounds and to 
investigate controlled release of these compounds in the context of ectopic bone formation.

Chapter 3, 4 and 5 demonstrate the successful application of optical imaging by bioluminescence or fluorescence in a broad context of tissue engineering ranging from cells and materials to animal models. When gene expression is evaluated based on light intensity, the cell number should be corrected for, which is why we included the CMV-Luc cell line in the in vivo implantation study in chapter 3 . It points out that there is a need to create multimodality reporters in one construct. For instance, gene A-luciferase-gene BGFP, or firefly luciferase-renilla luciferase, or different luciferase segments having different $\lambda_{\max }$ should be combined. For in vivo application, the light detection is limited by depth of implant, because skin and tissues do absorb light [194]. Fluorescence tomography (FMT) or bioluminescence tomography (BMT) can be employed to enhance the light detection sensitivity and it can reconstruct a $3 \mathrm{D}$ image thus providing comprehensive information on spatial tissue development. In chapter 5, we have used both BLI and $\mu \mathrm{CT}$ to detect bone formation. In the future the combination of multi-modality imaging techniques will give more accurate results. 


\section{Summary}

Though a new discipline, tissue engineering (TE) has recorded tremendous growth in the past twenty years. The aim of tissue engineering is to repair and regenerate tissues when the body's intrinsic repair mechanisms cannot fulfill the job due to disease or trauma damage. The final goal is to translate the lab research into clinical applications, i.e. "bench to bed" approach. This field covers many different scientific areas including biology, medicine, materials and engineering. Of all the tissues that can be engineered, bone is of our main interest. The bone substitutes have developed from alloys for mere mechanical support to functional autogenetic bone tissues. The concept of bone $\mathrm{TE}$ is to expand and commit bone progenitor cells on certain 3 dimensional (3D) scaffolds for in vivo application. Although bone formation has been demonstrated successfully, there is still more scope to improve the performance of cells to generate greater amounts of bone. This relies on molecules to improve cell behavior including survival, proliferation and differentiation and to make cells as a source of growth factors for local cells.

This thesis contains two parts. The first part is to study the effect of a signaling pathway of protein kinase $\mathrm{C}$ (PKC) on human mesenchymal stem cells (hMSCs) commitment to osteogenesis (chapter 2). The second part is to apply optical imaging techniques to investigate the cellular nutrient level and cell fate both in vitro and in vivo (chapter 3-5).

As mentioned in the introduction of chapter 1, the first step in bone TE is to isolate, expand and differentiate hMSCs in vitro. Due to the low number and limited spontaneous differentiation capacity of hMSCs, molecular cues are required in order to optimize the culture protocol. Literature evidence shows that, PKC family impinges on osteoblasts proliferation and osteogenic differentiation, in multiple cell lines and also the mouse model. So I have evaluated PKC activation and inhibition on hMSCs proliferation and osteogenic differentiation in a pharmaceutical way in chapter 2 . We didn't find the positive effect of PKC activation and inhibition on hMSCs proliferation, but we observed different PKC isozymes working differently for osteogenic differentiation. Amongst the PKC isozymes, $\mathrm{PKC} \delta$ inhibition diminished the osteogenesis of hMSCs, while inhibiting conventional PKC and $\mathrm{PKC} \mu$ enhanced the alkaline phosphatase (ALP) activity and mineralization. Thus the 
positive role of $\mathrm{PKC} \delta$ and negative role of conventional $\mathrm{PKC}$ and $\mathrm{PKC} \mu$ are suggested in order to benefit bone TE.

The second step in bone TE is to culture cells on 3D scaffolds. The current hurdles in 3D cultures is to efficiently provide nutrients to cells whose survival and distribution rely on nutrient availability. In chapter 3 I have evaluated bioluminescent imaging (BLI) in investigating the cell response to surrounding oxygen levels in 3D constructs both in vitro and in vivo. Oxygen was taken as an interesting molecule representing the overall nutrients in the environment, and therefore a gene fragment of hypoxia responsive element (HRE) was used as a promoter driving luciferase gene in chinese hamster ovary cells $(\mathrm{CHO})$. The protein luciferase activity can be evaluated in term of the light intensity emitted when substrate luciferin is added, which is quantified by an enzymatic assay or a CCCD camera. With this reporter construct, limited oxygen availability was recognized in $3 \mathrm{D}$ constructs in static culture but not in a dynamic perfused bioreactor. For the first time, this is a direct proof for beneficially using bioreactors as culture system. In addition, gradually progressed oxygen diffusion was observed in a mimic 3D graft by stacking membrane sheets with cells, layer by layer. Moreover, the non-invasive property enables BLI to evaluate the same sample at multi time points. Thus I observed that cells were in lack of oxygen (or nutrients) and died after being implanted subcutaneously in nude mice. This stressed the necessity to have an instant vascular network in order to maintain cell survival. Stepping further, a limitation distance for oxygen diffusion was recognized at $100-200 \mu \mathrm{m}$ far from the nutrient source by HRE-green fluorescent cells (GFP) in 3D gel construct in chapter 4 .

Moreover, the molecular diffusion in hydrogels was studied in chapter 4 using both BLI of promoter-luciferase cells and fluorescence recovery after photobleaching (FRAP) of fluorescent molecules. I observed and analyzed that molecular diffusion depended both on the molecular size and the gel concentrations. Thus optical imaging in tissue engineering exceeds merely visualizing cells or differentiation. FRAP could provide direct knowledge on nutrient diffusion in biomaterials. Combined with appropriate cellular assays, it can also be a very useful tool to evaluate biomaterial properties to optimize scaffold structural design in order to improve the nutrient or growth factors provision to cells.

Furthermore, BLI extended to the third step in bone TE, in vivo animal models, in which luciferase gene was under control of promoters for bone 106 
specific markers. With bone specific collagen I as the promoter, the Col(I)-Luc mouse line expressed strong light in osseous tissues. Bone repairing during fracture or injury induced strong light at wound sites. When rhBMP2 was loaded in vivo, ectopic bone formation was monitored by tracking the light intensity. Using the same mouse as an internal control renders BLI another merit: reduced animal numbers required for statistics. Thus this mouse line has a lot of potential as it can be applied to study bone remodeling processes and the osteoinductive molecules and scaffolds in bone TE.

Finally, chapter 6 summarizes and discusses the results in this thesis and raises ideas for the future work. Again in conclusion, I have successfully demonstrated the application in bioluminescent imaging in bone TE from both cell and animal levels. 


\section{内容简介}

组织工程作为一个新兴学科在近二十年内取得了突飞猛进的发展。它旨 在修复和重建由疾病和创伤造成损害而且不能自愈的器官组织, 最终实现将 基础研究临床化。组织工程涵盖了不同方面的科学领域, 包括: 生物学, 医 学, 材料科学和工程学。在可构建的组织器官中, 本试验室的研究重点是骨 组织。骨替代品由最初的只是提供承重的合金材料逐步发展到今天的功能性 的自体骨。骨组织工程的概念是获取, 培养骨种子细胞, 在结合一定的三维 支架后被移植到体内。尽管这个概念已经被成功证明, 但是怎样提升骨生成 量还有待进一步研究。然而这个研究涉及到提升移植细胞的成活率, 繁殖和 分化能力, 以及邻近分泌作用, 即通过移植细胞来吸引体内细胞的迁移。

本论文包含两个方面的研究。第一部分是探讨蛋白激酶 C 信号通路对人 体间叶干细胞向骨分化的影响（第二章）。第二部分是结合光学成像技术来 研究体内和体外的细胞营养水平和细胞命运（第三章至第五章）。

在第一章的介绍中提到, 骨组织工程的第一步是提取, 体外增殖和分化 人体间叶干细胞。由于人体间叶干细胞量少和有限的自身分化能力, 于是这 种干细胞的培养方式需要进一步的优化。有文献报道, 通过一些细胞系甚至 动物模型证明 PKC 信号通路对成骨细胞的繁殖和分化有促进作用。于是在本 论文第二章中，通过用一些化学药品来激化或者抑制 PKC，进而调查它对人 体间叶干细胞的繁殖和分化的影响。首先激化和抑制 PKC 对干细胞的繁殖没 有促进作用。但是我们发现不同 PKC 的同功酶对成骨分化有不同的效用。在 PKC 家族中，抑制 PKC $\delta$ 即消除了人体间叶干细胞的成骨性。当抑制了传统 PKC 和 PKC $\mu$ 时, 干细胞的碱性磷酸酶的活性和钙化能力得到了提高。因此我 们建议 PKC $\delta$ 具有促进成骨作用, 而传统 PKC 和 PKC $\mu$ 则有反作用。PKC 家族 以上的特性, 对骨组织工程的进一步研究有着非常积极的作用。于是利用这 个发现有益于骨组织工程, 这一点我们正在做进一步验证。

骨组织工程的第二步是将细胞培养在三维支架上。因为细胞在三维支架 上的成活和分步主要依赖于存在的营养分子, 所以目前在三维支架培养中的 瓶颈是有效的给细胞提供给养。在第三章中, 测试了用生物发光成像来研究 细胞在体内和体外对周围氧含量的反应。我们将氧气作为研究对象, 用它代 表所有营养成分。我们用缺氧反应元件 (HRE) - 荧光素酶作为报告基因, 来标 记中国仓鼠卵巢细胞 (CHO)。荧光素酶在 ATP 和氧气存在的条件下, 与外源 注入的特异底物反应产生发光现象。使用光度计和超低温下高度灵敏的 CCD 相机, 光的强度可以被精确的观测和记录。我们观察到, 三维支架一细胞复合 
体在静态培养时发出了光, 但是在动态生物反应器培养下却没有荧光素酶的 表达。这证明了在静态下, 细胞在三维空间中没有得到足够的氧气（和其他 的营养)。这是第一次为 “使用生物反应器可以提高营养的扩散分部 “这一 理论提供了直接的证据。另外, 根据光强的不同, 我们还发现了在三维空间 复合体内, 氧气分布的不均衡。生物发光成像是一种非损伤的技术, 因此我 们可以多次观察同一被标记的样品。当我们把被标记的细胞移植到裸鼠皮下 后, 我们观察到了缺氧现象, 并且伴有细胞死亡。这一点说明血管网的即时 构建对提高体内移植细胞的成活率是很重要的。更进一步, 当我们用绿色荧 光蛋白代替荧光素酶时, 三维复合体内的临界氧气距离被确定在距营养液 100-200 $\mu \mathrm{m}$ 处（第四章）。

在第四章中，我们用生物发光成像（BLI）和荧光漂白后重建 (FRAP) 技术来调查分子在水凝胶中的扩散效应, 发现分子扩散取决于分子大小和凝 胶浓度。由此, 光学成像在组织工程中的应用不仅只局限于细胞的可视化。 FRAP 可以直接的提供有关营养分子在生物材料中扩散的信息, 同时与其他细 胞性能分析手段结合, 可以用于检测生物材料的性能，从而优化支架结构的 设计, 进而提高营养分子和生长因子对细胞的供给。

接下来, 我们将 BLI 技术拓展到骨组织工程的第三阶段一动物体内, 此时荧光素酶在骨特定蛋白标记控制下。第五章介绍了骨胶原一荧光素酶转基 因老鼠在骨组织处发出强光的现; 此外在由骨折或受伤引起的骨修复过程中, 受伤部位也发出了强光。当骨形态发生蛋白-2 (BMP2) 引入这类老鼠皮下后, 根据光的强度可以追踪异位骨的形成过程。BLI 的另一个优点是使用同一个 动物样品作内部参照, 由此可以减少统计学上所需要的动物数量。综上所述, 这个转基因老鼠可以用于研究骨改造过程, 骨诱导分子和骨诱导支架。

最后, 第六章对本论文所得的实验结果进行了总结和讨论, 并对未 来工作进行了展望。再次总结, 我们从细胞层次和动物模型层次成功的证明 了生物发光技术在骨组织工程中的应用。 


\section{Samenvatting}

Gedurende de afgelopen 20 jaar heeft het de weefselregeneratie een enorme groei doorgemaakt, zelfs terwijl het een vrije nieuwe discipline is. Het doel van weefselregeneratie is het herstel en de regeneratie van weefsel waar de intrinsieke herstelmechanismes van het lichaam dit niet aankunnen als gevolg van ziekte of trauma. Het uiteindelijke doel is het vertalen van experimenteel onderzoek naar klinische toepassingen, een zogenoemde 'bench-to-bed' aanpak. Weefselregeneratie omvat diverse wetenschappelijke gebieden zoals biologie, geneeskunde, materiaalkunde en techniek. Van alle verschillende weefsels waar inmiddels aan wordt gewerkt heeft bot onze hoofdinteresse. Vervangingen voor bot zijn ontwikkeld van metalen, enkel voor mechanische ondersteuning, tot functionele autogenetische botweefsels. Het concept van botweefselregeneratie bestaat uit het expanderen en differentiëren van bot voorloper cellen op 3 dimensionale (3D) scaffolds voor in vivo toepassingen. Hoewel succesvolle botformatie volgens dit concept al is laten zien, is er nog steeds vraag naar verbetering van deze cellen om grotere hoeveelheden bot te genereren. Dit bestaat vaak uit behandeling van cellen met moleculen om de groei, proliferatie en differentiatie te verbeteren en om van deze cellen een bron van groeifactoren te maken voor locale cellen.

Dit proefschrift bestaat uit twee delen. Het eerste deel bestudeert het effect van een signaaltransductieroute, protein kinase C (PKC) op de osteogene differentiatie van humane mesenchymale stamcellen (hMSCs) (hoofdstuk 2). In het tweede deel worden optische beeld technieken toegepast om het cellulaire voedingsniveau en de differentiatie van cellen zowel in vitro als in vivo te onderzoeken (hoofdstuk 3-5).

Zoals uitgelegd in de inleiding van hoofdstuk 1, is de eerste stap van botweefselregeneratie het isoleren, expanderen en differentiëren van hMSCs in vitro. Door het lage aantal hMSCs en de beperkte differentiatie capaciteit van deze cellen, zijn er speciale stoffen in het kweekmedium nodig om het kweekprotocol te optimaliseren. De literatuur laat zien dat de PKC familie inspringt op de proliferatie en differentiatie van osteoblasten in verschillende cellijnen en de muis. Dus heb ik hier het effect van PKC activatie en remming op de proliferatie en osteogene differentiatie van hMSCs geëvalueerd. We hebben geen positief effect gevonden op proliferatie van hMSCs, maar we hebben wel verschillende PKC isozymen gevonden die een verschillend effect 
hebben op osteogene differentiatie. Remming van $\mathrm{PKC} \delta$, remde osteogenese van hMSCs, terwijl remming van conventioneel $\mathrm{PKC}$ en $\mathrm{PKC} \mu$ de expressie van alkaline phosphatase (ALP) en mineralisatie verhoogde. Een positieve rol voor PKC $\delta$ en een negatieve rol voor conventioneel PKC en $\mathrm{PKC} \mu$ worden dus aangeraden om bot weefsel regeneratie positief te beïnvloeden.

De tweede stap in bot weefselregeneratie is het kweken van cellen op 3D scaffolds. Eén van de problemen momenteel is het efficiënt aandragen van voedingstoffen aan de cellen. In hoofdstuk 3 heb ik bioluminescente beeldvorming (BLI) geëvalueerd om de cellulaire respons op het omringende zuurstof niveau te onderzoeken. Dit is gedaan in $3 \mathrm{D}$ constructen zowel in vitro als in vivo. Zuurstof is hierbij genomen als molecuul representatief voor de voedingstoffen in de omgeving, en hiervoor is een genfragment van het hypoxia responsive element (HRE) gebruikt als promoter voor het luciferase gen. De luciferase activiteit kan vastgesteld worden door de licht intensiteit te meten wanneer het substraat luciferin wordt toegevoegd, welke gekwantificeerd kan worden door een enzymatische test of met een CCCD camera. Met dit reporterconstruct werd beperkte zuurstofbeschikbaarheid waargenomen in 3D constructen in statische kweken, maar niet in dynamisch doorstroomde bioreactoren. Voor de eerste keer is dit direct bewijs voor het voordeel van bioreactoren boven statische kweken. Bovendien werd in een nagebootst 3D implantaat, bestaande uit meerdere gestapelde en met cellen beklede membraanlagen, een gradient van zuurstof diffusie waargenomen. Omdat BLI een niet-invasieve techniek is, heeft dit bovendien het voordeel dat dezelfde samples op verschillende tijdspunten bekeken kunnen worden. Ik heb dus geobserveerd dat cellen een tekort aan zuurstof (of voedingsstoffen) hadden, en dood gingen na subcutane implantatie in naakte muizen. Dit geeft wederom de noodzaak aan van een vasculair netwerk om cellen te laten overleven. Hierin verder gaande, laten we in hoofdstuk 4 een beperkte afstand voor zuurstofdiffusie zien tot $100-200 \mu \mathrm{m}$ vanaf de bron in HRE-groen fluorescente cellen (GFP) in 3D gel constructen.

Verder wordt de moleculaire diffusie in hydrogelen bestudeerd in hoofdstuk 4 met zowel BLI van promoter-luciferase cellen als met fluorescentie herstel na photobleaching (FRAP) van fluorescente moleculen. Hierbij werd gevonden dat moleculaire diffusie afhankelijk is van zowel de moleculaire grootte als de gelconcentratie. Optische beeldvormingin weefselregeneratie is dus meer dan het enkel het in beeld brengen van cellen of differentiatie. FRAP zou directe informatie kunnen geven over diffusie van voedingstoffen in 
biomaterialen. In combinatie met cellulaire testen, zou dit ook een heel geschikte methode kunnen zijn om eigenschappen van biomaterialen te evalueren en zo het ontwerp van scaffolds te optimaliseren om de aanvoer van voedingstoffen en groeifactoren aan cellen te verbeteren.

We hebben BLI ook gebruikt voor de derde stap in bot weefselregeneratie; in vivo diermodellen, waar het luciferasegen onder controle was van promoters van botspecifieke markers. Met botspecifiek collageen I als promoter, zond de Col(I)-Luc muizen lijn sterk licht uit in botweefsels. Herstel van bot als gevolg van een breuk of verwonding leidde tot sterk licht vanuit de wond. Na implantatie van rhBMP2 in vivo, kon de ectopische botformatie gevolgd worden door de lichtintensiteit te volgen. Omdat dezelfde muis als interne controle kan worden gebruikt geeft dit BLI nog een voordeel: er zijn minder dieren nodig voor statistieken. Deze muislijn heeft dus veel potentieel, omdat het gebruikt kan worden om bot remodeling processen te bestuderen, net als de osteoinductieve moleculen en de scaffolds.

Tot slot worden in hoofdstuk 6 de resultaten van dit proefschrift samengevat en besproken en worden er een aantal ideeën voor toekomstig werk toegelicht. Samengevat, ik heb hier succesvol laten zien dat bioluminescente beeldvorming gebruikt kan worden als toepassing in bot weefsel regeneratie op zowel cellulair als dierlijk niveau. 


\section{REFERENCE}

1. Klerk CP, Overmeer RM, Niers TM, Versteeg HH, Richel DJ, Buckle T, et al. Validity of bioluminescence measurements for noninvasive in vivo imaging of tumor load in small animals. Biotechniques 2007 Jul;43(1 Suppl):7-13, 30.

2. Langer R, Vacanti JP. Tissue engineering. Science 1993 May 14;260(5110):920-926.

3. Muschler GF, Nakamoto C, Griffith LG. Engineering principles of clinical cell-based tissue engineering. J Bone Joint Surg Am 2004 Jul;86-A(7):1541-1558.

4. Parikh SN. Bone graft substitutes: past, present, future. J Postgrad Med 2002 Apr-Jun;48(2):142-148.

5. Friedenstein AJ, Latzinik NW, Grosheva AG, Gorskaya UF. Marrow microenvironment transfer by heterotopic transplantation of freshly isolated and cultured cells in porous sponges. Exp Hematol 1982 Feb;10(2):217-227.

6. Caplan AI. Mesenchymal stem cells. J Orthop Res 1991 Sep;9(5):641-650.

7. Goshima J, Goldberg VM, Caplan AI. The origin of bone formed in composite grafts of porous calcium phosphate ceramic loaded with marrow cells. Clin Orthop Relat Res $1991 \operatorname{Aug}(269): 274-283$.

8. Warnke PH, Springer IN, Wiltfang J, Acil Y, Eufinger H, Wehmoller M, et al. Growth and transplantation of a custom vascularised bone graft in a man. Lancet 2004 Aug 28-Sep 3;364(9436):766-770.

9. Meijer GJ, de Bruijn JD, Koole R, van Blitterswijk CA. Cell based bone tissue engineering in jaw defects. Biomaterials 2008 Jul;29(21):3053-3061.

10. Derubeis AR, Cancedda R. Bone marrow stromal cells (BMSCs) in bone engineering: limitations and recent advances. Ann Biomed Eng 2004 Jan;32(1):160-165. 11. Meijer GJ, de Bruijn JD, Koole R, van Blitterswijk CA. Cell-based bone tissue engineering. PLoS Med 2007 Feb;4(2):e9.

12. Friedenstein A. Stromal-hematopoietic interrelationships: Maximov's ideas and modern models. Haematol Blood Transfus 1989;32:159-167.

13. Friedenstein AJ, Chailakhyan RK, Latsinik NV, Panasyuk AF, Keiliss-Borok IV. Stromal cells responsible for transferring the microenvironment of the hemopoietic tissues. Cloning in vitro and retransplantation in vivo. Transplantation 1974 Apr;17(4):331-340.

14. Bellows CG, Aubin JE. Determination of numbers of osteoprogenitors present in isolated fetal rat calvaria cells in vitro. Dev Biol 1989 May;133(1):8-13.

15. Friedenstein AJ, Chailakhjan RK, Lalykina KS. The development of fibroblast colonies in monolayer cultures of guinea-pig bone marrow and spleen cells. Cell Tissue Kinet 1970 Oct;3(4):393-403. 
16. Pittenger MF, Mackay AM, Beck SC, Jaiswal RK, Douglas R, Mosca JD, et al. Multilineage potential of adult human mesenchymal stem cells. Science 1999 Apr 2;284(5411):143-147.

17. Caplan AI, Bruder SP. Mesenchymal stem cells: building blocks for molecular medicine in the 21st century. Trends Mol Med 2001 Jun;7(6):259-264.

18. Cowan CM, Shi YY, Aalami OO, Chou YF, Mari C, Thomas R, et al. Adipose-derived adult stromal cells heal critical-size mouse calvarial defects. Nat Biotechnol 2004 May;22(5):560-567.

19. Oreffo RO, Bennett A, Carr AJ, Triffitt JT. Patients with primary osteoarthritis show no change with ageing in the number of osteogenic precursors. Scand $\mathrm{J}$ Rheumatol 1998;27(6):415-424.

20. Miao Z, Jin J, Chen L, Zhu J, Huang W, Zhao J, et al. Isolation of mesenchymal stem cells from human placenta: comparison with human bone marrow mesenchymal stem cells. Cell Biol Int 2006 Sep;30(9):681-687.

21. Gronthos S, Graves SE, Ohta S, Simmons PJ. The STRO-1+ fraction of adult human bone marrow contains the osteogenic precursors. Blood 1994 Dec 15;84(12):4164-4173.

22. Jiang Y, Jahagirdar BN, Reinhardt RL, Schwartz RE, Keene CD, OrtizGonzalez XR, et al. Pluripotency of mesenchymal stem cells derived from adult marrow. Nature 2002 Jul 4;418(6893):41-49.

23. Siddappa R, Licht R, van Blitterswijk C, de Boer J. Donor variation and loss of multipotency during in vitro expansion of human mesenchymal stem cells for bone tissue engineering. J Orthop Res 2007 Aug;25(8):1029-1041.

24. Kang YJ, Jeon ES, Song HY, Woo JS, Jung JS, Kim YK, et al. Role of c-Jun $\mathrm{N}$-terminal kinase in the PDGF-induced proliferation and migration of human adipose tissue-derived mesenchymal stem cells. J Cell Biochem 2005 Aug 15;95(6):1135-1145.

25. Tamama K, Fan VH, Griffith LG, Blair HC, Wells A. Epidermal growth factor as a candidate for ex vivo expansion of bone marrow-derived mesenchymal stem cells. Stem Cells 2006 Mar;24(3):686-695.

26. Sotiropoulou PA, Perez SA, Salagianni M, Baxevanis CN, Papamichail M. Characterization of the optimal culture conditions for clinical scale production of human mesenchymal stem cells. Stem Cells 2006 Feb;24(2):462-471.

27. de Boer J, Siddappa R, Gaspar C, van Apeldoorn A, Fodde R, van Blitterswijk C. Wnt signaling inhibits osteogenic differentiation of human mesenchymal stem cells. Bone 2004 May;34(5):818-826.

28. Siddappa R, Martens A, Doorn J, Leusink A, Olivo C, Licht R, et al. cAMP/PKA pathway activation in human mesenchymal stem cells in vitro results in robust bone formation in vivo. Proc Natl Acad Sci U S A 2008 May 20;105(20):72817286. 
29. Boland GM, Perkins G, Hall DJ, Tuan RS. Wnt 3a promotes proliferation and suppresses osteogenic differentiation of adult human mesenchymal stem cells. J Cell Biochem 2004 Dec 15;93(6):1210-1230.

30. Ducy P, Zhang R, Geoffroy V, Ridall AL, Karsenty G. Osf2/Cbfa1: a transcriptional activator of osteoblast differentiation. Cell 1997 May 30;89(5):747-754.

31. Ducy P. Cbfa1: a molecular switch in osteoblast biology. Dev Dyn 2000 Dec;219(4):461-471.

32. Lecanda F, Warlow PM, Sheikh S, Furlan F, Steinberg TH, Civitelli R. Connexin43 deficiency causes delayed ossification, craniofacial abnormalities, and osteoblast dysfunction. J Cell Biol 2000 Nov 13;151(4):931-944.

33. McBeath R, Pirone DM, Nelson CM, Bhadriraju K, Chen CS. Cell shape, cytoskeletal tension, and RhoA regulate stem cell lineage commitment. Dev Cell 2004 Apr;6(4):483-495.

34. Habibovic P, Yuan H, van den Doel M, Sees TM, van Blitterswijk CA, de Groot K. Relevance of osteoinductive biomaterials in critical-sized orthotopic defect. J Orthop Res 2006 May;24(5):867-876.

35. Dalby MJ, Gadegaard N, Tare R, Andar A, Riehle MO, Herzyk P, et al. The control of human mesenchymal cell differentiation using nanoscale symmetry and disorder. Nat Mater 2007 Dec;6(12):997-1003.

36. Folkman J. Tumor angiogenesis: therapeutic implications. N Engl J Med 1971 Nov 18;285(21):1182-1186.

37. Colton CK. Implantable biohybrid artificial organs. Cell Transplant 1995 JulAug;4(4):415-436.

38. Brittberg M, Lindahl A, Nilsson A, Ohlsson C, Isaksson O, Peterson L. Treatment of deep cartilage defects in the knee with autologous chondrocyte transplantation. N Engl J Med 1994 Oct 6;331(14):889-895.

39. Kirsner RS, Falanga V, Eaglstein WH. The development of bioengineered skin. Trends Biotechnol 1998 Jun;16(6):246-249.

40. Shin'oka T, Imai Y, Ikada Y. Transplantation of a tissue-engineered pulmonary artery. N Engl J Med 2001 Feb 15;344(7):532-533.

41. Radisic M, Malda J, Epping E, Geng W, Langer R, Vunjak-Novakovic G. Oxygen gradients correlate with cell density and cell viability in engineered cardiac tissue. Biotechnology and bioengineering 2006 Feb 5;93(2):332-343.

42. Malda J, Rouwkema J, Martens DE, Le Comte EP, Kooy FK, Tramper J, et al. Oxygen gradients in tissue-engineered PEGT/PBT cartilaginous constructs: measurement and modeling. Biotechnology and bioengineering 2004 Apr 5;86(1):9-18.

43. Griffith LG, Naughton G. Tissue engineering--current challenges and expanding opportunities. Science 2002 Feb 8;295(5557):1009-1014.

44. Malda J, Klein TJ, Upton Z. The roles of hypoxia in the in vitro engineering of tissues. Tissue Eng 2007 Sep;13(9):2153-2162. 
45. Lewis MC, Macarthur BD, Malda J, Pettet G, Please CP. Heterogeneous proliferation within engineered cartilaginous tissue: the role of oxygen tension. Biotechnol Bioeng 2005 Sep 5;91(5):607-615.

46. Papadaki M, Bursac N, Langer R, Merok J, Vunjak-Novakovic G, Freed LE. Tissue engineering of functional cardiac muscle: molecular, structural, and electrophysiological studies. Am J Physiol Heart Circ Physiol 2001 Jan;280(1):H168178.

47. Rivron NC, Liu JJ, Rouwkema J, de Boer J, van Blitterswijk CA. Engineering vascularised tissues in vitro. Eur Cell Mater 2008;15:27-40.

48. Druecke D, Langer S, Lamme E, Pieper J, Ugarkovic M, Steinau HU, et al. Neovascularization of poly(ether ester) block-copolymer scaffolds in vivo: long-term investigations using intravital fluorescent microscopy. J Biomed Mater Res A 2004 Jan 1;68(1):10-18.

49. Martin I, Wendt $\mathrm{D}$, Heberer M. The role of bioreactors in tissue engineering. Trends Biotechnol 2004 Feb;22(2):80-86.

50. Kruyt MC, de Bruijn JD, Yuan H, van Blitterswijk CA, Verbout AJ, Oner FC, et al. Optimization of bone tissue engineering in goats: a peroperative seeding method using cryopreserved cells and localized bone formation in calcium phosphate scaffolds. Transplantation 2004 Feb 15;77(3):359-365.

51. Radisic M, Marsano A, Maidhof R, Wang Y, Vunjak-Novakovic G. Cardiac tissue engineering using perfusion bioreactor systems. Nat Protoc 2008;3(4):719-738.

52. Richardson TP, Peters MC, Ennett AB, Mooney DJ. Polymeric system for dual growth factor delivery. Nat Biotechnol 2001 Nov;19(11):1029-1034.

53. Perets A, Baruch Y, Weisbuch F, Shoshany G, Neufeld G, Cohen S. Enhancing the vascularization of three-dimensional porous alginate scaffolds by incorporating controlled release basic fibroblast growth factor microspheres. J Biomed Mater Res A 2003 Jun 15;65(4):489-497.

54. Samee M, Kasugai S, Kondo H, Ohya K, Shimokawa H, Kuroda S. Bone morphogenetic protein-2 (BMP-2) and vascular endothelial growth factor (VEGF) transfection to human periosteal cells enhances osteoblast differentiation and bone formation. J Pharmacol Sci 2008 Sep;108(1):18-31.

55. Levenberg S, Rouwkema J, Macdonald M, Garfein ES, Kohane DS, Darland DC, et al. Engineering vascularized skeletal muscle tissue. Nat Biotechnol 2005 Jul;23(7):879-884.

56. Hung SC, Pochampally RR, Hsu SC, Sanchez C, Chen SC, Spees J, et al. Short-term exposure of multipotent stromal cells to low oxygen increases their expression of CX3CR1 and CXCR4 and their engraftment in vivo. PLoS ONE 2007;2(5):e416.

57. Levy AP, Levy NS, Goldberg MA. Post-transcriptional regulation of vascular endothelial growth factor by hypoxia. J Biol Chem 1996 Feb 2;271(5):2746-2753. 
58. Shima DT, Deutsch U, D'Amore PA. Hypoxic induction of vascular endothelial growth factor (VEGF) in human epithelial cells is mediated by increases in mRNA stability. FEBS Lett 1995 Aug 21;370(3):203-208.

59. Stein I, Neeman M, Shweiki D, Itin A, Keshet E. Stabilization of vascular endothelial growth factor mRNA by hypoxia and hypoglycemia and coregulation with other ischemia-induced genes. Mol Cell Biol 1995 Oct;15(10):5363-5368.

60. Jukes JM, Both SK, Leusink A, Sterk LM, van Blitterswijk CA, de Boer J. Endochondral bone tissue engineering using embryonic stem cells. Proc Natl Acad Sci U S A 2008 May 13;105(19):6840-6845.

61. Budinger TF, Benaron DA, Koretsky AP. Imaging transgenic animals. Annu Rev Biomed Eng 1999;1:611-648.

62. Mayer-Kuckuk P, Boskey AL. Molecular imaging promotes progress in orthopedic research. Bone 2006 Nov;39(5):965-977.

63. Hartman EH, Pikkemaat JA, Vehof JW, Heerschap A, Jansen JA, Spauwen PH. In vivo magnetic resonance imaging explorative study of ectopic bone formation in the rat. Tissue Eng 2002 Dec;8(6):1029-1036.

64. Gasser JA, Ingold P, Grosios K, Laib A, Hammerle S, Koller B. Noninvasive monitoring of changes in structural cancellous bone parameters with a novel prototype micro-CT. J Bone Miner Metab 2005;23 Suppl:90-96.

65. Installe J, Nzeusseu A, Bol A, Depresseux G, Devogelaer JP, Lonneux M. (18)F-fluoride PET for monitoring therapeutic response in Paget's disease of bone. J Nucl Med 2005 Oct;46(10):1650-1658.

66. Even-Sapir E, Metser U, Mishani E, Lievshitz G, Lerman H, Leibovitch I. The detection of bone metastases in patients with high-risk prostate cancer: 99mTc-MDP Planar bone scintigraphy, single- and multi-field-of-view SPECT, 18F-fluoride PET, and 18F-fluoride PET/CT. J Nucl Med 2006 Feb;47(2):287-297.

67. Mahmood S, Martinez de Llano SR. Paget disease of the humerus mimicking metastatic disease in a patient with metastatic malignant mesothelioma on whole body F-18 FDG PET/CT. Clin Nucl Med 2008 Jul;33(7):510-512.

68. Contag $\mathrm{CH}$, Bachmann $\mathrm{MH}$. Advances in in vivo bioluminescence imaging of gene expression. Annu Rev Biomed Eng 2002;4:235-260.

69. Ntziachristos V, Ripoll J, Wang LV, Weissleder R. Looking and listening to light: the evolution of whole-body photonic imaging. Nat Biotechnol 2005 Mar;23(3):313-320.

70. Massoud TF, Gambhir SS. Molecular imaging in living subjects: seeing fundamental biological processes in a new light. Genes Dev 2003 Mar 1;17(5):545-580. 71. Koo V, Hamilton PW, Williamson K. Non-invasive in vivo imaging in small animal research. Cell Oncol 2006;28(4):127-139. 
72. Bogdanovic Z, Bedalov A, Krebsbach PH, Pavlin D, Woody CO, Clark SH, et al. Upstream regulatory elements necessary for expression of the rat COL1A1 promoter in transgenic mice. J Bone Miner Res 1994 Feb;9(2):285-292.

73. Kalajzic I, Kalajzic Z, Kaliterna M, Gronowicz G, Clark SH, Lichtler AC, et al. Use of type I collagen green fluorescent protein transgenes to identify subpopulations of cells at different stages of the osteoblast lineage. J Bone Miner Res 2002 Jan;17(1):1525 .

74. Boban I, Jacquin C, Prior K, Barisic-Dujmovic T, Maye P, Clark SH, et al. The $3.6 \mathrm{~kb}$ DNA fragment from the rat Collal gene promoter drives the expression of genes in both osteoblast and osteoclast lineage cells. Bone 2006 Dec;39(6):1302-1312.

75. Braut A, Kalajzic I, Kalajzic Z, Rowe DW, Kollar EJ, Mina M. Colla1-GFP transgene expression in developing incisors. Connect Tissue Res 2002;43(2-3):216-219.

76. Herschman HR. Molecular imaging: looking at problems, seeing solutions. Science 2003 Oct 24;302(5645):605-608.

77. de Boer J, van Blitterswijk C, Lowik C. Bioluminescent imaging: emerging technology for non-invasive imaging of bone tissue engineering. Biomaterials 2006 Mar;27(9):1851-1858.

78. Maggi A, Ottobrini L, Biserni A, Lucignani G, Ciana P. Techniques: reporter mice - a new way to look at drug action. Trends Pharmacol Sci 2004 Jun;25(6):337-342. 79. Luker GD, Luker KE. Optical imaging: current applications and future directions. J Nucl Med 2008 Jan;49(1):1-4.

80. Harada H, Kizaka-Kondoh S, Itasaka S, Shibuya K, Morinibu A, Shinomiya K, et al. The combination of hypoxia-response enhancers and an oxygen-dependent proteolytic motif enables real-time imaging of absolute HIF-1 activity in tumor xenografts. Biochem Biophys Res Commun 2007 Sep 7;360(4):791-796.

81. Blum JS, Temenoff JS, Park H, Jansen JA, Mikos AG, Barry MA. Development and characterization of enhanced green fluorescent protein and luciferase expressing cell line for non-destructive evaluation of tissue engineering constructs. Biomaterials 2004 Dec;25(27):5809-5819.

82. Olivo C, Alblas J, Verweij V, Van Zonneveld AJ, Dhert WJ, Martens AC. In vivo bioluminescence imaging study to monitor ectopic bone formation by luciferase gene marked mesenchymal stem cells. J Orthop Res 2008 Jul;26(7):901-909.

83. Kumar S, Mahendra G, Ponnazhagan S. Determination of osteoprogenitorspecific promoter activity in mouse mesenchymal stem cells by recombinant adenoassociated virus transduction. Biochim Biophys Acta 2005 Nov 10;1731(2):95-103.

84. Monteiro RM, de Sousa Lopes SM, Korchynskyi O, ten Dijke P, Mummery CL. Spatio-temporal activation of Smad1 and Smad5 in vivo: monitoring transcriptional activity of Smad proteins. J Cell Sci 2004 Sep 15;117(Pt 20):4653-4663. 
85. Zhang N, Fang Z, Contag PR, Purchio AF, West DB. Tracking angiogenesis induced by skin wounding and contact hypersensitivity using a Vegfr2-luciferase transgenic mouse. Blood 2004 Jan 15;103(2):617-626.

86. Iris B, Zilberman Y, Zeira E, Galun E, Honigman A, Turgeman G, et al. Molecular imaging of the skeleton: quantitative real-time bioluminescence monitoring gene expression in bone repair and development. J Bone Miner Res 2003 Mar;18(3):570-578.

87. Gafni Y, Pelled G, Zilberman Y, Turgeman G, Apparailly F, Yotvat H, et al. Gene therapy platform for bone regeneration using an exogenously regulated, AAV-2based gene expression system. Mol Ther 2004 Apr;9(4):587-595.

88. Carlsen H, Moskaug JO, Fromm SH, Blomhoff R. In vivo imaging of NFkappa B activity. J Immunol 2002 Feb 1;168(3):1441-1446.

89. Zhang N, Weber A, Li B, Lyons R, Contag PR, Purchio AF, et al. An inducible nitric oxide synthase-luciferase reporter system for in vivo testing of anti-inflammatory compounds in transgenic mice. J Immunol 2003 Jun 15;170(12):6307-6319.

90. Zhang N, Ahsan MH, Purchio AF, West DB. Serum amyloid A-luciferase transgenic mice: response to sepsis, acute arthritis, and contact hypersensitivity and the effects of proteasome inhibition. J Immunol 2005 Jun 15;174(12):8125-8134.

91. Cowey S, Szafran AA, Kappes J, Zinn KR, Siegal GP, Desmond RA, et al. Breast cancer metastasis to bone: evaluation of bioluminescent imaging and microSPECT/CT for detecting bone metastasis in immunodeficient mice. Clin Exp Metastasis 2007;24(5):389-401.

92. Wetterwald A, van der Pluijm G, Que I, Sijmons B, Buijs J, Karperien M, et al. Optical imaging of cancer metastasis to bone marrow: a mouse model of minimal residual disease. Am J Pathol 2002 Mar;160(3):1143-1153.

93. Zhang J, Lu Y, Dai J, Yao Z, Kitazawa R, Kitazawa S, et al. In vivo real-time imaging of TGF-beta-induced transcriptional activation of the RANK ligand gene promoter in intraosseous prostate cancer. Prostate 2004 Jun 1;59(4):360-369.

94. Zhang W, Feng JQ, Harris SE, Contag PR, Stevenson DK, Contag CH. Rapid in vivo functional analysis of transgenes in mice using whole body imaging of luciferase expression. Transgenic Res 2001 Oct;10(5):423-434.

95. Gopalakrishnan R, Thomas PE, Benson MD, Wang D, Franceschi RT. A homeodomain protein binding element in the bone sialoprotein promoter is critical for tissue-specific expression in bone. Connect Tissue Res 2003;44 Suppl 1:154-160.

96. Opas EE, Gentile MA, Rossert JA, de Crombrugghe B, Rodan GA, Schmidt A. Parathyroid hormone and prostaglandin E2 preferentially increase luciferase levels in bone of mice harboring a luciferase transgene controlled by elements of the proalpha1(I) collagen promoter. Bone 2000 Jan;26(1):27-32.

97. Clemens TL, Tang H, Maeda S, Kesterson RA, Demayo F, Pike JW, et al. Analysis of osteocalcin expression in transgenic mice reveals a species difference in 
vitamin D regulation of mouse and human osteocalcin genes. J Bone Miner Res 1997 Oct;12(10):1570-1576.

98. Lampasso JD, Marzec N, Margarone J, 3rd, Dziak R. Role of protein kinase C alpha in primary human osteoblast proliferation. J Bone Miner Res 2002 Nov;17(11):1968-1976.

99. Kim HJ, Kim JH, Bae SC, Choi JY, Ryoo HM. The protein kinase C pathway plays a central role in the fibroblast growth factor-stimulated expression and transactivation activity of Runx2. J Biol Chem 2003 Jan 3;278(1):319-326.

100. Tu X, Joeng KS, Nakayama KI, Nakayama K, Rajagopal J, Carroll TJ, et al. Noncanonical Wnt signaling through $\mathrm{G}$ protein-linked PKCdelta activation promotes bone formation. Dev Cell 2007 Jan;12(1):113-127.

101. JE Eyckmans AB, FP Luyten. Mode of Action of Ectopic Bone Formation induced by Periosteum-Derived Progenitor Cells. 2006.

102. Dezawa M, Ishikawa H, Itokazu Y, Yoshihara T, Hoshino M, Takeda S, et al. Bone marrow stromal cells generate muscle cells and repair muscle degeneration. Science 2005 Jul 8;309(5732):314-317.

103. de Bruijn JD, van den Brink I, Mendes S, Dekker R, Bovell YP, van Blitterswijk CA. Bone induction by implants coated with cultured osteogenic bone marrow cells. Adv Dent Res 1999 Jun;13:74-81.

104. Haynesworth SE, Goshima J, Goldberg VM, Caplan AI. Characterization of cells with osteogenic potential from human marrow. Bone 1992;13(1):81-88.

105. de Boer J, Licht R, Bongers M, van der Klundert T, Arends R, van Blitterswijk C. Inhibition of histone acetylation as a tool in bone tissue engineering. Tissue Eng 2006 Oct;12(10):2927-2937.

106. Siddappa R, Fernandes H, Liu J, van Blitterswijk C, de Boer J. The response of human mesenchymal stem cells to osteogenic signals and its impact on bone tissue engineering. Curr Stem Cell Res Ther 2007 Sep;2(3):209-220.

107. Newton AC. Regulation of protein kinase C. Curr Opin Cell Biol 1997 Apr;9(2):161-167.

108. Keranen LM, Dutil EM, Newton AC. Protein kinase C is regulated in vivo by three functionally distinct phosphorylations. Curr Biol 1995 Dec 1;5(12):1394-1403.

109. Mellor H, Parker PJ. The extended protein kinase C superfamily. Biochem J 1998 Jun 1;332 ( Pt 2):281-292.

110. Newton AC. Regulation of the ABC kinases by phosphorylation: protein kinase C as a paradigm. Biochem J 2003 Mar 1;370(Pt 2):361-371.

111. Lint JV, Rykx A, Vantus T, Vandenheede JR. Getting to know protein kinase D. Int J Biochem Cell Biol 2002 Jun;34(6):577-581.

112. Sanders JL, Stern PH. Expression and phorbol ester-induced down-regulation of protein kinase C isozymes in osteoblasts. J Bone Miner Res 1996 Dec;11(12):18621872 . 
113. Wang $\mathrm{C}$, Steer JH, Joyce DA, Yip KH, Zheng MH, Xu J. 12-Otetradecanoylphorbol-13-acetate (TPA) inhibits osteoclastogenesis by suppressing RANKL-induced NF-kappaB activation. J Bone Miner Res 2003 Dec;18(12):2159-2168. 114. Radeff JM, Nagy Z, Stern PH. Involvement of PKC-beta in PTH, TNF-alpha, and IL-1 beta effects on IL-6 promoter in osteoblastic cells and on PTH-stimulated bone resorption. Exp Cell Res 2001 Aug 15;268(2):179-188.

115. Yang X, Halladay D, Onyia JE, Martin TJ, Chandrasekhar S. Protein Kinase C is a mediator of the synthesis and secretion of osteoprotegerin in osteoblast-like cells. Biochem Biophys Res Commun 2002 Jan 11;290(1):42-46.

116. Rougier F, Cornu E, Praloran V, Denizot Y. IL-6 and IL-8 production by human bone marrow stromal cells. Cytokine $1998 \mathrm{Feb} ; 10(2): 93-97$.

117. Gliki G, Wheeler-Jones C, Zachary I. Vascular endothelial growth factor induces protein kinase $\mathrm{C}$ (PKC)-dependent $\mathrm{Akt} / \mathrm{PKB}$ activation and phosphatidylinositol 3'-kinase-mediates PKC delta phosphorylation: role of PKC in angiogenesis. Cell Biol Int 2002;26(9):751-759.

118. Esbrit P, Alvarez-Arroyo MV, De Miguel F, Martin O, Martinez ME, Caramelo C. C-terminal parathyroid hormone-related protein increases vascular endothelial growth factor in human osteoblastic cells. J Am Soc Nephrol 2000 Jun;11(6):1085-1092.

119. Swarthout JT, D'Alonzo RC, Selvamurugan N, Partridge NC. Parathyroid hormone-dependent signaling pathways regulating genes in bone cells. Gene 2002 Jan 9;282(1-2):1-17.

120. Yang D, Singh R, Divieti P, Guo J, Bouxsein ML, Bringhurst FR. Contributions of parathyroid hormone (PTH)/PTH-related peptide receptor signaling pathways to the anabolic effect of PTH on bone. Bone 2007 Jun;40(6):1453-1461.

121. Somjen D, Zor U, Kaye AM, Harell A, Binderman I. Parathyroid hormone induction of creatine kinase activity and DNA synthesis is mimicked by phospholipase C, diacylglycerol and phorbol ester. Biochim Biophys Acta 1987 Nov 12;931(2):215223.

122. Siddhanti SR, Hartle JE, 2nd, Quarles LD. Forskolin inhibits protein kinase Cinduced mitogen activated protein kinase activity in MC3T3-E1 osteoblasts. Endocrinology 1995 Nov;136(11):4834-4841.

123. Ghayor C, Rey A, Caverzasio J. Prostaglandin-dependent activation of ERK mediates cell proliferation induced by transforming growth factor beta in mouse osteoblastic cells. Bone 2005 Jan;36(1):93-100.

124. Sabatini M, Lesur C, Pacherie M, Pastoureau P, Kucharczyk N, Fauchere JL, et al. Effects of parathyroid hormone and agonists of the adenylyl cyclase and protein kinase C pathways on bone cell proliferation. Bone 1996 Jan;18(1):59-65.

125. Swarthout JT, Doggett TA, Lemker JL, Partridge NC. Stimulation of extracellular signal-regulated kinases and proliferation in rat osteoblastic cells by 
parathyroid hormone is protein kinase C-dependent. J Biol Chem 2001 Mar 9;276(10):7586-7592.

126. Miao D, Tong XK, Chan GK, Panda D, McPherson PS, Goltzman D. Parathyroid hormone-related peptide stimulates osteogenic cell proliferation through protein kinase $\mathrm{C}$ activation of the Ras/mitogen-activated protein kinase signaling pathway. J Biol Chem 2001 Aug 24;276(34):32204-32213.

127. Baylink TM, Mohan S, Fitzsimmons RJ, Baylink DJ. Evaluation of signal transduction mechanisms for the mitogenic effects of prostaglandin E2 in normal human bone cells in vitro. J Bone Miner Res 1996 Oct;11(10):1413-1418.

128. Chan GK, Miao D, Deckelbaum R, Bolivar I, Karaplis A, Goltzman D. Parathyroid hormone-related peptide interacts with bone morphogenetic protein 2 to increase osteoblastogenesis and decrease adipogenesis in pluripotent C3H10T 1/2 mesenchymal cells. Endocrinology 2003 Dec;144(12):5511-5520.

129. Tang CH, Yang RS, Huang TH, Liu SH, Fu WM. Enhancement of fibronectin fibrillogenesis and bone formation by basic fibroblast growth factor via protein kinase C-dependent pathway in rat osteoblasts. Mol Pharmacol 2004 Sep;66(3):440-449.

130. Tang $\mathrm{CH}$, Yang $\mathrm{RS}$, Fu WM. Prostaglandin E2 stimulates fibronectin expression through EP1 receptor, phospholipase C, protein kinase Calpha, and c-Src pathway in primary cultured rat osteoblasts. J Biol Chem 2005 Jun 17;280(24):2290722916.

131. Delannoy P, Lemonnier J, Hay E, Modrowski D, Marie PJ. Protein kinase Cdependent upregulation of $\mathrm{N}$-cadherin expression by phorbol ester in human calvaria osteoblasts. Exp Cell Res 2001 Sep 10;269(1):154-161.

132. Richardson JA, Amantea CM, Kianmahd B, Tetradis S, Lieberman JR, Hahn $\mathrm{TJ}$, et al. Oxysterol-induced osteoblastic differentiation of pluripotent mesenchymal cells is mediated through a PKC- and PKA-dependent pathway. J Cell Biochem 2007 Apr 1;100(5):1131-1145.

133. Both SK, van der Muijsenberg AJ, van Blitterswijk CA, de Boer J, de Bruijn JD. A rapid and efficient method for expansion of human mesenchymal stem cells. Tissue Eng 2007 Jan;13(1):3-9.

134. Weng L, Dai H, Zhan Y, He Y, Stepaniants SB, Bassett DE. Rosetta error model for gene expression analysis. Bioinformatics 2006 May 1;22(9):1111-1121.

135. Livak KJ, Schmittgen TD. Analysis of relative gene expression data using realtime quantitative PCR and the 2(-Delta Delta C(T)) Method. Methods 2001 Dec;25(4):402-408.

136. Nishizuka Y. Intracellular signaling by hydrolysis of phospholipids and activation of protein kinase C. Science 1992 Oct 23;258(5082):607-614.

137. Geng WD, Boskovic G, Fultz ME, Li C, Niles RM, Ohno S, et al. Regulation of expression and activity of four PKC isozymes in confluent and mechanically stimulated UMR-108 osteoblastic cells. J Cell Physiol 2001 Nov;189(2):216-228. 
138. Keenan C, Kelleher D. Protein kinase C and the cytoskeleton. Cell Signal 1998 Apr;10(4):225-232.

139. Cardell M, Landsend AS, Eidet J, Wieloch T, Blackstad TW, Ottersen OP. High resolution immunogold analysis reveals distinct subcellular compartmentation of protein kinase C gamma and delta in rat Purkinje cells. Neuroscience 1998 Feb;82(3):709-725.

140. Kim BG, Kim HJ, Park HJ, Kim YJ, Yoon WJ, Lee SJ, et al. Runx2 phosphorylation induced by fibroblast growth factor-2/protein kinase $\mathrm{C}$ pathways. Proteomics 2006 Feb;6(4):1166-1174.

141. Valin A, de Miguel F, Garcia-Ocana A, Esbrit P. Parathyroid hormone-related protein (107-139) decreases alkaline phosphatase in osteoblastic osteosarcoma cells UMR 106 by a protein kinase C-dependent pathway. Calcif Tissue Int 1999 Aug;65(2):148-151.

142. Mischak H, Goodnight JA, Kolch W, Martiny-Baron G, Schaechtle C, Kazanietz MG, et al. Overexpression of protein kinase C-delta and -epsilon in NIH 3T3 cells induces opposite effects on growth, morphology, anchorage dependence, and tumorigenicity. J Biol Chem 1993 Mar 25;268(9):6090-6096.

143. Rossi F, McNagny M, Smith G, Frampton J, Graf T. Lineage commitment of transformed haematopoietic progenitors is determined by the level of PKC activity. EMBO J 1996 Apr 15;15(8):1894-1901.

144. Cheung WM, Ng WW, Kung AW. Dimethyl sulfoxide as an inducer of differentiation in preosteoblast MC3T3-E1 cells. FEBS Lett 2006 Jan 9;580(1):121-126. 145. Lemonnier J, Ghayor C, Guicheux J, Caverzasio J. Protein kinase Cindependent activation of protein kinase D is involved in BMP-2-induced activation of stress mitogen-activated protein kinases JNK and p38 and osteoblastic cell differentiation. J Biol Chem 2004 Jan 2;279(1):259-264.

146. Celil AB, Campbell PG. BMP-2 and insulin-like growth factor-I mediate Osterix (Osx) expression in human mesenchymal stem cells via the MAPK and protein kinase D signaling pathways. J Biol Chem 2005 Sep 9;280(36):31353-31359.

147. Carmeliet P, Jain RK. Angiogenesis in cancer and other diseases. Nature 2000 Sep 14;407(6801):249-257.

148. Griffith CK, Miller C, Sainson RC, Calvert JW, Jeon NL, Hughes CC, et al. Diffusion limits of an in vitro thick prevascularized tissue. Tissue Eng 2005 JanFeb;11(1-2):257-266.

149. Ishaug-Riley SL, Crane-Kruger GM, Yaszemski MJ, Mikos AG. Threedimensional culture of rat calvarial osteoblasts in porous biodegradable polymers. Biomaterials 1998 Aug;19(15):1405-1412.

150. Malda J, Woodfield TB, van der Vloodt F, Kooy FK, Martens DE, Tramper J, et al. The effect of PEGT/PBT scaffold architecture on oxygen gradients in tissue engineered cartilaginous constructs. Biomaterials 2004 Nov;25(26):5773-5780. 
151. Wendt D, Stroebel S, Jakob M, John GT, Martin I. Uniform tissues engineered by seeding and culturing cells in 3D scaffolds under perfusion at defined oxygen tensions. Biorheology 2006;43(3-4):481-488.

152. Rouwkema J, Rivron NC, van Blitterswijk CA. Vascularization in tissue engineering. Trends Biotechnol 2008 Aug;26(8):434-441.

153. Roda A, Guardigli M, Pasini P, Mirasoli M. Bioluminescence and chemiluminescence in drug screening. Anal Bioanal Chem 2003 Nov;377(5):826-833.

154. Choy G, Choyke P, Libutti SK. Current advances in molecular imaging: noninvasive in vivo bioluminescent and fluorescent optical imaging in cancer research. Mol Imaging 2003 Oct;2(4):303-312.

155. Luker KE, Luker GD. Applications of bioluminescence imaging to antiviral research and therapy: multiple luciferase enzymes and quantitation. Antiviral Res 2008 Jun;78(3):179-187.

156. Harris AL. Hypoxia--a key regulatory factor in tumour growth. Nat Rev Cancer 2002 Jan;2(1):38-47.

157. Shibata T, Giaccia AJ, Brown JM. Development of a hypoxia-responsive vector for tumor-specific gene therapy. Gene Ther 2000 Mar;7(6):493-498.

158. Mogford JE, Roy NK, Cross KJ, Mustoe TA. Use of hypoxia-inducible factor signal transduction pathway to measure $\mathrm{O} 2$ levels and modulate growth factor pathways. Wound Repair Regen 2003 Nov-Dec;11(6):496-503.

159. Harada H, Kizaka-Kondoh S, Hiraoka M. Optical imaging of tumor hypoxia and evaluation of efficacy of a hypoxia-targeting drug in living animals. Mol Imaging 2005 Jul-Sep;4(3):182-193.

160. Chau NM, Rogers P, Aherne W, Carroll V, Collins I, McDonald E, et al. Identification of novel small molecule inhibitors of hypoxia-inducible factor-1 that differentially block hypoxia-inducible factor-1 activity and hypoxia-inducible factor1alpha induction in response to hypoxic stress and growth factors. Cancer Res 2005 Jun 1;65(11):4918-4928.

161. Shibata T, Akiyama N, Noda M, Sasai K, Hiraoka M. Enhancement of gene expression under hypoxic conditions using fragments of the human vascular endothelial growth factor and the erythropoietin genes. Int J Radiat Oncol Biol Phys 1998 Nov 1;42(4):913-916.

162. Yamazaki Y, Egawa K, Nose K, Kunimoto S, Takeuchi T. HIF-1-dependent VEGF reporter gene assay by a stable transformant of CHO cells. Biol Pharm Bull 2003 Apr;26(4):417-420.

163. Janssen FW, Oostra J, Oorschot A, van Blitterswijk CA. A perfusion bioreactor system capable of producing clinically relevant volumes of tissue-engineered bone: in vivo bone formation showing proof of concept. Biomaterials 2006 Jan;27(3):315-323. 
164. Papenburg BJ, Vogelaar L, Bolhuis-Versteeg LA, Lammertink RG, Stamatialis $\mathrm{D}$, Wessling M. One-step fabrication of porous micropatterned scaffolds to control cell behavior. Biomaterials 2007 Apr;28(11):1998-2009.

165. An WG, Kanekal M, Simon MC, Maltepe E, Blagosklonny MV, Neckers LM. Stabilization of wild-type p53 by hypoxia-inducible factor 1alpha. Nature 1998 Mar 26;392(6674):405-408.

166. Kellner K, Liebsch G, Klimant I, Wolfbeis OS, Blunk T, Schulz MB, et al. Determination of oxygen gradients in engineered tissue using a fluorescent sensor. Biotechnol Bioeng 2002 Oct 5;80(1):73-83.

167. Guarino RD, Dike LE, Haq TA, Rowley JA, Pitner JB, Timmins MR. Method for determining oxygen consumption rates of static cultures from microplate measurements of pericellular dissolved oxygen concentration. Biotechnol Bioeng 2004 Jun 30;86(7):775-787.

168. Brown DA, MacLellan WR, Laks H, Dunn JC, Wu BM, Beygui RE. Analysis of oxygen transport in a diffusion-limited model of engineered heart tissue. Biotechnol Bioeng 2007 Jul 1;97(4):962-975.

169. Jiang BH, Semenza GL, Bauer C, Marti HH. Hypoxia-inducible factor 1 levels vary exponentially over a physiologically relevant range of $\mathrm{O} 2$ tension. Am J Physiol 1996 Oct;271(4 Pt 1):C1172-1180.

170. Radisic M, Deen W, Langer R, Vunjak-Novakovic G. Mathematical model of oxygen distribution in engineered cardiac tissue with parallel channel array perfused with culture medium containing oxygen carriers. Am J Physiol Heart Circ Physiol 2005 Mar;288(3):H1278-1289.

171. Khattak SF, Chin KS, Bhatia SR, Roberts SC. Enhancing oxygen tension and cellular function in alginate cell encapsulation devices through the use of perfluorocarbons. Biotechnol Bioeng 2007 Jan 1;96(1):156-166.

172. Inoue Y, Izawa K, Tojo A, Sekine R, Okubo T, Ohtomo K. Light emission requires exposure to the atmosphere in ex vivo bioluminescence imaging. Mol Imaging 2006 Apr-Jun;5(2):53-56.

173. Moriyama EH, Niedre MJ, Jarvi MT, Mocanu JD, Moriyama Y, Subarsky P, et al. The influence of hypoxia on bioluminescence in luciferase-transfected gliosarcoma tumor cells in vitro. Photochem Photobiol Sci 2008 Jun;7(6):675-680.

174. Guaccio A, Borselli C, Oliviero O, Netti PA. Oxygen consumption of chondrocytes in agarose and collagen gels: a comparative analysis. Biomaterials 2008 Apr;29(10):1484-1493.

175. Ellis SJ, Velayutham M, Velan SS, Petersen EF, Zweier JL, Kuppusamy P, et al. EPR oxygen mapping (EPROM) of engineered cartilage grown in a hollow-fiber bioreactor. Magn Reson Med 2001 Oct;46(4):819-826. 
176. Liu J, Barradas A, Fernandes H, Janssen F, Papenburg B, Stamatialis D, et al. In vitro and in vivo bioluminescence imaging of hypoxia in tissue engineered grafts. Tissue Eng Part C Methods 2009 Aug 17.

177. Presley JF, Cole NB, Schroer TA, Hirschberg K, Zaal KJ, Lippincott-Schwartz J. ER-to-Golgi transport visualized in living cells. Nature 1997 Sep 4;389(6646):81-85.

178. van Royen ME, Farla P, Mattern KA, Geverts B, Trapman J, Houtsmuller AB. Fluorescence Recovery After Photobleaching (FRAP) to Study Nuclear Protein Dynamics in Living Cells. Methods Mol Biol 2009;464:363-385.

179. Axelrod D, Koppel DE, Schlessinger J, Elson E, Webb WW. Mobility measurement by analysis of fluorescence photobleaching recovery kinetics. Biophysical journal 1976 Sep;16(9):1055-1069.

180. Lippincott-Schwartz J, Snapp E, Kenworthy A. Studying protein dynamics in living cells. Nature reviews 2001 Jun;2(6):444-456.

181. Giancoli DC. Physics for scientists and engineers. 3rd ed, 2000.

182. Pinte J, Joly C, Ple K, Dole P, Feigenbaum A. Proposal of a set of model polymer additives designed for confocal FRAP diffusion experiments. J Agric Food Chem 2008 Nov 12;56(21):10003-10011.

183. Leddy HA, Guilak F. Site-specific molecular diffusion in articular cartilage measured using fluorescence recovery after photobleaching. Ann Biomed Eng 2003 JulAug;31(7):753-760.

184. Indovina P, Collini M, Chirico G, Santini MT. Three-dimensional cell organization leads to almost immediate HRE activity as demonstrated by molecular imaging of MG-63 spheroids using two-photon excitation microscopy. FEBS Lett 2007 Feb 20;581(4):719-726.

185. Harada H, Xie X, Itasaka S, Zeng L, Zhu Y, Morinibu A, et al. Diameter of tumor blood vessels is a good parameter to estimate HIF-1-active regions in solid tumors. Biochem Biophys Res Commun 2008 Sep 5;373(4):533-538.

186. Weiss M. Challenges and artifacts in quantitative photobleaching experiments. Traffic (Copenhagen, Denmark) 2004 Sep;5(9):662-671.

187. Rossert J, Eberspaecher H, de Crombrugghe B. Separate cis-acting DNA elements of the mouse pro-alpha $1(\mathrm{I})$ collagen promoter direct expression of reporter genes to different type I collagen-producing cells in transgenic mice. J Cell Biol 1995 Jun;129(5):1421-1432.

188. Rossert JA, Chen SS, Eberspaecher H, Smith CN, de Crombrugghe B. Identification of a minimal sequence of the mouse pro-alpha 1(I) collagen promoter that confers high-level osteoblast expression in transgenic mice and that binds a protein selectively present in osteoblasts. Proc Natl Acad Sci U S A 1996 Feb 6;93(3):10271031 .

189. Peister A, Mellad JA, Larson BL, Hall BM, Gibson LF, Prockop DJ. Adult stem cells from bone marrow (MSCs) isolated from different strains of inbred mice vary 
in surface epitopes, rates of proliferation, and differentiation potential. Blood 2004 Mar 1;103(5):1662-1668.

190. Yamamoto M, Fisher JE, Gentile M, Seedor JG, Leu CT, Rodan SB, et al. The integrin ligand echistatin prevents bone loss in ovariectomized mice and rats. Endocrinology 1998 Mar;139(3):1411-1419.

191. Stock SR, Ignatiev KI, Foster SA, Forman LA, Stern PH. MicroCT quantification of in vitro bone resorption of neonatal murine calvaria exposed to IL-1 or PTH. Journal of structural biology 2004 Aug;147(2):185-199.

192. Troy T, Jekic-McMullen D, Sambucetti L, Rice B. Quantitative comparison of the sensitivity of detection of fluorescent and bioluminescent reporters in animal models. Mol Imaging 2004 Jan;3(1):9-23.

193. Paroo Z, Bollinger RA, Braasch DA, Richer E, Corey DR, Antich PP, et al. Validating bioluminescence imaging as a high-throughput, quantitative modality for assessing tumor burden. Mol Imaging 2004 Apr;3(2):117-124.

194. Contag CH, Contag PR, Mullins JI, Spilman SD, Stevenson DK, Benaron DA. Photonic detection of bacterial pathogens in living hosts. Mol Microbiol 1995 Nov;18(4):593-603.

195. Li X, Quigg RJ, Zhou J, Ryaby JT, Wang H. Early signals for fracture healing. J Cell Biochem 2005 May 1;95(1):189-205.

196. Rundle CH, Wang H, Yu H, Chadwick RB, Davis EI, Wergedal JE, et al. Microarray analysis of gene expression during the inflammation and endochondral bone formation stages of rat femur fracture repair. Bone 2006 Apr;38(4):521-529.

197. Nakazawa T, Nakajima A, Seki N, Okawa A, Kato M, Moriya H, et al. Gene expression of periostin in the early stage of fracture healing detected by cDNA microarray analysis. J Orthop Res 2004 May;22(3):520-525.

198. Shimizu T, Mehdi R, Yoshimura Y, Yoshikawa H, Nomura S, Miyazono K, et al. Sequential expression of bone morphogenetic protein, tumor necrosis factor, and their receptors in bone-forming reaction after mouse femoral marrow ablation. Bone 1998 Aug;23(2):127-133.

199. Einhorn TA. The cell and molecular biology of fracture healing. Clin Orthop Relat Res 1998 Oct(355 Suppl):S7-21.

200. Wozney JM, Rosen V, Celeste AJ, Mitsock LM, Whitters MJ, Kriz RW, et al. Novel regulators of bone formation: molecular clones and activities. Science 1988 Dec 16;242(4885):1528-1534.

201. Wang EA, Rosen V, D'Alessandro JS, Bauduy M, Cordes P, Harada T, et al. Recombinant human bone morphogenetic protein induces bone formation. Proc Natl Acad Sci U S A 1990 Mar;87(6):2220-2224.

202. Okubo Y, Bessho K, Fujimura K, Konishi Y, Kusumoto K, Ogawa Y, et al. Osteoinduction by recombinant human bone morphogenetic protein-2 at intramuscular, 
intermuscular, subcutaneous and intrafatty sites. Int J Oral Maxillofac Surg 2000 Feb;29(1):62-66.

203. Habibovic P, Sees TM, van den Doel MA, van Blitterswijk CA, de Groot K. Osteoinduction by biomaterials--physicochemical and structural influences. J Biomed Mater Res A 2006 Jun 15;77(4):747-762.

204. Zilberman Y, Kallai I, Gafni Y, Pelled G, Kossodo S, Yared W, et al. Fluorescence molecular tomography enables in vivo visualization and quantification of nonunion fracture repair induced by genetically engineered mesenchymal stem cells. J Orthop Res 2008 Apr;26(4):522-530.

205. Noel D, Caton D, Roche S, Bony C, Lehmann S, Casteilla L, et al. Cell specific differences between human adipose-derived and mesenchymal-stromal cells despite similar differentiation potentials. Exp Cell Res 2008 Apr 15;314(7):1575-1584.

206. De Boer J, Wang HJ, Van Blitterswijk C. Effects of Wnt signaling on proliferation and differentiation of human mesenchymal stem cells. Tissue Eng 2004 Mar-Apr;10(3-4):393-401.

207. Siddappa R, Mulder W, Steeghs I, van de Klundert C, Fernandes H, Liu J, et al. cAMP/PKA Signaling Inhibits Osteogenic Differentiation and Bone Formation in Rodent Models. Tissue Eng Part A 2009 Feb 13.

208. Deshpande RR, Heinzle E. On-line oxygen uptake rate and culture viability measurement of animal cell culture using microplates with integrated oxygen sensors. Biotechnol Lett 2004 May;26(9):763-767.

209. Zhao F, Pathi P, Grayson W, Xing Q, Locke BR, Ma T. Effects of oxygen transport on 3-d human mesenchymal stem cell metabolic activity in perfusion and static cultures: experiments and mathematical model. Biotechnol Prog 2005 JulAug;21(4):1269-1280.

210. Fehrer C, Brunauer R, Laschober G, Unterluggauer H, Reitinger S, Kloss F, et al. Reduced oxygen tension attenuates differentiation capacity of human mesenchymal stem cells and prolongs their lifespan. Aging Cell 2007 Dec;6(6):745-757.

211. Lennon DP, Edmison JM, Caplan AI. Cultivation of rat marrow-derived mesenchymal stem cells in reduced oxygen tension: effects on in vitro and in vivo osteochondrogenesis. J Cell Physiol 2001 Jun;187(3):345-355.

212. Torzilli PA, Arduino JM, Gregory JD, Bansal M. Effect of proteoglycan removal on solute mobility in articular cartilage. J Biomech 1997 Sep;30(9):895-902.

213. Maroudas A. Biophysical chemistry of cartilaginous tissues with special reference to solute and fluid transport. Biorheology 1975 Jun;12(3-4):233-248.

214. Pluen A, Netti PA, Jain RK, Berk DA. Diffusion of macromolecules in agarose gels: comparison of linear and globular configurations. Biophys J 1999 Jul;77(1):542552.

215. Lang I, Scholz M, Peters R. Molecular mobility and nucleocytoplasmic flux in hepatoma cells. J Cell Biol 1986 Apr;102(4):1183-1190. 


\section{Acknowledgements}

This thesis is the result of my research at Department of Tissue Regeneration, University of Twente. The texts before here are all based on my scientific observation, analysis, and research. They are rational and solid data. Now it is the time and here is the space for me to write down some emotional words, which are already kept in my heart for long time, to all the people who provided me support, help and joy.

I would like to thank my supervisors prof. Clemens van Blitterswijk and dr. Jan de Boer for offering me the good opportunity to be a PhD in your group and, especially, leading me into the tissue engineering field that I had longed for. Clemens, you are not only inspiring me on the scientific research, but also teaching me an important lesson: science is not only on the paper. Jan, thank you for your scientific guidance, fruitful discussion and input for my English writing. I still remember what you have said: I take my $\mathrm{PhD}$ students as my scientific children. I would like to watch them from crawling to walking to running. Not a single word could describe my appreciation to you in my scientific career. Dr. Marcel Karperien, thank you for your advice with my experiments.

This thesis cannot be completed without the cooperation with Anton Martens from UMC, Roel Arends, Eugene Someren and Koen Dechering from Schering-Plough, Tom Groothuis and Cees Otto from BPE, UT. Thank you for the discussion on the experiments and manuscripts. Also many thanks to HenkJan Prins and Henk Rozemuller from UMC, Bernke Papenburg and Dimitrios Stamatialis from MTG, UT for their help with experiments. Cristina Olive, although we were housemates for a short term, we had a very good time. I always say I can make the authentic Italian tiramisu.

I reall enjoyed my $\mathrm{PhD}$ life wherever in Bilthoven or in Enschede. In such an open and friendly TR group, I never felt alone and lonely. Roka, Jerome and Lorenzo, without your guys, for sure my life in Utrecht would be much more boring. My special thanks go to the scientific brothers and sisters, Ram, Hugo, Andre, Joyce, Hemant and Ana. It is you who make my life and work in Enschede so cheerful. Hugo and Andre, you are the next, good luck. Joyce, thank you for translating my summary into Dutch. Thank Ana and Janneke to be my paranymphs and for everything you have done. Pamela, I would like to watch and discuss "the project runway" with you again. Karolina and Maciek, 
thank you for the parties and polish dumplings. Sandra and Huang, we had a good time when traveling in Poland.

Thanks to my social colleagues in Enscehde: the TR girls, Anindita, Aliz, Anouk, Emilie, Elle, Ineke, Jacqueline Liliana, Nicole and Charlene; the TR boys: Bin, Ling, Liang, Jeroen, Gustavo, Nicolas, Anand, Chris, Bjorn, Roman and Tim. Thank Ane for helping me with the Photoshop, and Philip, Jorge and all guys who were in Geres. I would also thank the senior PhDs, Sanne, Jojanneke, Doreen, Frank, Jeroen, Jeanine, Fluorence, Aart and Maria Liu. Ruud, thank you for showing me how to do the cellular experiments in labs. Thanks to Viola and Audry for your help and assistance.

My special thanks to my friends Jiaping, Sunkang, Huipin, Ruiqing, Wenxiong, Chen Wan, Chen Lei, Yanling, Frizz, Lily, Xu Qiang, Ji Yun and Ma Weixia. Yanling, you are not only a friend to me, but more like a sister, thank you for everything. Lily and Xuqiang, we know each other since I just came to the Netherlands, I really appreciate our friendship. Ji Yun and Ma weixia, I hope we can spend more time together travelling in the future. I would also like to thank my friends at Enschede, Shui Lingling, Jin mingliang, Zhong Zhicheng, Huang Yanhui, Li Chen, Wu Chien-Ching, Li Xiao, Zhang Zheng and Jin Rong. Because of you, I have a more colorful life. In addition, thanks my friends in China and in other countries.

Lastly, I would express my deepest acknowledgements to my family, baba, mama and jiejie. Because of your endless love and enduring support, I am what I am, not only today but everything I have achieved in my 28 years. I love you. Nan, thank you, without you I could not reach here. 2009 is our tenth year together. I believe there are more tenth years coming to you and me.

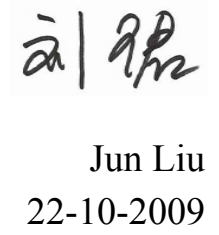




\section{Curriculum Vitae}

Jun Liu was born on January $19^{\text {th }} 1981$ in Linfen, Shanxi Province, China. She obtained her B.Sc (Chemical Engineering) degree at Tianjin University, Tianjin, China in 2002. In 2003, she went to University of Twente, Enschede, The Netherlands, to study Materials Science, for which she received her master

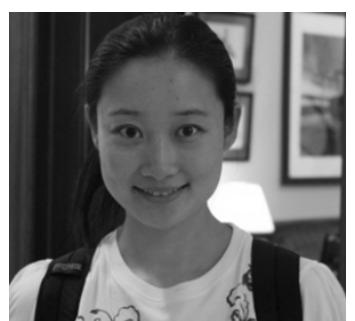
degree with TSP scholarship. In October 2004, she continued her education as a $\mathrm{PhD}$ at Univertsity of Twente under the supervision of Prof. dr. C.A. van Blitterswijk and Dr. J. de Boer. The subject of her research was to apply bioluminescent imaging in bone tissue engineering and the results are described in this thesis. From December 2008, she continues her scientific work at Department of Tissue Regeneration at University of Twente as a research scientist.

\section{List of Publications}

Siddappa R, Fernandes H, Liu J, van Blitterswijk CA, de Boer J. The response of human mesenchymal stem cells to osteogenic signals and its impact on bone tissue engineering. Curr Stem Cell Res Ther. 2007 Sep;2(3):209-20.

Rivron NC, Liu J, Rouwkema J, de Boer J, van Blitterswijk CA. Engineering vascularised tissues in vitro. Eur Cell Mater. 2008 Feb 21;15:27-40.

Siddappa R, Mulder W, Steeghs I, van de Klundert C, Fernandes H, Liu J, Arends R, van Blitterswijk CA, de Boer J. cAMP/PKA signaling inhibits osteogenic differentiation and bone formation in rodent models. Tissue Eng Part A. 2009 Aug;15(8):2135-43.

Papenburg BJ, Liu J, Higuera GA, Barradas AM, de Boer J, van Blitterswijk CA, Wessling M, Stamatialis D. Development and analysis of multi-layer 
scaffolds for tissue engineering. Biomaterials. 2009 Oct;30(31):6228-39. Epub 2009 Aug 11.

Liu J, Barradas A, Fernandes H, Janssen F, Papenburg B, Stamatialis D, Martens AC, van Blitterswijk CA, De Boer J. In vitro and in vivo bioluminescent imaging of hypoxia in tissue engineered grafts. Tissue Eng Part C Methods. 2009 Aug 17. [Epub ahead of print]

Liu J, Someren E, Mentink A, Licht R, Dechering K, van Blitterswijk CA, de Boe J. The effect of PKC activation and inhibition on osteogenic differentiation of human mesenchymal stem cells. Journal of Tissue Engineering and Regenerative Medicine. Resubmitted.

Liu J, Arends R, Martens AC, den Brok-Bardoel MHC, Scheepers MGH, van Blitterswijk CA, de Boer J. Non-invasive imaging of bone specific collagen I expression in a luciferase transgenic mouse model. Tissue Engineering. Resubmitted.

Siddappa R, Doorn J, Liu J, Langerwerf E, Arends R, Blitterswijk CA, de Boer J. Timing rather than the concentration of cyclic AMP correlates to osteogenic differentiation of human mesenchymal stem cells. Journal of Tissue Engineering and Regenerative Medicine. Resubmitted.

Liu J, Hilderink J, Groothuis TAM, Otto C, van Blitterswijk CA, de Boer J. Monitoring nutrient transport in tissue engineered grafts. Submitted.

Teixeira S, Liu J, Petersen W, van Blitterswijk C, Ferraz MP, Monteiro FJ, de Boer J. Evaluation of the scaffolds angiogenic potential using the chick embryo chorioallantoic membrane model - Preliminary studies. In preparation.

\section{Selected Abstracts}

Liu J, van Blitterswijk CA, Martens AC, de Boer J. Non-invasive imaging of hypoxia in tissue engineering. 20th European Conference on Biomaterials (ESB) 2006, Nantes, France. Oral presentation.

Liu J, Licht R, Siddappa R, van Blitterswijk CA, de Boer J. Effects of Protein Kinase $\mathrm{C}$ on Proliferation and Osteogenesis of Human Mesenchymal Stem cells. 
Tissue Engineering and Regenerative Medicine international Society (TERMISEU) 2006. Rotterdam, The Netherlands. Poster presentation.

Liu J, van Blitterswijk CA, de Boer J. Bioluminescent imaging in bone tissue engineering. 15th NVCB conference 2006. Zeist, The Netherlands. Oral presentation.

Liu J, van Blitterswijk CA, de Boer J. Different Protein Kinase C iso-zymes exert opposing effects on Osteogenesis of Human Mesenchymal Stem Cells. 16th NVCB conference 2007. Zeist, The Netherlands. Oral presentation.

Liu J, van Blitterswijk CA, de Boer J. Different Protein Kinase C isozymes exert opposing effects on Osteogenesis of Human Mesenchymal Stem Cells. $16^{\text {th }}$ NBTE conference 2007. Luntern, The Netherlands. Poster presentation.

Liu J, van Blitterswijk CA, de Boer J. Different Protein Kinase C isozymes exert opposing effects on Osteogenesis of Human Mesenchymal Stem Cells. International Bone and Mineral Society, Davos Workshop 2008. Davos, Switzerland. Poster presentation.

Liu J, Barradas A, van Blitterswijk CA, de Boer J. Non-invasive imaging of nutrient availability in tissue engineering. $8^{\text {th }}$ World Biomaterials Congress 2008. Amsterdam, The Netherlands. Oral Presentation.

Liu J, Arends R, Martens AC, van Blitterswijk CA, de Boer J. Non-invasive imaging of collagen type I expression in a transgenic mouse model. TERMISEU 2008. Porto, Portugal. Poster presentation.

Liu J, Arends R, Martens AC, van Blitterswijk CA, de Boer J. Non-invasive imaging of collagen type I expression in a transgenic mouse model. $17^{\text {th }}$ NBTE conference 2008. Luntern, The Netherlands. Oral presentation.

Liu J, Hilderink J, Groothuis TAM, van Blitterswijk CA, de Boer J. Modeling nutrient transport in tissue engineered constructs. $17^{\text {th }}$ NBTE conference 2008. Luntern, The Netherlands. Poster presentation. 
"My own scientific career was a descent from higher to lower dimension, led by a desire to understand life. I went from animals to cells to bacteria, from bacteria to molecules, from molecules to electrons.

The story had its irony, for molecules and electrons have no life at all.

On my way, the life I was trying to study ran out between my fingers."

"The Perils of Reductionism" (1972)

Albert Szent-Gyorgi 